\title{
Lipid Metabolism Gene Expression And Cellularity of Intramuscular Adipocytes Within The Longissimus Muscle of Angus- And Wagyu-Sired Cattle When Raised To A Similar Age or Body Weight
}

Jerad Jaborek

Michigan State College of Agriculture and Applied Science: Michigan State University

Francis Fluharty

University of Georgia College of Agricultural and Environmental Sciences

Kichoon Lee

OSU: The Ohio State University

Henry Zerby

WQSCC

Alejandro Relling ( $\square$ relling.1@osu.edu )

Ohio State University https://orcid.org/0000-0002-1288-3037

\section{Research}

Keywords: age, body weight, cellularity, lipid metabolism, Wagyu

Posted Date: August 5th, 2021

DOI: https://doi.org/10.21203/rs.3.rs-764272/v1

License: (c) (1) This work is licensed under a Creative Commons Attribution 4.0 International License.

Read Full License 


\section{Abstract}

Background: This study investigates intramuscular (IM) adipocyte development and growth in the Longissimus muscle (LM) between Wagyu- and Angus-sired steers compared at a similar age and days on feed (DOF) endpoint or similar body weight (BW) endpoint by measuring IM adipocyte cell area and lipid metabolism gene expression.

Methods: Angus-sired steers (AN, $n=6)$ were compared with steers from two different Wagyu sires, selected for either growth or marbling, to be compared at a similar DOF (WA-GD, $n=5$ and WA-MD, $n=5$ ) in experiment 1 or $B W(W A-G B, n=4$ and $\mathbf{W A - M B}, n=5$ ) in experiment 2 , respectively.

Results: In experiment 1, WA-MD steers had a greater percentage of IM fat in the LM compared with AN and WA-GD steers. In experiment 2, WA-MB steers had a greater percentage of IM fat in the LM compared with AN and WA-GB steers. The distribution of IM adipocyte area was unimodal at all biopsy collections, with IM adipocyte area becoming progressively larger as cattle age and BW increased $(P \leq 0.01)$. Peroxisome proliferator activated receptor delta (PPARd) was upregulated earlier for WA-MD and WA-MB cattle compared with other steers at a similar age and BW ( $P \leq 0.02$; treatment $\times$ biopsy interaction). An earlier upregulation of PPARd is believed to have then upregulated peroxisome proliferator activated receptor gamma (PPARg) at a lesser BW for WA-MB steers $(P=0.09$; treatmentxbiopsy interaction), while WA-MD steers had a greater $(P \leq 0.04)$ overall mean PPARg expression compared with other steers. Glycerol-3-phosphate acyltransferase, lipin 1, and hormone sensitive lipase demonstrated expression patterns similar to PPARg and PPARd or CCAAT enhancer binding protein beta, which emphasizes their importance in marbling development and growth. Additionally, WA-MD and WA-MB steers often had a greater early expression of fatty acid transporters (fatty acid transport protein $1 ; P<0.02$; treatment $\times$ biopsy interaction) and binding proteins (fatty acid binding protein 4) compared with other steers. With many lipolytic genes upregulated at harvest, acetyl-CoA carboxylase beta may be inhibiting fatty acid oxidation in the LM to allow greater IM fat accumulation.

Conclusions: Cattle with a greater marbling propensity appear to upregulate adipogenesis at a lesser maturity through PPARd, PPARg, and possibly adipogenic regulating compounds in lysophosphatidic acid and diacylglycerol.

\section{Background}

Marbling, also known as intramuscular (IM) fat, can be visually recognized as the deposition of fat between the muscle fibers within the muscle bundles of the muscle. Marbling has been reported to have a positive impact on the three sensory characteristics: tenderness, juiciness, and flavor, of beef eating quality and increase consumer acceptability (May et al., 1992; Platter et al., 2003; Killinger et al., 2004). As an indication of eating quality and value, marbling score is extremely influential in determining beef 
carcass USDA quality grade. Therefore, considerable interest has been directed towards expanding the knowledge focused on IM fat development and growth.

Intramuscular adipocytes develop from mesenchymal progenitor cells in a process known as adipogenesis. Adipogenesis begins with the commitment or determination of mesenchymal stem cells to the adipose tissue lineage and formation of fibro/adipogenic progenitor cells, also known as preadipocytes. A cell signaling cascade allows pre-adipocytes to proliferate, followed by pre-adipocytes differentiating into mature adipocytes through the incorporation and storage of triglycerides.

Cattle of varying breed or genetic background can result in significant differences in marbling deposition. For example, Wagyu cattle are known for their ability to deposit extreme amounts of IM fat in Japan (Gotoh et al., 2009; Albrecht et al., 2011), even when compared with the Angus breed that is well recognized for its marbling ability in the United States (Lunt et al., 1993; Lunt et al., 2005). Previous research results from Wang et al. (2009) indicated a critical time period when pre-adipocytes underwent proliferation in cattle at approximately 7 months of age, with differentiation occurring around 12 months of age, followed by increased IM adipocyte hypertrophy.

The objective of the present study was to increase the understanding of the cellular signaling pathways responsible for marbling differences in cattle with different genotypes over time at a similar age endpoint, with a similar number of days consuming a feedlot ration, or at a similar body weight endpoint. Genes analyzed in the present study are representative of adipogenesis, angiogenesis, fatty acid synthesis and transport, triglyceride synthesis and lipolysis signaling pathways. We hypothesized that the expression of genes responsible for marbling accumulation would display different patterns of IM adipogenesis between Angus- and Wagyu-sired steers in relation to marbling deposition. Determining the distribution of IM adipocyte size in combination with measuring the expression of genes associated with different stages of adipogenesis should allow for the identification of the different adipogenic (proliferation and differentiation) stages in cattle.

\section{Methods}

Animal procedures and husbandry practices were approved by the Institutional Animal Care and Use Committee (IACUC; protocol number 2015A00000093) of The Ohio State University and followed the guidelines recommended in the Guide for the Care and Use of Agricultural Animals in Agricultural Research and Teaching (FASS, 2010).

\section{Experiment 1 treatments}

Experiment 1 was designed to compare Angus- and Wagyu-sired steers at a similar age or days on feed (DOF). Angus-sired steers (AN) were sired by GAR Sunrise $(n=6)$. Wagyu-sired steers $(n=5)$ were sired by LMR Fukutsuru 729T (WA-GD), a sire selected for his genetic growth potential. Wagyu-sired steers $(n=5)$ were sired by OW Yasufuku 229Y (WA-MD), a sire selected for his genetic marbling potential. Angus-sired steers were removed from the feedlot for harvest when they reached the targeted body weight (BW) 
endpoint of $613 \mathrm{~kg}$. Wagyu-sired steers from WA-GD and WA-MD were removed for harvest by blocking them with AN steers.

\section{Experiment 2 treatments}

Angus-sired steers from experiment 1 were also used in the comparison with Wagyu-sired cattle at a similar BW endpoint $(613 \mathrm{~kg})$. Wagyu-sired steers $(\mathrm{n}=4)$ sired by LMR Fukutsuru 729T (WA-GB) and Wagyu-sired steers $(n=5)$ sired by OW Yasufuku 229Y (WA-MB) were removed from the feedlot for harvest after reaching $613 \mathrm{~kg}$.

\section{Management, feeding, and biopsy collection}

Steers used in the present study were born from SimAngus cows at the Jackson Agricultural Research Station (JARS; Jackson, OH) in March of 2017. Calves were weighed and weaned at seven months of age [204 $\pm 7.77 \mathrm{~d}$ of age (DOA)]. Calves were backgrounded for $54 \mathrm{~d}$ with free choice hay and a concentrate pellet (consisting of approximately $54 \%$ ground corn, $21 \%$, corn gluten feed, $18 \%$ low-fat dried distillers grain, $3 \%$ soybean meal, $3 \%$ animal-vegetable fat blend, and the remainder consisting of Amaferm (BioZyme, St. Joseph, MO), vitamins, and minerals) before being transported to the Ohio Agricultural Research and Development Center (OARDC; Wooster, $\mathrm{OH}$ ) feedlot. Upon receiving to the feedlot, calves were weighed, ear-tagged, and administered Inforce 3 (Zoetis, Parsippany, NJ) and Vetmetric pour-on (MWI veterinary supply; Northern Ireland). Calves were placed into individual pens $(2.6 \times 1.5 \mathrm{~m})$ consisting of concrete slatted floors, with a $1.5 \mathrm{~m}$ long concrete feed bunk, and ad libitum access to clean, fresh water. No implants were administered to prevent their interference with marbling development and growth.

Diets were formulated to meet the nutrient requirements of growing and finishing beef cattle (NASEM, 2016), with the exception of excluding vitamin A from the supplement. Steers were offered a growing diet for approximately 4 months (119 d) before making a $21 \mathrm{~d}$ transition to the finishing diet, which they consumed until removal for harvest (Table 1). Weaning BW was collected at weaning, receiving BW was collected at the OARDC feedlot upon arrival, and off-test BW was collected at the OARDC feedlot before being transported to the Ohio State University abattoir (Columbus, $\mathrm{OH}$ ). Feedlot performance, carcass data, and fatty acid composition results were reported previously by Jaborek et al. (2020). 
Table 1. Composition (\%) of diets offered during the experiment on a dry matter basis

\section{Growing}

Finishing

Ingredient

Whole shelled corn

17.50

55.00

DDGS

17.50

10.00

Corn silage

55.00

25.00

Supplement

10.00

10.00

Ground corn

1.12

1.51

Soybean meal

5.00

5.00

Digest More ${ }^{1}$

0.78

0.39

Limestone

1.10

1.10

White salt

1.00

1.00

Urea

0.50

0.50

Vit. A, 30,000 IU/g

0.00

0.00

Vit. D, 3,000 IU/g

0.01

0.01

Vit. E, $44 \mathrm{IU} / \mathrm{g}$

0.02

0.02

Selenium, 201 ppm

0.14

0.14

Potassium Chloride

0.30

0.30

Copper Sulfate

0.01

0.01

Zinc Sulfate

0.02

0.02

Magnesium Sulfate

0.01

0.01

Analyzed composition

Crude protein, \%

14.22

12.90

NDF, \%

28.18

17.09

Fat, \%

3.71

3.59

Ca, \%

0.52

0.45

$\mathrm{P}, \%$

0.35

0.33

NEm, Mcal/kg

2.01

2.21 
${ }^{1}$ Amaferm (BioZyme, St. Joesph, MO)

Biopsies were collected at the average age of $205,268,331,422$, and $513 \mathrm{~d}$ for experiment 1 steers. Steers in experiment 2 weighed $241 \pm 18.4,296 \pm 24.3,400 \pm 33.9,531 \pm 33.3$, and $613 \pm 18.0 \mathrm{~kg}$ and were $205 \pm 9.5,280 \pm 10.0,352 \pm 16.3,458 \pm 31.9$, and $546 \pm 50.1$ DOA at the 5 biopsy collections. The longissimus muscle (LM) was biopsied on the left side beginning at the 12th rib. Subsequent biopsies were collected approximately $2.54 \mathrm{~cm}$ posterior from the previous biopsy site, evident by scarring. Prior to surgery, the biopsy site was clipped to remove the hair, scrubbed 3 times with betadine surgical scrub, and followed by 3 alcohol scrubs. Administration of $5 \mathrm{ml}$ of lidocaine was administered prior to making a $2.54 \mathrm{~cm}$ incision to place the biopsy needle. A $10 \mathrm{~mm}$ muscle biopsy cannula (Millennium Surgical Corporation; Narberth, PA) was used to collect approximately $1 \mathrm{~g}$ of LM tissue. The biopsy incision site was stapled and sprayed with a water resistant aerosol bandage (Aluspray; Neogen Corporation; Lexington, $\mathrm{KY}$ ). Biopsies were snap frozen with liquid nitrogen and stored in the freezer at $-80^{\circ} \mathrm{C}$. The harvest (5th / final) LM biopsy was collected from the LM of the carcass immediately after hide removal with a knife and stored in the freezer at $-80^{\circ} \mathrm{C}$.

\section{Cellularity analysis}

Multiple pictures were collected from each histology slide to identify all intramuscular adipocytes using a Thermo Fisher EVOS XL Core Cell Imaging System (Thermo Fisher Scientific, Waltham, MA). The circumference of all traceable IM adipocytes were traced to determine and record each cell's area using ImageJ (NIH, Bethesda, MD). Due to time and financial constraints, slides from the fourth biopsy collection were not traced and analyzed.

\section{RNA extraction and analysis}

Extraction of RNA was performed using RNAzol RT (Molecular Research Center; Cincinnati, OH) according to the manufacturer's instructions. Briefly, $1 \mathrm{ml}$ of RNAzol RT and $0.15 \mathrm{~g}$ of $0.1 \mathrm{~mm}$ zirconium beads were placed into a $2 \mathrm{ml}$ micro-centrifuge tube, along with $0.1 \mathrm{~g}$ of biopsied LM tissue, and chilled on ice. Samples were homogenized for $1 \mathrm{~min}$ with a bead beater, chilled on ice for $1 \mathrm{~min}$, and repeated. RNasefree water $(0.4 \mathrm{ml})$ was added, samples were vortexed for $15 \mathrm{~s}$, and allowed to incubate for $15 \mathrm{~min}$ at room temperature. Next, samples were centrifuged at $12,000 \times \mathrm{g}$ for $5 \mathrm{~min}$ at $4^{\circ} \mathrm{C}$. After, $0.6 \mathrm{ml}$ of supernatant was pipetted into 2 new micro-centrifuge tubes each, containing $0.4 \mathrm{ml}$ of isopropanol, vortexed for $2 \mathrm{~s}$, and allowed to incubate for $10 \mathrm{~min}$ at room temperature, before centrifuging at 12,000 $\times$ $\mathrm{g}$ for $10 \mathrm{~min}$ at $4^{\circ} \mathrm{C}$. Supernatant was discarded; $0.4 \mathrm{ml}$ of $75 \%$ ethanol was pipetted into each tube, and centrifuged at $4,000 \times \mathrm{g}$ for $3 \mathrm{~min}$ at $20^{\circ} \mathrm{C}$. After repeating the last step, the RNA pellet was allowed to dry and $10 \mu \mathrm{l}$ of RNase-free water was pipetted into each tube to re-suspend the RNA pellet. The 2 centrifuge tubes containing RNA were combined and frozen at $-80^{\circ} \mathrm{C}$ until analysis.

Extracted RNA was quantified using UV spectroscopy (NanoDrop Technologies; Wilmington, DE) and RNA integrity was assessed using a BioAnalyzer 2100 and RNA NanoChip assay (Agilent Technologies; Santa 
Clara, CA). Gene expression was determined using a NanoString nCounter XT Assay (NanoString Technologies; Seattle, WA). The nSolver Analysis Software 3.0 (NanoString Technologies, Seattle, WA) was used to analyze nCounter data. Data were normalized to the geometric mean of the reference genes: actin-beta, cyclophilin A, and hypoxanthine phosphoribosyltransferase 1 (Table 2), and results are shown in Figs. 4-42. A relative expression of 20 was the limit of detection. 
Table 2

List of genes analyzed from longissimus muscle tissue of Angus- and Wagyu-sired steers

\begin{tabular}{|c|c|c|}
\hline Gene abbreviation & Gene name & Accession number \\
\hline ACACA & acetyl-CoA carboxylase alpha & NM_174224.2 \\
\hline ACACB & acetyl-CoA carboxylase beta & NM_001205333.1 \\
\hline ACADVL & acyl-CoA dehydrogenase very long chain & NM_174494.2 \\
\hline ACLY & ATP citrate lyase & NM_001037457.1 \\
\hline AGPAT1 & 1-acylglycerol-3-phosphate 0-acyltransferase 1 & NM_177518.1 \\
\hline ANGPT1 & angiopoietin 1 & NM_001076797.1 \\
\hline ANGPT2 & angiopoietin 2 & NM_001098855.1 \\
\hline CD36 & cd36 molecule (thrombospondin receptor) & NM_174010.2 \\
\hline CEBPA & CCAAT enhancer binding protein alpha & NM_176784.2 \\
\hline CEBPB & CCAAT enhancer binding protein beta & NM_176788.1 \\
\hline CPAT1A & carnitine palmitoyltransferase $1 \mathrm{~A}$ & NM_001304989.2 \\
\hline CREB1 & cAMP responsive element binding protein 1 & NM_174285.1 \\
\hline DBI & diazepam binding inhibitor & NM_001113321.1 \\
\hline DGAT1 & diacylglycerol 0-acyltransferase 1 & NM_174693.2 \\
\hline DLK1 & delta like non-canonical notch ligand 1 & NM_174037.2 \\
\hline ELOVL6 & fatty acid elongase 6 & NM_001102155.1 \\
\hline FABP4 & fatty acid binding protein 4 & NM_174314.2 \\
\hline FASN & fatty acid synthase & NM_001012669.1 \\
\hline FBLN1 & fibulin 1 & NM_001098029.1 \\
\hline GPAM & glycerol-3-phosphate acyltransferase & NM_001012282.1 \\
\hline GPD1 & glycerol-3-phosphate dehydrogenase 1 & NM_001035354.1 \\
\hline LDLR & low density lipoprotein receptor & NM_001166530.1 \\
\hline LIPE & lipase E, hormone sensitive type & NM_001080220.1 \\
\hline LPIN1 & lipin 1 & NM_001206156.2 \\
\hline LPL & lipoprotein lipase & NM_001075120.1 \\
\hline MGL & monoglyceride lipase & NM_001206681.1 \\
\hline OLR1 & oxidized low density lipoprotein receptor 1 & NM_174132.2 \\
\hline
\end{tabular}




\begin{tabular}{|c|c|c|}
\hline Gene abbreviation & Gene name & Accession number \\
\hline PDGFRA & platelet-derived growth factor receptor alpha & NM_001192345.1 \\
\hline PDGFRB & platelet-derived growth factor receptor beta & NM_001075896.2 \\
\hline PNPLA2 & patatin like phospholipase domain containing 2 & NM_001046005.2 \\
\hline PPARA & peroxisome proliferator activated receptor alpha & NM_001034036.1 \\
\hline PPARD & peroxisome proliferator activated receptor delta & NM_001083636.1 \\
\hline PPARG & peroxisome proliferator activated receptor gamma & NM_181024.2 \\
\hline PPARGC1A & PPARG coactivator 1 alpha & NM_177945.3 \\
\hline PTGIS & prostaglandin I2 synthase & NM_174444.1 \\
\hline PTGS1 & prostaglandin-endoperoxide synthase 1 & NM_001105323.1 \\
\hline PTGS2 & prostaglandin-endoperoxide synthase 2 & NM_174445.2 \\
\hline SCARB1 & scavenger receptor class $\mathrm{B}$ member 1 & NM_174597.2 \\
\hline SCD & stearoyl-CoA desaturase & NM_173959.4 \\
\hline SLC27A1 & solute carrier family 27 member 1 & NM_001033625.2 \\
\hline SREBF1 & sterol regulatory element binding transcription factor 1 & NM_001113302.1 \\
\hline VEGFA & vascular endothelial growth factor $A$ & NM_174216.2 \\
\hline ZFP423 & zinc finger protein 423 & NM_001101893.1 \\
\hline ACTB & actin beta & NM_173979.3 \\
\hline EEF1A2 & eukaryotic translation elongation factor 1 alpha 2 & NM_001037464.1 \\
\hline HPRT1 & hypoxanthine phosphoribosyltransferase 1 & NM_001034035.1 \\
\hline PPIA & peptidylprolyl isomerase $\mathrm{A}$ & NM_178320.2 \\
\hline PPP1CA & protein phosphatase 1 catalytic subunit alpha & NM_001035316.2 \\
\hline SDHA & succinate dehydrogenase complex flavoprotein subunit $A$ & NM_174178.2 \\
\hline
\end{tabular}

\section{Statistical Analysis}

Statistical analyses were performed using PROC MIXED in SAS (SAS Inst. Inc., Cary, NC). The experimental designs of both experiment 1 and 2 were randomized complete block design with animal as the experimental unit. Prior to cellularity analysis, PROC UNIVARIATE was used to determine the proportion of IM adipocytes present within histogram bins at $500 \mu \mathrm{m}^{2}$ intervals. The statistical model used for cellularity and gene expression analyses was: $Y_{i j}=\mu+T_{i}+B_{j}+T B_{i j}+e_{i j}$, where $T_{i}=$ treatment, $B_{j}$ $=$ biopsy collection, $\mathrm{TB}_{\mathrm{ij}}$ is the interaction as a fixed effect, and $\mathrm{e}_{\mathrm{ij}}=$ random error. The REPEATED 
statement was used to determine the effect of time (biopsy collection) on gene expression, and the covariance structure with the lowest BIC was used. The LSMEANS, PDIFF, and SLICE statements were used to record treatment least square mean estimates, standard errors (SEM), and distinguish differences between the treatment levels. A significance of fixed effects was established at $P \leq 0.05$ and tendencies are discussed at $0.05<P \leq 0.15$.

\section{Results And Discussion}

In experiment 1 , 12th rib LM IM fat percentage, determined by ether extraction, tended $(P=0.13)$ to be greater for WA-MD steers compared with AN and WA-GD steers (12.7 vs. 9.7, 8.8 \%, respectively). In experiment 2, 12th rib LM IM fat percentage, was greater $(P \leq 0.01)$ for WA-MB steers compared with AN and WA-GB steers (15.0 vs. 9.7, 8.2 \%, respectively). Overall, steers sired by OW Yasufuku 229Y, the Wagyu bull selected for high marbling genetic potential, had a greater percentage of IM fat at harvest compared with steers sired by GAR Sunrise, the Angus bull selected for high marbling genetic potential, and LMR Fukutsuru 729T, the Wagyu bull selected for high growth genetic potential (Jaborek et al., 2020).

\section{Intramuscular adipocyte cellularity}

The distribution of IM adipocytes, from steers with a similar age and DOF in experiment 1 and similar BW in experiment 2, exhibited a unimodal distribution with a positive skew (Figs. 1A, 1B). In experiment 1, AN steers had a greater $(P<0.04)$ proportion of IM adipocytes with an area between 1,500 to $2,000 \mu \mathrm{m}^{2}$ at 205 DOA compared with WA-MD steers (treatment $\times$ time interaction; Fig. 2A). Additionally, WA-GD and WA-MD steers had a greater $(P<0.02)$ proportion of IM adipocytes with an area between 12,500 to 13,000 $\mu \mathrm{m}^{2}$ at 513 DOA compared with AN steers, with WA-GD tending $(P=0.07)$ to have a greater proportion of IM adipocytes with an area between 11,500 to $12,000 \mu \mathrm{m}^{2}$ at 513 DOA compared with AN steers (Fig. 2D). Between the different treatments, AN steers tended to have a lesser proportion of IM adipocytes with an area between 6,500 to $7,000 \mu \mathrm{m}^{2}(P=0.06), 7,000$ to $7,500 \mu \mathrm{m}^{2}(P=0.14), 8,500$ to $9,000 \mu \mathrm{m}^{2}(P=$ 0.15) compared with WA-MD steers, 9,500 to $10,000 \mu \mathrm{m}^{2}(P=0.15)$ compared with WA-GD and WA-MD steers, and 10,000 to $10,500 \mu \mathrm{m}^{2}(P=0.11), 12,500$ to $13,000 \mu \mathrm{m}^{2}(P=0.07)$, and greater than $13,000 \mu \mathrm{m}^{2}$ $(P=0.11)$ compared with WA-GD steers. Over time, animal age affected $(P<0.04)$ the proportion of IM adipocytes in each $500 \mu \mathrm{m}^{2}$ interval, except between 2000 to $2500 \mu \mathrm{m}^{2}(P=0.33$; Fig. 1A). In general, at 205,268 , and 331 DOA there was a greater proportion of IM adipocytes between 0 to $2,000 \mu \mathrm{m}^{2}$ and at 531 DOA there was a greater proportion of IM adipocytes between 4,500 to greater than $13,000 \mu \mathrm{m}^{2}$.

In experiment 2, WA-GB and WA-MB steers had a greater $(P<0.03)$ proportion of IM adipocytes with an area between 12,500 to $13,000 \mu \mathrm{m}^{2}$ at $613 \mathrm{~kg}$ compared with AN steers, and a tendency for WA-GB steers to have the greatest proportion of IM adipocytes with an area between 5,500 to $6,000 \mu \mathrm{m}^{2}(P=0.12)$ and 9,500 to $10,000 \mu \mathrm{m}^{2}(P=0.12)$ at $613 \mathrm{~kg}$ (treatment $\times$ time interaction; Fig. 3D). Likewise, WA-GB steers tended to have a greater proportion of IM adipocytes with an area between 5,500 to $6,000 \mu \mathrm{m}^{2}(P=0.12)$ at $296 \mathrm{~kg}$ (Fig. 3B). Between the different treatments, AN steers had a lesser or tended to have a lesser 
proportion of IM adipocytes with an area between 4,000 to $4,500 \mu \mathrm{m}^{2}(P=0.11), 4,500$ to $5,000 \mu \mathrm{m}^{2}(P=$ $0.08), 6,500$ to $7,000 \mu \mathrm{m}^{2}(P=0.14), 7,000$ to $7,500 \mu \mathrm{m}^{2}(P=0.10), 8,000$ to $8,500 \mu \mathrm{m}^{2}(P=0.13)$ compared with WA-GB steers and 8,500 to $9,000 \mu \mathrm{m}^{2}(P<0.03), 9,500$ to $10,000 \mu \mathrm{m}^{2}(P<0.01), 10,000$ to $10,500 \mu \mathrm{m}^{2}(P=0.06)$, and greater than $13,000 \mu \mathrm{m}^{2}(P=0.07)$ compared with WA-GB and WA-MB steers. Over time, the proportion of IM adipocytes in each $500 \mu \mathrm{m}^{2}$ interval was different $(P<0.02)$ when steers were compared at a similar BW (Fig. 1B). In general, when steers weighed 241, 296, and $400 \mathrm{~kg}$ there was a greater proportion of IM adipocytes between 0 to 2,000 $\mu \mathrm{m}^{2}$ and when steers weighed $613 \mathrm{~kg}$ there was a greater proportion of IM adipocytes between 4,500 to greater than $13,000 \mu \mathrm{m}^{2}$.

A pitfall in the present study was the lack of experimental units to provide enough power to detect statistical differences for the cellularity results. The SEM for each level within the treatment $\times$ time interaction varied considerably ( 0.04 to 7.99 and 0.03 to 6.89 in experiments 1 and 2, respectively), with it decreasing as the proportion of cells in each $500 \mu^{2}$ interval decreased and cell size increased. It was hypothesized that the treatment $\times$ time interaction for the cellularity distributions would differ more frequently, which would help explain differences in marbling deposition between Angus and Wagyu cattle. Therefore, the distribution of IM adipocyte area will be interpreted further by treatment and biopsy collection. In experiment 1, at 205 DOA (Fig. 2A), WA-MD steers had IM adipocytes that were less mature compared with AN and WA-GD steers, which is illustrated by a greater proportion (+ $8 \%)$ of small IM adipocytes $\left(0\right.$ to $\left.500 \mu \mathrm{m}^{2}\right)$ and a lesser proportion of larger IM adipocytes $\left(1,000\right.$ to $\left.2,000 \mu \mathrm{m}^{2}\right)$. At the second biopsy time point (268 DOA; Fig. 2B), it appears WA-GD steers developed a greater proportion (+ $10 \%$ ) of small IM adipocytes ( 0 to $500 \mu \mathrm{m}^{2}$ ) relative to AN and WA-MD steers. A greater proportion of smaller IM adipocytes for WA-MD and WA-GD steers at 205 and 268 DOA, respectively, may indicate the proliferation of new smaller IM adipocytes. At 331 DOA (Fig. 2C, WA-GD and WA-MD steers shift to a greater proportion of larger IM adipocytes (1,500 to 3,000 $\left.\mu \mathrm{m}^{2}\right)$, but interestingly AN steers appeared to retain a portion of smaller IM adipocytes $\left(1,000\right.$ to $\left.1,500 \mu \mathrm{m}^{2}\right)$ while increasing the proportion of larger IM adipocytes (2,000 to 6,500 $\mu^{2}$ ). At harvest (513 DOA; Fig. 2D), the distribution of IM adipocyte area was very similar between steers in different treatments. Indicating that marbling differences between the treatments at harvest were likely due to treatment differences in IM adipocyte number rather than IM adipocyte size.

In experiment 2, the distribution of IM adipocytes from the first biopsy ( $241 \mathrm{~kg}$; Fig. $3 \mathrm{~A}$ ) is very similar to experiment 1 , as steers were biopsied on the same day to initiate the present study. Unlike in experiment 1, at the second biopsy ( $246 \mathrm{~kg}$; Fig. 3B) in experiment 2, IM adipocytes from WA-GB steers began to shift towards a greater proportion of larger IM adipocytes with an area between 1,000 to 2,000 $\mu \mathrm{m}^{2}$ when compared with AN and WA-MB steers. At $400 \mathrm{~kg}$ (Fig. 3C) WA-GB and WA-MB steers continued to demonstrate a shift towards a greater proportion of larger IM adipocytes $\left(2,500\right.$ to $\left.4,000 \mu \mathrm{m}^{2}\right)$ when compared with AN steers, while AN steers still had a greater proportion of smaller IM adipocytes $(1,000$ to $\left.1,500 \mu \mathrm{m}^{2}\right)$. At harvest (613 kg; Fig. 3D), the distribution of IM adipocyte area would indicate that WA-GB 
steers have a slightly larger IM adipocyte area on average due to the distribution being shifted slightly towards the right.

Two techniques are commonly used to determine adipocyte cellularity: 1) IM adipocytes are measured within the muscle tissue or 2) IM adipocytes are dissected and extracted from the muscle and subsequently measured. Due to the irregular shape of IM adipocytes found within the muscle tissue, IM adipocyte size is reported by adipocyte area in the present study, which makes comparisons to reports of adipocyte diameter somewhat difficult. Regardless, many of the IM adipocyte distributions that are reported (Schiavetta et al., 1990; Albrecht, et al., 2011; Yamada and Nakanishi, 2012) have a greater proportion of medium-sized IM adipocytes with a normal distribution. When IM adipocyte distributions from the present study are presented by diameter rather than area, they also show a greater proportion of medium-sized IM adipocytes with a normal distribution. Albrecht et al. (2011) reported a 10 to $20 \mu \mathrm{m}$ smaller size difference in IM adipocyte diameter between intact and dissected IM adipocyte measurements, which may explain the smaller IM adipocyte size observed in the present study. Interestingly, others (Hood and Allen, 1973; Schoonmaker et al., 2004, Gorocica-Buenfil et al., 2007; Pickworth et al., 2011; Pickworth et al., 2012) reported bimodal IM adipocyte distributions, in addition to the larger proportion of medium-sized adipocytes, a new cluster of small adipocytes were recruited from preadipocytes. Robelin (1981) and Schoonmaker et al. (2004) proposed that a new wave of preadipocytes are recruited to form a new cluster of small-sized adipocytes once the average adipocyte diameter reaches approximately $90 \mu \mathrm{m}$. However, when the mean IM adipocyte diameter is compared between the study reported by Schoonmaker et al. (2004) and others (Gorocica-Buenfil et al., 2007; Pickworth et al., 2011; Pickworth et al., 2012), mean IM adipocyte diameter was smaller than $90 \mu \mathrm{m}$, but IM adipocytes already exhibited a bimodal distribution. This may be due to the breed of cattle used in the study, as Schoonmaker et al. (2004) used Holsteins and the other studies (Gorocica-Buenfil et al., 2007; Pickworth et al., 2011; Pickworth et al., 2012) used SimAngus crossbred cattle. Additionally, the LM lipid content was lesser for Holstein steers reported by Schoonmaker et al. (2004) when compared with other studies (Gorocica-Buenfil et al., 2007; Pickworth et al., 2011; Pickworth et al., 2012), indicating major differences in IM adipocyte number contributing to the differences in lipid content deposited in the LM. When comparing IM adipocyte size between Wagyu and Holstein steers, both breeds demonstrate hypertrophy with increasing IM adipocyte size from 10 to 18 months of age, but IM adipocytes remained at a similar size from 18 to 26 months of age (Albrecht et al., 2011). Results from the present study are in agreement, as IM adipocyte size continued to increase from 205 DOA until harvest $(513,536$, and 591 DOA for AN steers, WA-GB steers, and WA-MB steers, respectively). Unique IM adipocyte distributions have been reported in older cattle by May et al. (1994; 34 and 26 months of age) and Kruk et al. (2018; 22 months of age), where a very large proportion of new small IM adipocytes were recruited to produce a IM adipocyte distribution with a very strong positive skew. May et al. (1994) reported a greater proportion of smaller IM adipocytes (from 20 to $40 \mu \mathrm{m}$ ) for crossbred Wagyu steers, which had a greater marbling score at harvest, compared with Angus steers (53 vs. $47 \%$, respectively). Kruk reported $46 \%$ of IM adipocytes with a diameter ranging from 10 to $30 \mu \mathrm{m}$. Based on the lack of a bimodal distribution indicating preadipocyte recruitment for the formation of a cluster of small-sized IM adipocytes in the 
present study, we believe that an influx of small-sized IM adipocytes was forthcoming if steers had remained on feed for another month or two. The gene expression results provide further support to this hypothesis and will be discussed later.

\section{Adipogenic gene expression}

Pre-adipocyte determination, proliferation, and the differentiation of pre-adipocytes into mature adipocytes is recognized as the process of adipogenesis, which is regulated by a complex signaling cascade. The expression of zinc finger protein 423 (ZFP423) in mesenchymal progenitor cells represents determination or commitment to the adipose lineage and presence of preadipocytes (Gupta et al., 2010; Haung et al., 2012). However, ZFP423 expression will continue to increase after adipocyte differentiation occurs and ZFP423 will no longer act as a good marker to identify preadipocytes in mature adipose tissue. The expression of delta like non-canonical notch ligand 1 (DLK1), also called preadipocyte factor 1 (Pref-1), inhibits CCAAT enhancer binding protein beta (CEBPB) and delta (CEBPD) expression needed for pre-adipocyte differentiation and has been recognized as a pre-adipocyte marker (Hudak and Sul, 2013). Fibulin 1 (FBLN1) is a glycoprotein associated with the extracellular matrix and has recently been identified as an IM pre-adipocyte marker in muscle, as well as prostacyclin synthase (PTGIS; Kichoon Lee, personal communication). Prostaglandin $\mathrm{I}_{2}$ (prostacyclin) has been reported to initiate differentiation in pre-adipocytes by triggering CAMP release, leading to subsequent CAMP responsive element binding protein 1 (CREB1) activation, and leading to the eventual upregulation of early differentiation transcription factors, such as CEBPB and CEBPD (Aubert et al., 2000; Belmonte et al., 2001). Activation of CREB1 by both protein kinase $A$ (PKA) and extracellular signal-regulated kinase (ERK) are needed for CREB1 to promote CEBPB and CEBPD expression (Belmonte et al., 2001). Additionally, peroxisome proliferator activated receptor delta (PPARD) has been reported to be upregulated by CAMP signaling, which leads to mitotic expansion of pre-adipocytes and assists with the subsequent upregulation of peroxisome proliferator activated receptor gamma (PPARG; Bastie et al., 1999; Hansen et al., 2000). The expression of PPARD can also upregulate genes associated with fatty acid transport and uptake for betaoxidation in the LM (Bastie et al., 1999; Holst et al., 2003). After mitotic expansion occurs, CEBPB works to upregulate late differentiation transcription factors such as PPARG by binding to promoters on the DNA (Wu, et al., 1996; Tang et al., 2003). Upregulation of PPARG can upregulate CCAAT enhancer binding protein alpha (CEBPA) to activate insulin-dependent signaling, where the two transcription factors work synergistically to advance terminal differentiation and the upregulation of other genes associated with lipogenesis (Wu et al., 1999; Tang et al., 2003).

In experiment 1, ZFP423 expression increased over time $(P \leq 0.01)$, with an upregulation at 331 and 442 DOA, with no differences ( $P=0.73$ ) observed between steers in different treatments (Fig. 4A). Expression of DLK1 was not different between treatments $(P=0.99)$ and only tended $(P=0.14)$ to be greater at the fifth biopsy collection. Similarly, FBLN1 expression was greater $(P \leq 0.01)$ at 513 DOA for steers in the present study (Fig. 5A). The expression of PTGIS was greater $(P \leq 0.01)$ at 205 and 513 DOA than at 331 and 422 DOA, with no differences ( $P=0.37$ ) observed between steers in different treatments (Fig. 6A). There was a greater $(P \leq 0.02)$ CREB1 expression at $422 \mathrm{DOA}$ and up to $513 \mathrm{DOA}$, but there also tended to 
be a treatment $\times$ biopsy interaction $(P=0.10)$ where AN steers tended to have a greater CREB1 expression at 422 DOA compared with WA-MD steers (Fig. 7A). The expression of PPARD (Fig. 8A) and CEBPB (Fig. 9A) had significant treatment $\times$ biopsy interactions. Instead of being down regulated at 268 DOA, PPARD expression was sustained at a greater (treatment $\times$ biopsy interaction, $P \leq 0.01$ ) level by WA-MD steers compared with AN and WA-GD steers. Additionally, AN steers had a greater (treatment $\times$ biopsy interaction, $P \leq 0.01)$ PPARD expression compared with WA-GD and WA-MD steers at 422 DOA and WAGD steers at 513 DOA. In experiment 1, AN steers exhibited a greater (treatment $\times$ biopsy interaction, $P \leq$ $0.01)$ CEBPB expression at 422 DOA compared with WA-GD and WA-MD steers. The expression of CEBPA was near the limit of detection in the present study, but demonstrated a slight upregulation $(P \leq 0.01)$ at 422 and $513 \mathrm{DOA}$, and tended $(P=0.15)$ to be greater for WA-MD steers compared with WA-GD steers (Fig. 10A). Expression of PPARG was greater $(P \leq 0.01)$ at 422 and 513 DOA; while WA-MD steers had a greater $(P \leq 0.04)$ PPARG expression, specifically at 331, 422, and 513 DOA, when compared with AN and WA-GD steers (Fig. 11A)

In experiment 2, ZFP423 expression demonstrated a similar pattern as in experiment 1 , with a greater ( $P$ $\leq 0.01)$ ZFP423 expression when steer body weight was 276 and $531 \mathrm{~kg}$ (Fig. 4B). Expression of DLK1 was not different between treatments $(P=0.29)$ or biopsy collections $(P=0.26)$. Similar to experiment 1 , FBLN1 expression was greatest $(P \leq 0.01)$ at harvest $(613 \mathrm{~kg})$, intermediate when steers weighed $400 \mathrm{~kg}$ and $531 \mathrm{~kg}$, and least at 296 and $241 \mathrm{~kg}$, with WA-MB steers tending $(P=0.07)$ to have a greater FBLN1 expression compared with AN steers (Fig. 5B). The expression of PTGIS $(P \leq 0.01)$ was down regulated at $276 \mathrm{~kg}$, but was upregulated again at $613 \mathrm{~kg}$, which was similar to experiment 1 (Fig. 6B). Expression of CREB1 exhibited a treatment $\times$ biopsy interaction $(P \leq 0.02)$, where AN steers had a greater expression at $531 \mathrm{~kg}$ compared with WA-GB and WA-MB steers, and AN steers also tended to have a greater CREB1 expression at $400 \mathrm{~kg}$ compared with WA-MB steers (Fig. 7B). There was a treatment $\times$ biopsy interaction for PPARD expression $(P \leq 0.01)$, where WA-MB steers exhibited approximately 2 times greater expression of PPARD at $400 \mathrm{~kg}$ compared with AN and WA-GB steers, while AN steers had a slightly greater PPARD expression than WA-GB and WA-MB steers at $531 \mathrm{~kg}$ (Fig. 8B). The expression of CEBPB was upregulated $(P \leq 0.01)$ at $613 \mathrm{~kg}$, but tended to exhibit a treatment $\times$ biopsy interaction $(P=0.15)$, where AN steers tended to have a greater expression at $531 \mathrm{~kg}$ compared with WA-MB steers (Fig. 9B). Additionally, WAGD and WA-MD steers tended to have a greater CEBPB expression at $296 \mathrm{~kg}$ compared with AN steers, and WA-MD steers tended to have a greater expression at $400 \mathrm{~kg}$ compared with AN steers. The expression of CEBPA was not different across treatment or time, likely due to the low level of detection for this gene. The expression of PPARG tended to display a treatment $\times$ biopsy interaction $(P=0.09)$, where WA-MB steers had a greater PPARG expression at $400 \mathrm{~kg}$ compared with AN and WA-GB steers (Fig. 11B). Additionally, PPARG expression was greater $(P \leq 0.01)$ at 531 and $613 \mathrm{~kg}$, and tended $(P=0.09)$ to be greater on average for WA-MB steers compared with AN and WA-GB steers.

Overall, ZFP423 expression demonstrated an increased commitment of mesenchymal progenitor cells to the adipogenic lineage or adipogenic ability within the LM tissue of steers of the present study. Along with a greater IM fat percentage, a greater expression of ZFP423 was reported in the sternomandibularis muscle by Duarte et al. (2013) for Wagyu steers compared with Angus steers at 24 months of age. In 
younger (12 mo.) heifers, Fu et al. (2017) reported Wagyu heifers tended to have a greater ZFP423 expression compared with Angus heifers in the biceps femoris muscle. However, the results from the present study do not support this theory as no breed differences were observed between Angus and Wagyu steers.

Expression of DLK1 was relatively constant across the five biopsy collections, with no treatment differences observed. In agreement, Pickworth et al. (2011) reported no difference in DLK1 expression at harvest between high and low marbling steers. In disagreement, Albrecht et al. (2015) believe they were able to properly identify pre-adipocytes by using DLK1 as a marker in the LM of a 6 month old bovine fetus and LM tissue from 26 month old Holstein and Wagyu steers. A greater number of DLK1 positive cells were identified in Holstein steers compared with Wagyu steers at 26 months of age, and a greater overall mean DLK1 expression in Holstein steers compared with Wagyu steers across all biopsy collections (10, 14, 18, 22, and 26 months of age; Albrecht et al., 2015). Expression of DLK1 has been reported in skeletal muscle, particularly satellite cells, and promotes myogenic differentiation for muscle regeneration and hypertrophy (Waddell et al., 2010). Therefore, it is impossible to determine the contribution of DLK1 as a pre-adipocyte marker due to the combined presence of pre-adipocytes and satellite cells within the muscle samples collected in the present study.

As a potential pre-adipocyte marker, a greater FBLN1 expression at harvest could indicate a greater number of pre-adipocytes present in the LM of steers from the present study. The expression of FBLN1 is further supported by PTGIS expression. The expression of PTGIS exhibited two peaks, at the time of weaning and harvest, approximately 6.7 months of age and 16.8 to 19.4 months of age, respectively. The creation of prostacyclin from arachidonic acid requires cyclooxygenase 1/2 (COX1/2), also called prostaglandin-endoperoxide synthase 1/2 (PTGS1/2), both COX1 and 2 were near or below the level of detection in the present study. Rahman et al. (2014) demonstrated that PTGIS and IP receptor expression closely mirrored the 6-keto-PGF $1 \mathrm{a}$ (a stable product of prostacyclin) concentration in 3WA-MD-L1 cells, where expression was greatest in pre-adipocytes growing to confluence and in maturing adipocytes, but not differentiating pre-adipocytes. Therefore, prostacyclin from pre-adipocytes or mature adipocytes can impose an autocrine or paracrine effect to initiate differentiation (Darimont et al., 1994; Rahman, 2019). It may be that a cluster of pre-adipocytes were beginning to initiate differentiation at the time of the first biopsy through an autocrine signal and at the fifth biopsy mature adipocytes were sending a paracrine signal to pre-adipocytes to begin differentiation again to form a new cluster of small adipocytes. The hypothesis of a new cluster of small adipocytes forming shortly after the time at which cattle were harvested in the present study could help explain the lack of a bimodal distribution observed with the IM adipocyte cellularity results.

Expression of CREB1 was greater for Angus-sired steers at the third and fourth biopsies, approximately 12.1 and 16.8 months of age, compared with Wagyu-sired steers. It appears as if a greater CREB1 expression by Angus-sired steers could be upregulating CEBPB at the fourth biopsy collection compared with Wagyu-sired steers. Noticeably, Wagyu-sired steers did not demonstrate any peaks in CREB1 
expression, which may indicate the transient nature of CREB1 activation for CEBP upregulation (Reusch et al., 2000).

A greater expression of CEBPB was observed at the fifth biopsy for all steers. At $296 \mathrm{~kg}$ and $400 \mathrm{~kg}$, WAMB steers tended to have a greater CEBPB expression compared with Angus-sired steers, and Angus-sired steers had a greater CEBPB expression at the fourth biopsy collection (approximately 13.8 months of age) compared with Wagyu-sired steers. This CEBPB expression may indicate that IM adipocytes from WA-MB steers had initiated differentiation at a younger age and lesser BW compared with other steers in the present study. In a study conducted by Wang et al. (2009), Wagyu- and Piedmontese-sired heifers were raised on pasture until entering the feedlot around 26 months of age at approximately $450 \mathrm{~kg}$. The CEBPB expression of Wagyu- and Piedmontese-sired heifers was greatest at 12 months of age before preceding the upregulation of PPARG at 25 months of age with sustained expression until harvest at 30 months of age. Moisa et al. (2014) did not observe a peak for CEBPB expression in steers early weaned at 4.6 months of age, but reported peak CEBPB expression at 10 months of age in steers that were creep-fed and weaned at 7 months of age. The major difference between the studies of Wang et al. (2009), Moisa et al. (2014), and the present study, would be nutritional management resulting in different maturity rates for the cattle in those studies. Moisa et al. (2014) used the most intensive management system with early weaning and creep feeding, with both systems exhibiting very different adipogenic patterns, followed by the present study, and lastly, the extensive management system used by Wang et al. (2009). The age at which cattle begin to consume a high-energy diet will influence the timing and duration of the expression of adipogenic genes in the development of marbling.

Steers sired by the Wagyu sire selected for genetic marbling potential (WA-MD and WA-MB) demonstrated unique peaks of PPARD expression at the second and third biopsy in experiment 1 and 2 , respectively. Based on the lack of a bimodal distribution for IM adipocyte area to indicate recent pre-adipocyte proliferation, the unique PPARD peaks may indicate a greater transportation and uptake of fatty acids used for triglyceride formation and PPARG upregulation in the LM of WA-MD and WA-MB steers.

As a possible result of PPARD upregulation in the LM, PPARG expression was greater for WA-MD and WAMB steers beginning at the third biopsy, at approximately 12.1 months of age, compared with steers from other treatments. The expression of PPARG was greater for all steers at the fourth and fifth biopsies when steers were 13.8 months of age or greater. A greater PPARG expression at this time likely upregulated other genes associated with triglyceride synthesis and lipogenesis to enhance marbling deposition. In support, Duarte et al. (2013) and Fu et al. (2017) reported a greater PPARG expression in the muscle of Wagyu cattle compared with Angus cattle. Additionally, Wang et al. (2009) reported a much greater PPARG expression for Wagyu-sired heifers compared with Piedmontese-sired heifers arising just prior to the finishing phase at 25 months of age. It could appear that the consumption of a high-energy diet stimulated PPARG expression when steers were transitioned to the finishing diet at 377 DOA (12.4 months of age) in the present study, and for early-weaned steers in the Moisa et al. (2014) study. However, Wang et al. (2009) reported an upregulation of PPARG in heifers 1 month prior to feedlot entry and Moisa et al. (2014) reported no obvious upregulation of PPARG in normal-weaned, creep-fed steers. 
Therefore, instead of being regulated by the onset of consuming of a high-energy diet or chronological age, the timing of PPARG expression may be related to growth rate and physiological maturity. In agreement with this notion, Graham et al. (2009) and McKiernan et al. (2009) reported a greater percentage of IM fat (3.05 vs. $2.64 \% ; 4.15$ vs. $3.61 \%$, respectively) in the LM and a younger age at harvest (677 vs. 848 DOA; 595 vs 753 DOA, respectively) from steers with a fast post-weaning gain compared with a slow post-weaning gain ( $0.7 \mathrm{vs} .0 .5 \mathrm{~kg} / \mathrm{d})$.

Unfortunately, CEBPA expression was near the limit of detection in the present study. However, the data did exhibit a greater CEBPA expression at the fourth and fifth biopsies in experiment 1 and a tendency for a greater CEBPA expression from WA-MD steers compared with WA-GD steers, similar to PPARG expression. Additionally, Duarte et al. (2013) reported a greater CEBPA expression for Wagyu steers compared with Angus steers at 24 months of age; although, steers were at a much greater age when harvested compared with the present study. As in the present study, Moisa et al. (2014) reported coinciding peaks of CEBPA and CEBPB expression from early weaned steers that appear to slightly follow PPARG expression.

\section{Lipogenesis}

Lipogenesis is the process where energy, in the form of acetyl-CoA, is used to create fatty acids. In cattle, acetate is produced by ruminal fermentation of dietary feedstuffs and can be absorbed into the blood stream from the rumen and transported to peripheral tissues for the production of acetyl-CoA. Additionally, glucose absorbed by adipocytes can produce acetyl-CoA from citrate via ATP citrate lyase (ACLY) in the cytosol. For fatty acid synthesis to occur, acetyl-CoA must be converted to malonyl-CoA by acetyl-CoA carboxylase alpha/beta (ACACA/B) to initiate synthesis of the fatty acid. Fatty acid synthase (FASN) proceeds to add acetyl groups for the formation of 16 and 18 carbon long-chain fatty acids. Fatty acids can be elongated by the addition of two carbons, for example by fatty acid elongase 6 (ELOVL6) or desaturated to include a double bond in the fatty acid by stearoyl-CoA desaturase 1 (SCD1). Sterol regulatory element binding transcription factor 1 (SREBF1) regulates fatty acid synthesis by transcribing ACLY, ACAC, and FASN as well as others (Shimano, 2001). The transcription of SREBF1 is regulated initially by CEBPB and CEBPD, and eventually CEBPA (Payne et al., 2010). Finally, PPARG assists SREBF1 with the regulation of genes, such as GPAM, involved in triglyceride synthesis (Coleman and Lee, 2014).

In experiment 1, SREBF1 expression ( $P \leq 0.01)$ was greater at the first, fourth, and fifth biopsies, at 205, 422 and 513 DOA for steers, respectively (Fig. 12A). Sterol regulatory element binding transcription factor 1 tended to exhibit a treatment $\times$ biopsy interaction $(P=0.051)$, where AN steers had a numerically greater SREBF1 expression at 422 DOA compared with WA-GD and WA-MD steers. The expression of ACLY ( $P \leq$ 0.01; (Fig. 13A), ACACA ( $P \leq 0.01$; (Fig. 14A), and FASN ( $P \leq 0.01$; (Fig. 16A) similarly mirrored SREBF1 expression and peaked at 422 DOA or the fourth biopsy for steers in experiment 1 . The expression of ACACA tended $(P=0.09)$ to be greater for WA-MD steers compared with AN and WA-GD steers, which was driven by its numerically greater expression at 422 DOA. Expression of ACACB was greatest $(P \leq 0.01)$ at 513 DOA and exhibited a treatment $\times$ biopsy interaction $(P \leq 0.04)$, where AN steers had the greatest expression at 422 DOA, WA-GD steers had intermediate expression, and WA-MD steers had the lowest 
expression of ACACB (Fig. 15A). The expression of ELOVL6 ( $P \leq 0.01$; Fig. 17A) and SCD $(P \leq 0.01$; Fig. 18A) were upregulated at 422 DOA and ELOVL6 $(P=0.06)$ and $\operatorname{SCD}(P=0.08)$ tended to be greater for WA-MD steers compared with AN and WA-GD steers. The expression of glycerol-3-phosphate dehydrogenase 1 (GPD1) was greater $(P \leq 0.01)$ at 268, 331, and 422 DOA in steers, while GPD1 became downregulated at 513 DOA (Fig. 19A). The expression of glycerol-3-phosphate acyltransferase (GPAM) exhibited a treatment $\times$ biopsy interaction $(P \leq 0.04)$, where expression at 331 DOA was greater for WAGD and tended to be greater for WA-MD steers compared with AN steers, and WA-MD steers tended to have a greater GPAM expression compared with AN and WA-GD steers at 422 DOA (Fig. 20A). The expression of 1-acylglycerol-3-phosphate 0-acyltransferase 1 (AGPAT1) was slightly greater $(P \leq 0.02)$ at 205 and 513 DOA, but there were no treatment differences (Fig. 21A). A treatment $\times$ biopsy interaction ( $P$ $\leq 0.02$ ) for phosphatidic acid phosphatase or lipin 1 (LPIN1) resulted from WA-MD steers having a numerically greater expression at 268 DOA compared with AN steers, while AN steers had a greater expression at 422 DOA compared with WA-GD and WA-MD steers (Fig. 22A). Expression of LPIN1 closely mirrored the expression of PPARD. Diacylglycerol 0-acyltransferase 1 (DGAT1) expression was relatively constant with the greatest expression tending $(P=0.07)$ to be at 513 DOA (Fig. 23A).

In experiment 2, SREBF1 expression exhibited a treatment $\times$ biopsy interaction $(P \leq 0.01)$, with WA-GB and WA-MB steers having a greater expression at the third biopsy, at $400 \mathrm{~kg}$, but AN steers had a greater expression than WA-GB and WA-MB steers at the fourth biopsy when steers weighed $531 \mathrm{~kg}$ (Fig. 12B). Similar to experiment 1 , steers in experiment 2 had a greater expression of ACLY ( $P \leq 0.01$; Fig. 13B), ACACA ( $P \leq 0.01$; Fig. 14B), FASN ( $P \leq 0.01$; Fig. 16B), ELOVL6 ( $P \leq 0.01$; Fig. 17B), and SCD $(P \leq 0.01$; Fig. 18B) at the fourth biopsy collection or $531 \mathrm{~kg}$. Expression of ACACB was greatest $(P \leq 0.01)$ at 513 DOA and exhibited a treatment $\times$ biopsy interaction $(P \leq 0.01)$, where WA-MB steers had a greater expression at $400 \mathrm{~kg}$ compared with AN and WA-GB steers, and AN steers had a greater expression at 531 $\mathrm{kg}$ compared with WA-GB and WA-MB steers (Fig. 15B). The expression pattern of GPD1 was similar to experiment 1, where GPD1 expression was greater $(P \leq 0.01)$ at the second, third, and fourth biopsies (Fig. 19B). Expression of GPAM exhibited a treatment $\times$ biopsy interaction $(P \leq 0.02)$, with a greater expression for WA-MB steers at 400 and $613 \mathrm{~kg}$ compared with AN and WA-GB steers (Fig. 20B). The expression pattern of AGPAT1 was also similar to experiment 1, where AGPAT1 expression was greater ( $P$ $\leq 0.01$ ) at the first and fifth biopsies, or 241 and $613 \mathrm{~kg}$ (Fig. 21B). Lipin 1 exhibited a similar pattern of expression as PPARD, with a greater expression for WA-MB steers at $400 \mathrm{~kg}$ compared with AN and WAGD steers, and AN steers having a greater expression at $531 \mathrm{~kg}$ compared with WA-GB and WA-MB steers (treatment $\times$ biopsy interaction, $P \leq 0.01$; Fig. 22B). The expression of DGAT1 tended $(P=0.07)$ to be greater for WA-GB and WA-MB steers compared with AN steers.

Overall, SREBF1 expression was upregulated at the fourth and fifth biopsy collections when steers were approximately 13.8 to 19.4 months of age. Angus-sired steers had a greater SREBF1 expression compared with Wagyu-sired steers at 13.8 months of age; however, this did not result in a greater expression of ACLY, ACACA, FASN, ELOVL6, or SCD for Angus-sired steers. Interestingly, in experiment 2, Wagyu-sired steers demonstrated a relatively constant expression across the 5 biopsy time points and still managed to produce a similar expression level for ACLY, ACACA, FASN, ELOVL6, and SCD. At times, 
WA-MD steers tended to have a greater expression of ACACA, ELOVL6, and SCD when compared with Angus-sired steers. Potentially indicating a gene other than SREBF1 may be influencing the expression of ACACA, ELOVL6, and SCD in cattle with a greater marbling potential.

Expression of ACACB was relatively low until harvest and was typically greater for Angus-sired steers at the fourth biopsy collection, with WA-MB steers exhibiting a greater expression at $400 \mathrm{~kg}$ compared with other steers. Unlike the role of ACACA in adipose lipogenesis, ACACB is thought to regulate fatty acid oxidation in the skeletal muscle by the inhibitory effect of malonyl-CoA on CPT1 (McGarry and Brown, 1997). Therefore, the expression pattern of ACACB is more similar to that of the lipolytic genes discussed later in this manuscript. Research is limited on the role of ACACB in marbling deposition of cattle. A tendency for a greater expression of ELOVL6 and SCD by WA-MD and WA-MB steers is in agreement with the fatty acid composition results of cattle used in the present study (Jaborek et al., 2021). In partial agreement, Wang et al. (2009) reported a greater expression of FASN and SCD in Wagyu-sired heifers when compared with the Piedmontese-sired heifers at 25 and 30 months of age. Moisa et al. (2014) reported a greater SREBF1 expression at the final two biopsy collections, when steers were 10.1 and 11.9 months of age, with early-weaned steers having a greater SREBF1 expression compared with normalweaned steers that were creep-fed. Additionally, Moisa et al. (2014) reported a similar expression pattern between ACLY, FASN, ELOVL6, SCD, lipoprotein lipase (LPL), fatty acid binding protein 4 (FABP4), and DGAT2, with early-weaned steers demonstrating a much greater lipogenic gene expression in the LM compared with normal-weaned creep-fed steers; however, their expression appeared to precede the large increase in SREBF1 expression and more closely resembled PPARG expression. It is interesting that normal-weaned steers from the Moisa et al. (2014) study never exhibited an upregulation of lipogenic gene expression even when consuming a high-energy finishing diet and tended to have similar marbling scores at harvest compared with early weaned steers.

The expression pattern of GPAM is similar to PPARG, where WA-MD and WA-MB steers had a greater GPAM expression compared with other steers from 10.9 months of age until harvest (16.3 to 19.4 months of age). In support, De Jager et al. (2013) reported a greater GPAM expression by Wagyu-sired heifers compared with Piedmontese-sired heifers at 25 months of age. Jeong et al. (2012) reported that GPAM was positively and highly correlated $(r=0.74)$ with IM fat percentage in the LM of Korean steers. The first committed step of triglyceride synthesis is regulated by GPAM by creating 1-acylglycerol-3-phosphate, also called lysophosphatidic acid. Next, AGPAT1 adds the second fatty acyl chain to produce phosphatidic acid. Expression of AGPAT1 was relatively constant, with a slightly greater expression at 6.7 and 16.3 to 19.4 months of age. The expression of LPIN1 closely resembled the expression pattern of PPARD or CEBPB, where WA-MD and WA-MB steers had a greater expression at the second and third biopsy collection in experiment 1 and 2, respectively, and Angus-sired steers had a greater expression at the fourth biopsy collection. Therefore, it is possible WA-MD and WA-MB steers could have had a greater concentration of diacylglycerol compared with other steers in the study. Expression of DGAT1 demonstrated very few differences during the present study. Although, Jeong et al. (2012) reported AGPAT1, DGAT1, and DGAT2 were also positively correlated with IM fat percentage in the LM of Korean steers. Unfortunately, DGAT2 was not measured in the present study to determine its effect on triglyceride 
synthesis. Steers (WA-MD and WA-MB) with the greatest percentage of IM fat in the LM, also had either the greatest overall expression of GPAM or a greater expression of LIPN1 early in the feeding period. This correlation between GPAM, LIPN1 and IM fat percentage highlights the importance of these genes and their products, lysophosphatidic acid and diacylglycerol, respectively, in the development and accumulation of marbling in cattle. Lysophosphatidic acid produced by the enzymatic activity of GPAM, can directly activate PPARG (Mclntyre et al., 2003). Meanwhile, phosphatidic acid can inhibit PPARG activation, but LPIN1 is able to restore PPARG activation and its downstream genes by converting phosphatidic acid into diacylglycerol (Zhang et al., 2012). Future research is needed to quantify these intermediate products of triglyceride synthesis in cattle to further assess their ability as potential indicators of marbling potential.

\section{Angiogenesis}

Adipose tissue development, particularly IM fat development, appears to be spatially related to capillary proximity, which was observed during IM adipocyte cellularity analysis. Vascular endothelial growth factor alpha (VEGFA) is necessary for the formation of immature blood vessels in angiogenesis and vasculogenesis (Hausman and Richardson, 2004). Angiopoietin 1 (ANGPT1) is responsible for vascular remodeling and capillary growth and branching, while ANGPT2 is responsible for destabilizing the structure of blood vessels and regulating ANGPT1 (Hausman and Richardson, 2004). Platelet derived growth factors (PDGF), particularly PDGF beta, are responsible for helping to maintain capillary wall stability by the recruitment and proliferation of pericytes (Hausman and Richardson, 2004). Additionally, PDGF helps regulate the proliferation and mitogenic expansion of mesenchymal progenitor cells, and therefore, can inhibit terminal differentiation of adipocytes from occurring (Kim et al., 2015). Uezumi et al. (2010) reported that PDGF receptor alpha (PDGFRA) was a cell surface marker that could be used for identifying mesenchymal progenitor cells determined to the adipogenic lineage.

In experiment 1, VEGFA expression was greater $(P \leq 0.01)$ at 205 and 268 DOA for steers (Fig. 24A). The expression of ANGPT1 was relatively constant from 205 DOA to 422 DOA, but decreased $(P \leq 0.04)$ afterwards (Fig. 25A). The opposite expression pattern was exhibited for ANGPT2, where expression increased $(P \leq 0.01)$ from 205 DOA until 331 DOA and remained relatively constant thereafter (Fig. 26A). The expression of PDGFRA was greater $(P \leq 0.01)$ at the third and fifth biopsy, 331 and 513 DOA, respectively (Fig. 27A). For PDGF receptor beta (PDGFRB) expression, it was greatest $(P \leq 0.01)$ at harvest or 513 DOA (Fig. 28A).

Experiment 2 demonstrated many of the same expression patterns as experiment 1 for the angiogenic genes in the present study. There was a tendency $(P=0.07)$ for a greater VEGFA expression at weaning age (205 DOA), but VEGFA expression remained constant at the subsequent biopsy timepoints (Fig. 24B). Expression of ANGPT1 exhibited a treatment $\times$ biopsy interaction $(P \leq 0.02)$, where ANGPT1 expression was nearly two times greater for AN and WA-GB steers compared with WA-MB steers at $400 \mathrm{~kg}$ (Fig. 25B). Similar to experiment 1 , steers in experiment 2 had a lesser $(P \leq 0.01)$ expression of ANGPT2 at weaning relative to other biopsy collections (Fig. 26B). In addition to the greater $(P \leq 0.02)$ expression of PDGFRA 
at $613 \mathrm{~kg}$, WA-GB steers had a greater $(P \leq 0.01)$ overall mean expression compared with AN and WA-MB steers (Fig. 27B). No differences were detected for PDGFRB expression between steers in experiment 2.

Overall, the expression of VEGFA was greater in the LM at 6.7 to 8.7 months of age, but decreased thereafter. Indicating the development of the vasculature in the LM occurs when cattle are relatively young. The expression of VEGFA tends to be greater in other fat depots, such as the renal, mesenteric, and intermuscular depots, when compared with the IM and subcutaneous fat depots (Yamada et al., 2010). Interestingly, VEGFA expression is greater in the mesenteric and intermuscular fat depots when Wagyu steers consume a high roughage diet rather than a high-concentrate diet, but VEGFA expression was similar in the IM and subcutaneous fat depots across the two diets (Yamada and Nakanishi, 2012). Therefore, it appears VEGFA expression may be upregulated in earlier maturing fat depots such as the renal, mesenteric, and intermuscular depots to accommodate larger adipocytes. It is not known if VEGFA expression would become upregulated in the LM if IM adipocytes reached a greater size than IM adipocytes measured in the present study.

The expression of ANGPT 1 and 2 were relatively constant across the five biopsy collections in the present study, with the lowest ANGPT1 expression at harvest and the lowest ANGPT2 expression at weaning. Previous studies measuring ANGPT expression in the LM of cattle have also reported very few treatment differences (Lancaster et al., 2014; Roberts et al., 2015). It is interesting the slight differences reported in the present study do not support the idea that greater vascularization in the LM would allow for a greater availability of nutrients for growing IM adipocytes. This lack of support could indicate a lack of change in vascularization within the LM at this life stage in cattle. Additional research is needed to clarify the effects of ANGPT on angiogenesis and adipogenesis in cattle. Expression of PDGFR alpha and beta demonstrated a slightly greater upregulation at the time of harvest. The role of PDGFRA being a preadipocyte marker and PDGFRB helping to regulate capillary stability makes interpretation of the results difficult. With the lack of support indicating angiogenic changes in the LM from VEGFA and ANGPT, it is possible an upregulation of PDGFRA expression could support the idea of a forthcoming wave of new pre-adipocytes.

\section{Fatty acid uptake and transport}

Fatty acids derived from dietary feedstuffs or created via de novo fatty acid synthesis in the liver can be transported in the form of triglycerides by chylomicrons and lipoproteins throughout the circulatory system to supply peripheral tissues such as the skeletal muscle and adipose tissue with energy for use and storage. Lipoprotein lipase or other lipoprotein receptors, such as low density lipoprotein receptor (LDLR), hydrolyze triglycerides from these lipoproteins into monoacylglycerol and two fatty acids.

Cholesterol transfer from high density lipoproteins is mediated by scavenger receptor class B member 1 (SCARB1). The cd36 molecule (CD36) and fatty acid transport protein 1 (FATP1), also called solute carrier family 27 member 1 (SLC27A1), transport fatty acids into the cell. Fatty acid transporters such as, FABP4 and acyl-CoA binding protein (ACBP), also called diazepam binding inhibitor (DBI), bind to fatty acids for transport of the fatty acid within the cell. 
In experiment 1, LPL expression was greater $(P \leq 0.01)$ at 205 and 513 DOA for steers (Fig. 29A). The expression of LDLR was greater (treatment $\times$ biopsy interaction, $P \leq 0.01$ ) for WA-MD steers at 422 DOA compared with AN and WA-GD steers (Fig. 30A). Expression of SCARB1 was greater $(P \leq 0.01)$ at 205, 268 , and $331 \mathrm{DOA}$, and decreased afterwards, with AN steers tending $(P=0.07)$ to have a greater SCARB1 expression compared with WA-GD and WA-MD steers (Fig. 31A). The expression of CD36 was greater ( $P$ $\leq 0.01)$ at 513 DOA, while WA-MD steers tended $(P=0.11)$ to have a greater CD36 expression than AN steers (Fig. 32A). The expression of FATP1 was greater (treatment $\times$ biopsy interaction, $P \leq 0.01$ ) for AN steers at 422 DOA compared with WA-GD and WA-MD steers (Fig. 33A). Expression of FABP4 was greater $(P \leq 0.01)$ at 422 and $513 \mathrm{DOA}$, and tended $(P=0.07)$ to be greater for WA-MD steers compared with AN and WA-GD steers (Fig. 34A). The expression of ACBP increased $(P \leq 0.01)$ greatly after 205 DOA, with the greatest expression at 268 and 513 DOA for steers in the present experiment (Fig. 35A).

In experiment 2, LPL expression was greater $(P \leq 0.01)$ at 241 and $613 \mathrm{~kg}$, while AN steers tended $(P=$ 0.13 ) to have a greater mean LPL expression compared with WA-GB steers (Fig. 29B). Low density lipoprotein receptor expression was greater for WA-GB and WA-MB steers at $296 \mathrm{~kg}$ compared with AN steers, and WA-GB steers had a greater expression at $400 \mathrm{~kg}$ compared with AN and WA-MB steers (treatment $\times$ biopsy interaction, $P \leq 0.01$; Fig. 30B). The expression of SCARB1 was greatest $(P \leq 0.01)$ at $296 \mathrm{~kg}$ and decreased thereafter, with AN steers tending $(P=0.06)$ to have a greater expression compared with WA-MB steers (Fig. 31B). Similar to experiment 1, CD36 expression was greatest $(P \leq 0.01)$ at the fifth biopsy collection $(613 \mathrm{~kg})$, and WA-MB steers had a greater $(P \leq 0.03)$ expression of CD36 compared with AN steers (Fig. 32B). Like experiment 1, AN steers had a greater expression of FATP1 at the fourth biopsy collection (531 kg) compared with WA-GB and WA-MB steers (Fig. 33B). Similar to CEBPB expression, WA-GB and WA-MB had a greater expression of FATP1 at the second biopsy collection or 276 $\mathrm{kg}$ compared with AN steers (treatment $\times$ biopsy interaction, $P \leq 0.02$ ). Expression of FABP4 was greatest $(P \leq 0.01)$ at 531 and $613 \mathrm{~kg}$ (Fig. 34B). The expression of ACBP exhibited a treatment $\times$ biopsy interaction $(P \leq 0.05)$, where WA-GB steers had a greater expression at $276 \mathrm{~kg}$ compared with AN and WAMB steers (Fig. 35B).

Overall, LPL expression demonstrated a greater fatty acid uptake within the LM when steers were approximately 6.7 and 16.8 to 19.4 months of age. LPL expression may be a partial indication of a greater fatty acid supply from consuming milk at 6.7 months of age and a greater feed intake of the feedlot finishing ration near the time of harvest. Results of LPL expression from Wang et al. (2009) were very similar to the present study, while Moisa et al. (2014) reported a greater LPL expression in concert with other lipogenic genes for early-weaned steers. Expression of LDLR illustrated breed differences over time for fatty acid uptake; with WA-MD and WA-MB steers typically having a greater LDLR expression from 9.4 to 13.8 months of age compared with Angus-sired steers, and WA-GD and WA-GB steers having a greater LDLR expression relative to Angus-sired steers at 9.2 and 11.6 months of age. The expression of LDLR can be regulated by fatty acids, with saturated fatty acids (SFA) reducing, and polyunsaturated fatty acids (PUFA) increasing LDLR expression, respectively (Woollett et al., 1992). This fact is further supported by a greater PUFA:SFA ratio for WA-MD and WA-MB steers compared with other steers in the present study (Jaborek et al., 2020). A greater expression of LDLR may indicate a greater role of receptor- 
mediated triglyceride hydrolysis for Wagyu-sired steers, while Angus-sired steers tended to have a greater expression of $\mathrm{LPL}$, where triglyceride hydrolysis is not receptor-mediated. The expression of another receptor, oxidized low density lipoprotein receptor 1 (OLR1), was below the limit of detection in the present study, likely an indication of its minor involvement in marbling deposition in the LM.

The expression of SCARB1 was greatest at the second biopsy and decreased thereafter in steers from the present study. Additionally, SCARB1 expression tended to be greater for Angus-sired steers compared with WA-MD and WA-MB steers, which could indicate a greater uptake of cholesterol in the LM. However, previous studies have not reported cholesterol differences due to breed in the dissected LM (Rule et al., 1997; Chung et al., 2006) or intact LM (Wheeler et al., 1987; Brugiapaglia et al., 2014). Rhee et al. (1982) reported that cholesterol content of the LM is not related to marbling score or the percentage of IM fat. However, on a percent lipid basis, LM muscle with lower marbling scores had a greater cholesterol content ( $r=-0.66$; Rhee et al., 1982), indicating that a large proportion of cholesterol in beef LM is present in the structural lipids of the cell membrane.

The upregulation of CD36 did not occur until approximately 16.8 to 19.4 months of age or the time of harvest, which indicates fatty acid uptake within the LM for the steers was likely occurring via another transport protein at an earlier time. Possibly by FATP1, which demonstrated a relatively constant expression for Wagyu-sired steers compared with the low level of expression prior to peak expression at approximately 13.8 months of age for Angus-sired steers. Therefore, Wagyu-sired steers may have a greater fatty acid uptake at a younger age (9.3 months) compared with Angus-sired steers. The expression of CD36 was typically greater for WA-MD and WA-MB steers compared with other steers in the present study. Along with CD36, Jeong et al. (2012) reported that FATP1 was positively correlated with the percentage of IM fat in the LM of Korean steers. These differences for fatty acid transport between Angus- and Wagyu-sired steers may represent differences in energy metabolism within the muscle. Previous research has reported changes in muscle fiber type over time as the animal grows, and as a result, the metabolism of the skeletal muscle is changed in which intramuscular adipocytes reside (Gotoh, 2003). As such, there are reports of intramuscular fat percentage being positively correlated with the percentage of type I muscle fibers (Gotoh, 2003; Hwang et al., 2010; Joo et al., 2017) and negatively correlated with the percentage of type II muscle fibers (Calkins et al., 1981; Ozawa et al., 2000; Gotoh, 2003; Hwang et al., 2010) in the skeletal muscle of cattle (Underwood et al., 2007).

Fatty acid binding protein 4 expression closely resembled PPARG expression, with a large increase in expression after 13.8 months of age and a tendency for a greater FABP4 expression by WA-MD and WAMB steers. In agreement with the present study, Wang et al. (2009) and Moisa et al. (2014) reported an upregulation of FABP4 occurred after peak PPARG expression. Previous reports indicate FABP4 is positively correlated with the percentage of IM fat in the LM (Moore et al., 1991; Jurie et al., 2007). In support of the results in the present study, Duarte et al. (2013) reported a greater number of FABP4 positive cells in the LM from Wagyu steers compared with Angus steers. Additionally, Albrecht et al. (2011) reported that FABP4 expression increased from 10 until 22 months of age, before expression became downregulated at 26 months of age in Japanese Black Wagyu and Holstein steers. The similar 
pattern of expression between PPARG and FABP4 in the present study is likely due to the regulation of FABP4 by PPARG (Tontonoz et al., 1994). Interestingly, greater levels of FABP4 have been reported to trigger proteasomal degradation of PPARG and inhibit adipogenesis, which results in fewer adipocytes and less lipid accumulation by adipocytes (Garin-Shkolnik et al., 2014). The expression of another intracellular fatty acid transporter, ACBP, demonstrated a dramatic increase in expression at the second biopsy collection or after cattle arrived to the feedlot at 8.8 months of age. When steer calves were weaned and switched to a backgrounding diet, ACBP expression became upregulated. The expression of ACBP may be an indication of fatty acid transport in other cells within the LM, such as myocytes, while FABP4 is specific to adipocytes.

\section{Lipolysis}

When energy is needed, fatty acids can be hydrolyzed from triglycerides by adipose triglyceride lipase (ATGL), also called patatin like phospholipase domain containing 2 (PNPLA2), hormone sensitive lipase (HSL), and monoglyceride lipase (MGL). Acyl-CoA bound fatty acids can enter the mitochondria of the cell through carnitine palmitoyltransferases (CPT1/2) to undergo beta-oxidation. Beta-oxidation of fatty acids is initiated by the rate-limiting enzyme, acyl-CoA dehydrogenase (ACAD), for the removal of acetylCoA units for subsequent ATP production. Peroxisome proliferator activated receptor alpha (PPARA) regulates the transcription of genes needed for beta-oxidation.

The expression of PPARA was relatively constant and tended to be greater $(P=0.08)$ at 205 DOA compared with 331 DOA for steers in experiment 1 (Fig. 36A). The expression of PPARG coactivator 1 alpha (PPARGC1A) closely resembled PPARA expression, such that PPARGC1A expression decreased ( $P$ $\leq 0.01$ ) from 205 to 331 DOA (Fig. 37A). Expression of ATGL was upregulated $(P \leq 0.01)$ at 205 and 513 DOA for steers (Fig. 38A). The expression of HSL was greater $(P \leq 0.01)$ at 513 DOA, with WA-MD steers having a greater $(P \leq 0.04)$ overall mean HSL expression compared with AN and WA-GD steers (Fig. 39A). Expression of MGL was greatest $(P \leq 0.01)$ at 513 DOA, and WA-MD steers had a slight tendency (treatment $\times$ biopsy interaction, $P=0.10$; Fig. 40A) to have a greater MGL expression at $422 \mathrm{DOA}$ compared with AN and WA-GD steers. Expression of CPT1 ( $P \leq 0.01$; Fig. 41A) and ACAD very long chain (ACADVL; $P \leq 0.01$; Fig. 42A) were slightly greater at 205 DOA compared with 268, 331, and 442 DOA, with the greatest expression for CPT1 and ACADVL at 513 DOA.

In experiment 2, PPARA expression was greater $(P \leq 0.03)$ for steers at 241,276 , and $613 \mathrm{~kg}$, and tended to be greater $(P=0.10)$ for WA-GB steers compared with AN and WA-MB steers (Fig. 36B). Expression of PPARGC1A was greatest $(P \leq 0.01)$ when steers weighed $241 \mathrm{~kg}$ and decreased thereafter (Fig. 37B). The expression of ATGL $(P \leq 0.01)$ was similar to experiment 1 , with a greater expression at the first and fifth biopsy collections, when steers weighed 241 and $613 \mathrm{~kg}$ (Fig. 38B). The expression of HSL was greater for WA-MB steers at $400 \mathrm{~kg}$ compared with AN and WA-GB steers (treatment $\times$ biopsy interaction, $P \leq$ 0.05 ; Fig. 39B). Expression of MGL $(P \leq 0.01)$ in experiment 2 was greatest at the fifth biopsy when cattle weighed $613 \mathrm{~kg}$ (Fig. 40B). Additionally, WA-MB steers had a greater $(P \leq 0.01)$ mean MGL expression compared with AN and WA-GB steers. Expression of CTP1 was greater $(P \leq 0.01)$ when steers weighed $613 \mathrm{~kg}$, while WA-MB steers tended $(P=0.10)$ to have a greater expression of CPT1 at $400 \mathrm{~kg}$ compared 
with $\mathrm{AN}$ and WA-GB steers (treatment $\times$ biopsy interaction, $P=0.10$; Fig. 41B). The expression of ACADVL was greater for WA-MB steers compared with AN and WA-GB steers at $400 \mathrm{~kg}$, and AN steers had a greater expression at $531 \mathrm{~kg}$ compared with WA-GB steers (treatment $\times$ biopsy interaction, $P=0.07$; Fig. 42B). Additionally, WA-MB steers had a greater $(P \leq 0.03)$ mean ACADVL expression compared with AN and WA-GB steers.

Overall, the regulation of genes responsible for lipolysis by PPARA exhibited a quadratic expression that was greater at the first and fifth biopsy collections, which was 6.7 months of age and 16.8 to 19.4 months of age. Having a similar expression pattern as PPARA, PPARGC1A is likely involved in betaoxidation of fatty acids. A declining expression of lipogenic genes after weaning may be due to dietary transition from a milk-based diet to a total mixed ration of dry ingredients and the site where digestion primarily occurs (small intestine vs. rumen). Additionally, the upregulation of lipolytic genes at the first

and fifth biopsy may have to been partially caused by stress at weaning and fasting prior to harvest. After approximately 14.6 months of age the expression of lipolytic genes (CPT1, ACADVL, ATGL, HSL, and MGL) were upregulated. Jeong et al. (2012) reported negative correlation with the percentage of IM fat in the LM for CPT1, ACADVL, ATGL, and MGL, but a positive correlation with HSL, as did Kazala et al. (2003). Kazala et al. (2003) believe HSL and DGAT enzyme activities within the LM may be related to maturity status of the IM fat depot and used as potential indicators of marbling status, because a greater percentage of IM fat in the muscle is associated with a lesser DGAT and greater HSL activity. Interestingly, this is a similar hypothesis to the one proposed earlier with the regulation of triglyceride synthesis by GPAM and LPIN1 for the production of adipogenic regulatory compounds in lysophosphatidic acid and diacylglycerol. In agreement, the WA-MD and WA-MB steers had a greater percentage of IM fat and overall expression of HSL compared with other steers in the present study. The expression of MGL and CTP1 were greater at times for WA-MD and WA-MB steers compared with other steers; however, expression of MGL and CTP1 were near the limit of detection at the second, third, and fourth biopsy collections. The upregulation of oxidative genes coincided with the upregulation of lipogenic genes as steers matured, which may indicate a change in skeletal muscle metabolism towards becoming more oxidative at the time of harvest.

\section{Conclusions}

Wagyu-sired steers selected for marbling potential had a greater percentage of IM fat in the LM compared with Angus-sired steers selected for marbling and Wagyu-sired steers selected for growth potential. The upregulated expression of PPARD likely led to the upregulation of fatty acid transport proteins and an earlier and overall greater expression of PPARG by Wagyu-sired steers selected for marbling potential. As a result, Wagyu-sired steers selected for marbling potential had a greater expression of GPAM, LPIN1, and HSL, which may assist in upregulating and sustaining adipogenesis by producing adipogenic regulatory compounds in lysophosphatidic acid and diacylglycerol. Furthermore, the combination of IM adipocyte cellularity analysis and gene expression results would indicate that there could be periods of IM adipocyte proliferation prior to the initiation of differentiation occurring around the initiation and termination of the study, at approximately 6.7 months of age and 16.8 to 19.4 months of age of steers in 
the present study. Regardless of genetics, the growth rate of animals, usually influenced by caloric intake, appears to directly influence the timing and accumulation of IM fat in the LM. Feeding strategies may be developed to optimize energy availability during critical times in IM adipocyte development to reduce overall feed costs during the finishing period for cattle feeding operations.

\section{Declarations}

\section{Ethics approval and consent to participate}

Animal procedures and husbandry practices were approved by the Institutional Animal Care and Use Committee (IACUC; protocol number 2015A00000093) of The Ohio State University and followed the guidelines recommended in the Guide for the Care and Use of Agricultural Animals in Agricultural Research and Teaching (FASS, 2010).

\section{Consent for publication}

Not Applicable

\section{Availability of data and material}

The datasets analyzed in the current study are available from the corresponding author on reasonable request.

\section{Competing interests}

The authors declare that they have no competing interests.

\section{Funding}

Salaries and research were supported by state and federal funds appropriated to the Ohio Agricultural Research and Developmental Center (OARDC), The Ohio State University. Samples preparation for cellularity were made at The Comparative Pathology \& Mouse Phenotyping Shared Resource, supported in part by $\mathrm{NCl}$ grant $\mathrm{P} 30 \mathrm{CA} 016058$.

\section{Authors' contributions}

JRJ - contributed to the research design, data collection, data analyses, data interpretation, manuscript construction, and manuscript revision.

FLF - contributed to the research design and manuscript revision.

$\mathrm{KL}$ - contributed to research design.

HNZ - contributed to research design and manuscript revision. 
AER - contributed to research design, data collection, data analyses, data interpretation, and manuscript revision.

\section{Acknowledgements}

The authors would like to acknowledge the farm staff from the Ohio Agricultural Research and Developmental Center (OARDC) feedlot for their help caring for the animals, staff from the Ohio State University (OSU) Meat Science Laboratory for their help with harvesting the cattle, and the Comparative Pathology \& Mouse Phenotyping Shared Resource (CPMPSR) Laboratory for preparing the histology slides for adipocyte cellularity analysis.

\section{References}

1. Albrecht, E., J. Kuzinski, K. Komolka, T. Gotoh, and S. Maak. 2015. Localization and abundance of early markers of fat cell differentiation in the skeletal muscle of cattle during growth - Are DLK1positive cells the origin of marbling flecks? Meat Sci., 100: 237-245.

2. Albrecht, E., T. Gotoh, F. Ebara, J. X. Xu, T. Viergutz, G. Nürnberg, S. Maak, and J. Wegner. 2011. Cellular conditions for intramuscular fat deposition in Japanese Black and Holstein steers. Meat Sci., 891: 13-20.

3. Aubert, J., P. Saint-Marc, N. Belmonte, C. Dani, R. Négrel, and G. Ailhaud. 2000. Prostacyclin IP receptor up-regulates the early expression of $\mathrm{C} / \mathrm{EBP} \beta$ and $\mathrm{C} / \mathrm{EBP} \delta$ in preadipose cells. Mol. Cell. Endocr., 160: 149-156.

4. Bastie, C., D. Holst, D. Gaillard, C. Jehl-Pietri, and P. A. Grimaldi. 1999. Expression of peroxisome proliferator-activated receptor PPARס promotes induction of PPARY and adipocyte differentiation in 3WA-MDC2 fibroblasts. J. Biol. Chem., 274: 21920-21925.

5. Belmonte, N., B. W. Phillips, F. Massiera, P. Villageois, B. Wdziekonski, P. Saint-Marc, J. Nichols, J. Aubert, K. Saeki, A. Yuo, S. Narumiya, G. Ailhaud, and C. Dani. 2001. Activation of extracellular signalregulated kinases and CREB/ATF-1 mediate the expression of CCAAT/enhancer binding proteins $\beta$ and- $\delta$ in preadipocytes. Mol. Cell. Endocr., 15: 2037-2049.

6. Brugiapaglia, A., C. Lussiana, and G. Destefanis. 2014. Fatty acid profile and cholesterol content of beef at retail of Piemontese, Limousin and Friesian breeds. Meat Sci., 96: 568-573.

7. Calkins, C. R., T. R. Dutson, G. C. Smith, Z. L. Carpenter, and G. W. Davis. 1981. Relationship of fiber type composition to marbling and tenderness of bovine muscle. J. Food Sci., 46: 708-710.

8. Chung, K. Y., D. K. Lunt, C. B.Choi, S. H. Chae, R. D. Rhoades, T. H. Adams, B. Booren, and S. B. Smith. 2006. Lipid characteristics of subcutaneous adipose tissue and M. longissimus thoracis of Angus and Wagyu steers fed to US and Japanese endpoints. Meat Sci., 73: 432-441.

9. Coleman, R. A., and D. P. Lee. 2004. Enzymes of triacylglycerol synthesis and their regulation. Prog. Lipid Res., 43: 134-176. 
10. Darimont, C., G. Vassaux, G. Ailhaud, and R. Negrel. 1994. Differentiation of preadipose cells: paracrine role of prostacyclin upon stimulation of adipose cells by angiotensin-II. Endocrinology, 135: 2030-2036.

11. De Jager, N., N. J. Hudson, A. Reverter, R. Barnard, L. M. Cafe, P. L. Greenwood, and B. P. Dalrymple. 2013. Gene expression phenotypes for lipid metabolism and intramuscular fat in skeletal muscle of cattle. J. Anim. Sci., 91: 1112-1128.

12. Duarte, M. S., P. V. R. Paulino, A. K. Das, S. Wei, N. V. L. Serao, X. Fu, S. M. Harris, M. V. Dodson, and M. Du. 2013. Enhancement of adipogenesis and fibrogenesis in skeletal muscle of Wagyu compared with Angus cattle. J. Anim. Sci., 91: 2938-2946.

13. Fu, X., Q. Yang, B. Wang, J. Zhao, M. Zhu, S. M. Parish, and M. Du. 2017. Reduced satellite cell density and myogenesis in Wagyu compared with Angus cattle as a possible explanation of its high marbling. Animal, 12: 990-997.

14. Garin-Shkolnik, T., A. Rudich, G. S. Hotamisligil, and M. Rubinstein. 2014. FABP4 attenuates PPARY and adipogenesis and is inversely correlated with PPARY in adipose tissues. Diabetes, 63: 900-911.

15. Gorocica-Buenfil, M. A., F. L. Fluharty, C. K. Reynolds, and S. C. Loerch. 2007. Effect of dietary vitamin A concentration and roasted soybean inclusion on marbling, adipose cellularity, and fatty acid composition of beef. J. Anim. Sci., 85: 2230-2242.

16. Gotoh, T. 2003. Histochemical properties of skeletal muscles in Japanese cattle and their meat production ability. Anim. Sci. J. 74: 339-354.

17. Gotoh, T., E. Albrecht, F. Teuscher, K. Kawabata, K. Sakashita, H. Iwamoto, and J. Wegner. 2009. Differences in muscle and fat accretion in Japanese Black and European cattle. Meat Sci., 82: 300308.

18. Graham, J. F., J. Byron, A. J. Clark, G. Kearney, B. Orchard. 2009. Effect of postweaning growth and bulls selected for extremes in retail beef yield and intramuscular fat on progeny liveweight and carcass traits. Anim. Prod. Sci., 49: 493-503.

19. Gupta, R. K., Z. Arany, P. Seale, R. J. Mepani, L. Ye, H. M. Conroe, Y. A. Roby, H. Kulaga, R. R. Reed, and B. M. Spiegelman. 2010. Transcriptional control of preadipocyte determination by Zfp423. Nature, 464: 619-625.

20. Hansen, J. B., H. Zhang, T. H. Rasmussen, R. K. Petersen, E. N. Flindt, E. N., and K. Kristiansen. 2001. Peroxisome proliferator-activated receptor $\delta$ (PPARס)-mediated regulation of preadipocyte proliferation and gene expression is dependent on cAMP signaling. J. Biol. Chem., 276: 3175-3182.

21. Hausman, G. J., and R. L. Richardson. 2004. Adipose tissue angiogenesis. J. Anim. Sci., 82: 925-934.

22. Holst, D., S. Luquet, V. Nogueira, K. Kristiansen, X. Leverve, and P. A. Grimaldi. 2003. Nutritional regulation and role of peroxisome proliferator-activated receptor $\delta$ in fatty acid catabolism in skeletal muscle. Biochim. Biophys. Acta Mol. Cell Biol. Lipids, 1633: 43-50.

23. Hood, R., and C. E. Allen. 1973. Cellularity of bovine adipose tissue. J. Lipid Res., 14: 605-610. 24. Hudak, C. S., and H. S. Sul. 2013. Pref-1, a gatekeeper of adipogenesis. Front. Endo., 4: 1-6. 
25. Huang, Y., A. K. Das, Q. Y. Yang, M. J. Zhu, and M. Du. 2012. Zfp423 promotes adipogenic differentiation of bovine stromal vascular cells. PloS One, 7: e47496.

26. Hwang, Y. H., G. D. Kim, J. Y. Jeong, S. J. Hur, and S. T. Joo. 2010. The relationship between muscle fiber characteristics and meat quality traits of highly marbled Hanwoo (Korean native cattle) steers. Meat Sci., 86: 456-461.

27. Jaborek, J. R., F. L. Fluharty, H. N. Zerby, and A. E. Relling. 2021. Comparison of feedlot performance, carcass characteristics, and fatty acid composition of Angus- and Wagyu-sired cattle when raised to a similar age or body weight. Appl. Anim. Sci. Submitted.

28. Jeong, J., E. G. Kwon, S. K. Im, K. S. Seo, and M. Baik. 2012. Expression of fat deposition and fat removal genes is associated with intramuscular fat content in longissimus dorsi muscle of Korean cattle steers. J. Anim. Sci., 90: 2044-2053.

29. Jurie, C., I. Cassar-Malek, M. Bonnet, C. Leroux, D. Bauchart, P. Boulesteix, D. W. Pethick, and J. F. Hocquette. 2007. Adipocyte fatty acid-binding protein and mitochondrial enzyme activities in muscles as relevant indicators of marbling in cattle. J. Anim. Sci., 85: 2660-2669.

30. Kazala, E. C., J. L. Petrak, F. J. Lozeman, P. S. Mir, A. Laroche, J. Deng, and R. J. Weselake. 2003. Hormone-sensitive lipase activity in relation to fat content of muscle in Wagyu hybrid cattle. Livest. Prod. Sci., 79: 87-96.

31. Lancaster, P. A., E. D. Sharman, G. W. Horn, C. R. Krehbiel, and J. D. Starkey. 2014. Effect of rate of weight gain of steers during the stocker phase. III. Gene expression of adipose tissues and skeletal muscle in growing-finishing beef cattle. J. Anim. Sci., 92: 1462-1472.

32. Lunt, D. K., K. Y. Chung, C. B. Choi, and S. B. Smith. 2005. Production characteristics and carcass quality of Angus and Wagyu steers fed to US and Japanese endpoints. J. Anim. Vet. Adv., 4, 949-953.

33. Lunt, D. K., R. R., Riley, and S. B. Smith. 1993. Growth and carcass characteristics of Angus and American Wagyu steers. Meat Sci., 34, 327-334.

34. Killinger, K. M., C. R. Calkins, W. J. Umberger, D. M. Feuz, and K. M. Eskridge. 2004. Consumer sensory acceptance and value for beef steaks of similar tenderness, but differing in marbling level. J. Anim. Sci., 82, 3294-3301.

35. Kim, W. S., H. S. Park, and J. H. Sung. 2015. The pivotal role of PDGF and its receptor isoforms in adipose-derived stem cells. Histol. Histopathol., 30: 793-799.

36. Kruk, Z. A., M. J. Bottema, L. Reyes-Veliz, R. E. A. Forder, W. S. Pitchford, and C. D. Bottema. 2018. Vitamin A and marbling attributes: Intramuscular fat hyperplasia effects in cattle. Meat Sci., 137: 139-146.

37. May, S. G., H. G. Dolezal, D. R. Gill, F. K. Ray, and D. S. Buchanan. 1992. Effect of days fed, carcass grade traits, and subcutaneous fat removal on postmortem muscle characteristics and beef palatability. J. Anim. Sci., 70, 444-453.

38. May, S. G., J. W. Savell, D. K. Lunt, J. J. Wilson, J. C. Laurenz, and S. B. Smith. 1994. Evidence for preadipocyte proliferation during culture of subcutaneous and intramuscular adipose tissues from Angus and Wagyu crossbred steers. J. Anim. Sci., 72: 3110-3117. 
39. McGarry, J. D. and Brown, N. F. 1997. The mitochondrial carnitine palmitoyltransferase system: from concept to molecular analysis. Eur. J. Biochem., 244, 1-14.

40. McIntyre, T. M., A. V. Pontsler, A. R. Silva, A. S. Hilaire, Y. Xu, J. C. Hinshaw, G. A. Zimmerman, K. Hama, J. Aoki, H. Ara, and G. D. Prestwich. 2003. Identification of an intracellular receptor for lysophosphatidic acid (LPA): LPA is a transcellular PPARy agonist. Proceed. Nat. Acad. Sci., 100: 131-136.

41. McKiernan, W. A., J. F. Wilkins, J. Irwin, B. Orchard, and S. A. Barwick. 2009. Performance of steer progeny of sires differing in genetic potential for fatness and meat yield following postweaning growth at different rates. 2. Carcass traits. Anim. Prod. Sci. 49: 525-534.

42. Moisá, S. J., D. W. Shike, D. B. Faulkner, W. T. Meteer, D. Keisler, and J. J. Loor. 2014. Central role of the PPARY gene network in coordinating beef cattle intramuscular adipogenesis in response to weaning age and nutrition. Gene Reg. Sys. Biol., 8: 17-32.

43. Moore, K. K., P. A. Ekeren, D. K. Lunt, and S. B. Smith. 1991. Relationship between fatty acid-binding protein activity and marbling scores in bovine longissimus muscle. J. Anim. Sci., 69: 1515-1521.

44. Ozawa, S., T. Mitsuhashi, M. Mitsumoto, S. Matsumoto, N. Itoh, K. Itagaki, Y. Kohno, and T. Dohgo. 2000. The characteristics of muscle fiber types of longissimus thoracis muscle and their influences on the quantity and quality of meat from Japanese Black steers. Meat Sci., 54: 65-70.

45. Payne, V. A., W. S. Au, C. E. Lowe, S. M. Rahman, J. E. Friedman, S. O'Rahilly, and J. J. Rochford. 2010. C/EBP transcription factors regulate SREBP1c gene expression during adipogenesis. Biochem. J., 425: 215-224.

46. Pickworth, C. L., S. C. Loerch, S. G. Velleman, J. L. Pate, D. H. Poole, and F. L. Fluharty. 2011. Adipogenic differentiation state-specific gene expression as related to bovine carcass adiposity. J. Anim. Sci., 89: 355-366.

47. Pickworth, C. L., S. C. Loerch, and F. L. Fluharty. 2012. Restriction of vitamin A and D in beef cattle finishing diets on feedlot performance and adipose accretion. J. Anim. Sci., 90: 1866-1878.

48. Platter, W. J., J. D. Tatum, K. E. Belk, P. L. Chapman, J. A. Scanga, and G. C. Smith. 2003. Relationships of consumer sensory ratings, marbling score, and shear force value to consumer acceptance of beef strip loin steaks. J. Anim. Sci., 81, 2741-2750.

49. Rahman, M. S. 2019. Prostacyclin: A major prostaglandin in the regulation of adipose tissue development. J. Cell. Phys., 234: 3254-3262.

50. Rahman, M. S., F. Khan, P. K. Syeda, K. Nishimura, M. Jisaka, T. Nagaya, F. Shono, and K. Yokota. 2014. Endogenous synthesis of prostacyclin was positively regulated during the maturation phase of cultured adipocytes. Cytotechnology, 66: 635-646.

51. Reusch, J. E., L. A. Colton, and D. J. Klemm. 2000. CREB activation induces adipogenesis in 3WA-MDL1 cells. Mole. Cell. Biol., 20: 1008-1020.

52. Rhee, K. S., T. R. Dutson, G. C. Smith, R. L. Hostetler, and R. Reiser. 1982. Cholesterol content of raw and cooked beef longissimus muscles with different degrees of marbling. J. Food Sci., 47: 716-719. 
53. Robelin, J. 1981. Cellularity of bovine adipose tissues: developmental changes from 15 to 65 percent mature weight. J. Lipid Res., 22: 452-457.

54. Roberts, S. L., P. A. Lancaster, U. DeSilva, G. W. Horn, and C. R. Krehbiel. 2015. Coordinated gene expression between skeletal muscle and intramuscular adipose tissue in growing beef cattle. J. Anim. Sci., 93: 4302-4311.

55. Rule, D. C., M. D. MacNeil, and R. E. Short. 1997. Influence of sire growth potential, time on feed, and growing-finishing strategy on cholesterol and fatty acids of the ground carcass and longissimus muscle of beef steers. J. Anim. Sci., 75: 1525-1533.

56. Schiavetta, A. M., M. F. Miller, D. K. Lunt, S. K. Davis, and S. B. Smith. 1990. Adipose tissue cellularity and muscle growth in young steers fed the beta-adrenergic agonist clenbuterol for 50 days and after 78 days of withdrawal. J. Anim. Sci., 68: 3614-3623.

57. Schoonmaker, J. P., F. L. Fluharty, and S. C. Loerch. 2004. Effect of source and amount of energy and rate of growth in the growing phase on adipocyte cellularity and lipogenic enzyme activity in the intramuscular and subcutaneous fat depots of Holstein steers. J. Anim. Sci., 82: 137-148.

58. Shimano, H. 2001. Sterol regulatory element-binding proteins (SREBPs): transcriptional regulators of lipid synthetic genes. Prog. Lipid Res., 40: 439-452.

59. Tang, Q. Q., T. C. Otto, and M. D. Lane. 2003. CCAAT/enhancer-binding protein $\beta$ is required for mitotic clonal expansion during adipogenesis. Proc. Nat. Acad. Sci., 100: 850-855.

60. Tontonoz, P., E. Hu, R. A. Graves, A. I. Budavari, and B. M. Spiegelman. 1994. mPPAR gamma 2: tissue-specific regulator of an adipocyte enhancer. Genes \& Devel., 8: 1224-1234.

61. Uezumi, A., S. I. Fukada, N. Yamamoto, S. I. Takeda, K. Tsuchida. 2010. Mesenchymal progenitors distinct from satellite cells contribute to ectopic fat cell formation in skeletal muscle. Nat. Cell Biol., 12: 143-153.

62. Underwood, K. R., J. Tong, M. J. Zhu, Q. W. Shen, W. J. Means, S. P. Ford, S. I. Paisley, B. W. Hess, and M. Du. 2007. Relationship between kinase phosphorylation, muscle fiber typing, and glycogen accumulation in longissimus muscle of beef cattle with high and low intramuscular fat. J. Agric. Food Chem., 55: 9698-9703.

63. Waddell J. N., P. Zhang, Y. Wen, S. K. Gupta, A. Yevtodiyenko, J. V. Schmidt, C. A. Bidwell, A. Kumar, and S. Kuang. 2010. Dlk1 is necessary for proper skeletal muscle development and regeneration. PLoS ONE 5: e15055.

64. Wang, Y. H., B. L. Bower, A. Reverter, S. H. Tan, N. De Jager, R. Wang, S. M. McWilliam, L. M. Cafe, P. L. Greenwood, and S. A. Lehnert. 2009. Gene expression patterns during intramuscular fat development in cattle. J. Anim. Sci., 87: 119-130.

65. Wheeler, T. L., G. W. Davis, B. J. Stoecker, and C. J. Harmon. 1987. Cholesterol concentration of longissimus muscle, subcutaneous fat and serum of two beef cattle breed types. J. Anim. Sci., 65: 1531-1537.

66. Woollett, L. A., D. K. Spady, and J. M. Dietschy. 1992. Saturated and unsaturated fatty acids independently regulate low density lipoprotein receptor activity and production rate. J. Lipid Res., 33: 
77-88.

67. Wu, Z., N. L. Bucher, and S. R. Farmer. 1996. Induction of peroxisome proliferator-activated receptor gamma during the conversion of 3WA-MD fibroblasts into adipocytes is mediated by C/EBPbeta, C/EBPdelta, and glucocorticoids. Mole. Cell. Biol., 16: 4128-4136.

68. Yamada, T., S. Kawakami, and N. Nakanishi. 2010. Fat depot-specific differences in angiogenic growth factor gene expression and its relation to adipocyte size in cattle. J. Vet. Med. Sci., 72: 991997.

69. Yamada, T., and N. Nakanishi. 2012. Effects of the roughage/concentrate ratio on the expression of angiogenic growth factors in adipose tissue of fattening Wagyu steers. Meat Sci., 90: 807-813.

70. Zhang, P., K. Takeuchi, L. S. Csaki, and K. Reue. 2012. Lipin-1 phosphatidic phosphatase activity modulates phosphatidate levels to promote peroxisome proliferator-activated receptor y (PPARY) gene expression during adipogenesis. J. Biol. Chem., 287: 3485-3494.

\section{Figures}

$1 \mathrm{~A}$

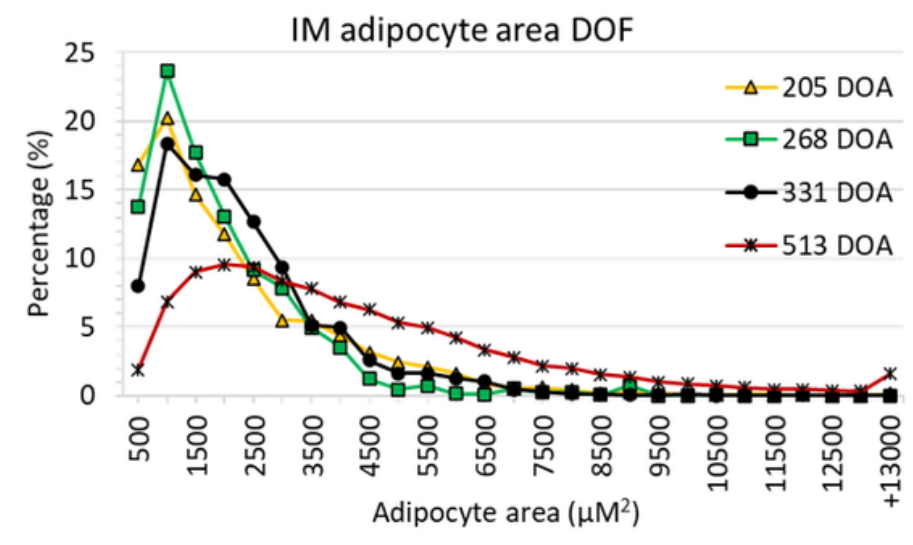

$1 \mathrm{~B}$

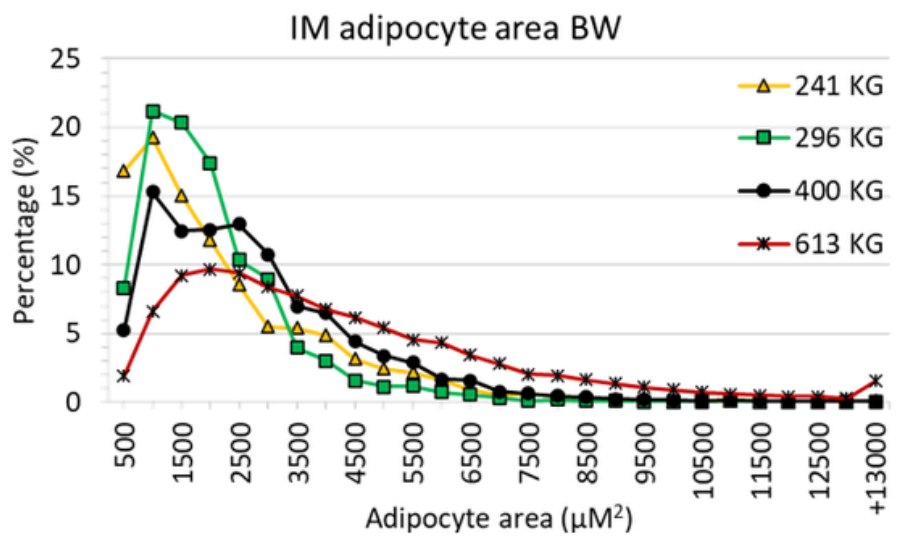

\section{Figure 1}

Distribution of intramuscular (IM) adipocyte area in the longissimus muscle of steers compared $A$ ) at a similar number of days on feed (DOF) and age $(205,268,331,422,513$ days of age) or B) at a similar

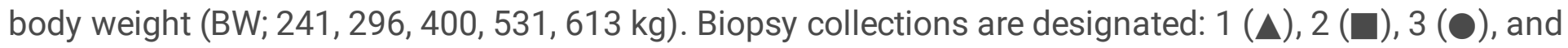
$5(Ж)$. 
A

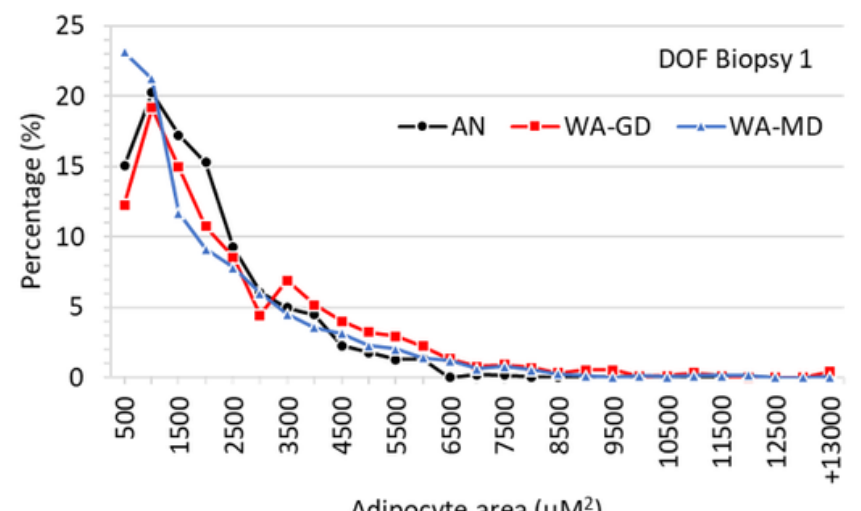

Adipocyte area $\left(\mu \mathrm{M}^{2}\right)$

C

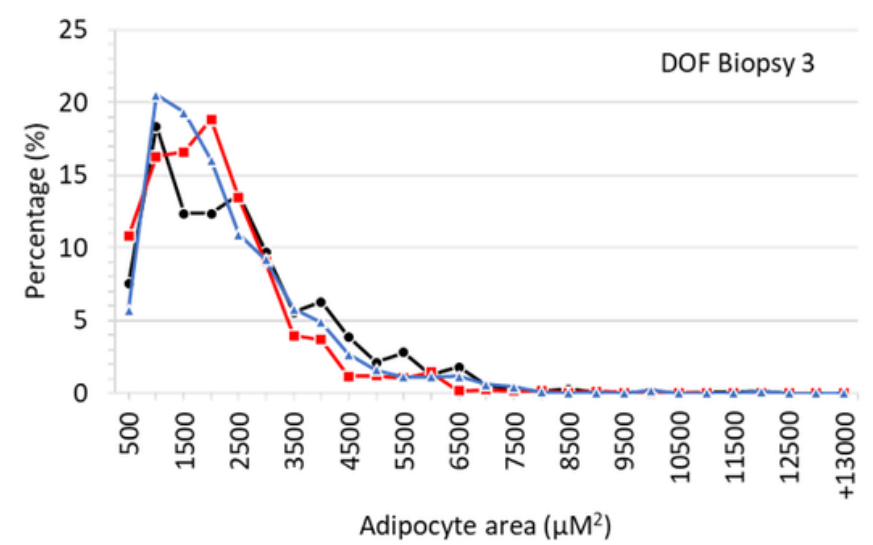

B

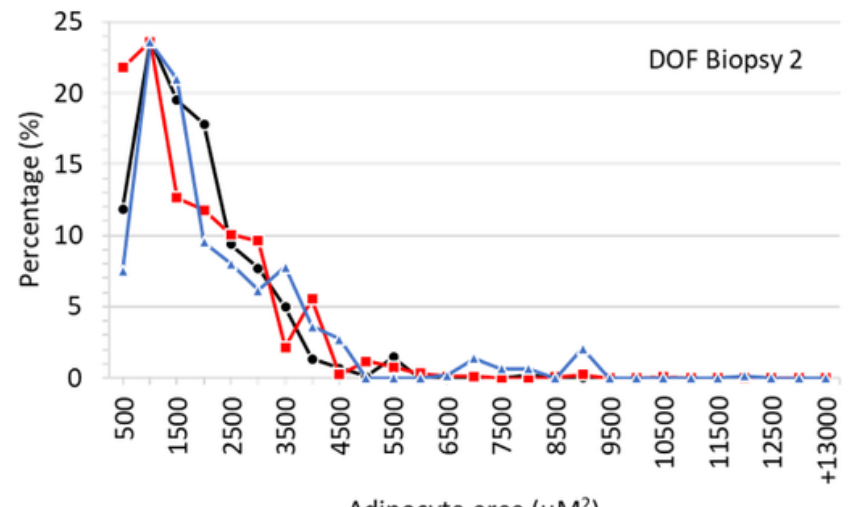

Adipocyte area $\left(\mu \mathrm{M}^{2}\right)$

D

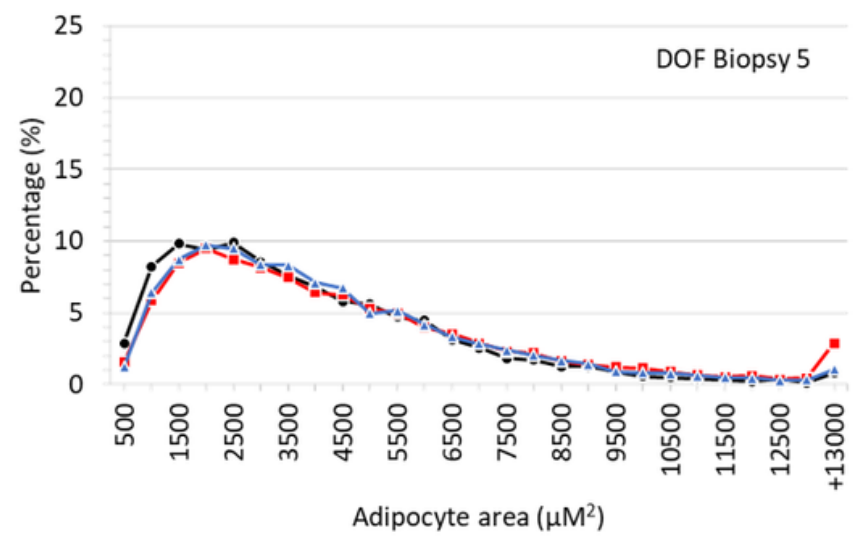

Figure 2

Distribution of intramuscular adipocyte (IM) area in the longissimus muscle of steers compared at a similar number of days on feed (DOF) and age: 205, 268, 331, and 513 days of age, for biopsy collections $1,2,3$, and 5, respectively. Treatment LSmean estimates are designated as AN ( $)$, WA-GB ( $\square$ ), and WAMB (A). 
3A

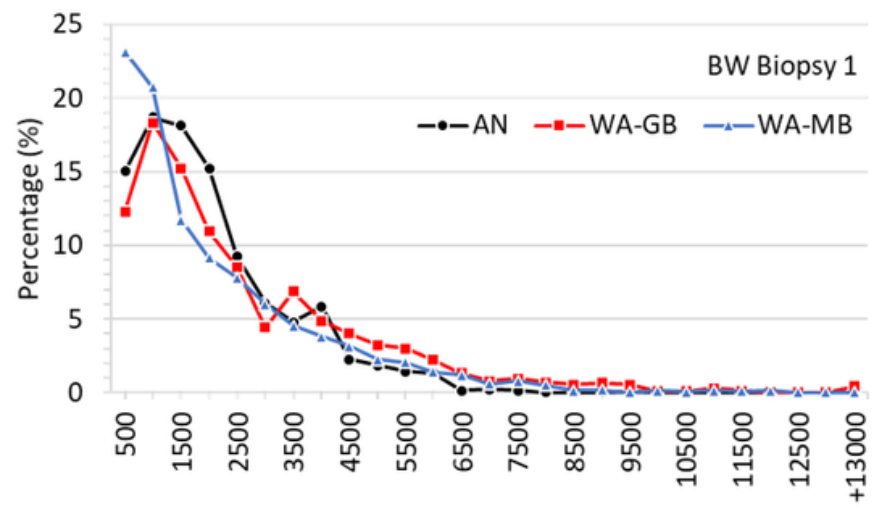

Adipocyte area ( $\mu \mathrm{M} 2)$

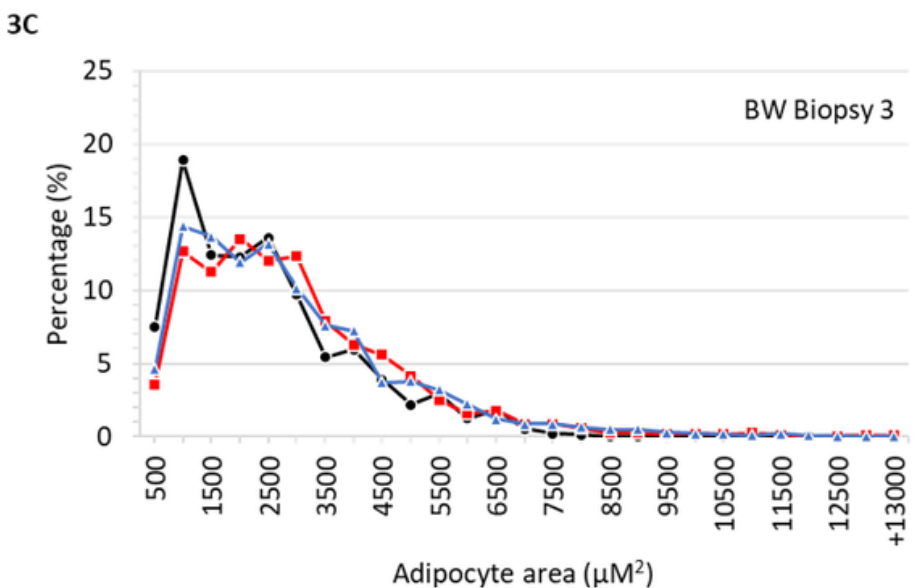

3B

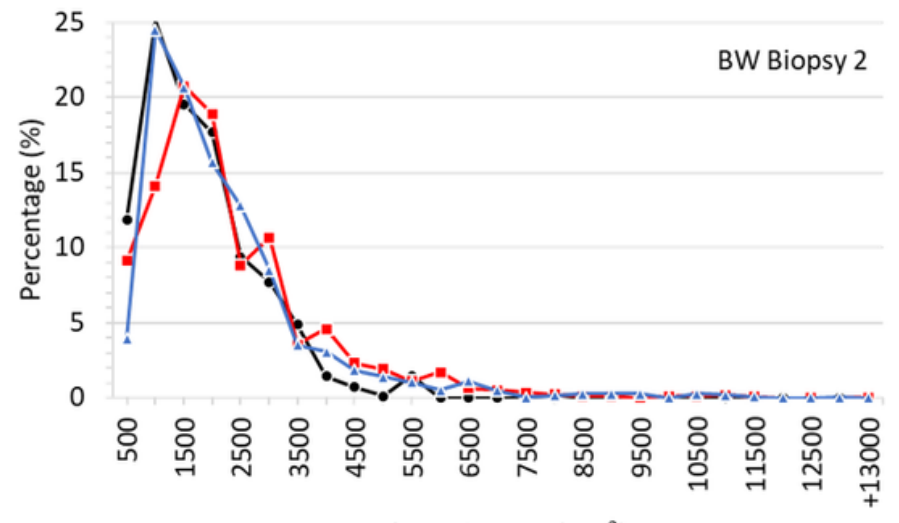

Adipocyte area $\left(\mu \mathrm{M}^{2}\right)$

3D

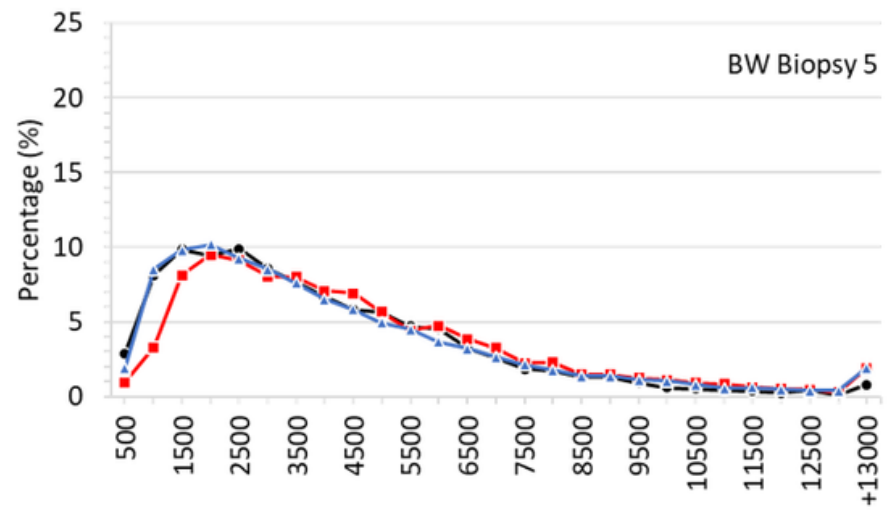

Adipocyte area $\left(\mu \mathrm{M}^{2}\right)$

\section{Figure 3}

Distribution of intramuscular adipocyte (IM) area in the longissimus muscle of steers compared at a similar body weight (BW): 241, 296, 400, and $613 \mathrm{~kg}$, for biopsy collections 1, 2, 3, and 5, respectively.

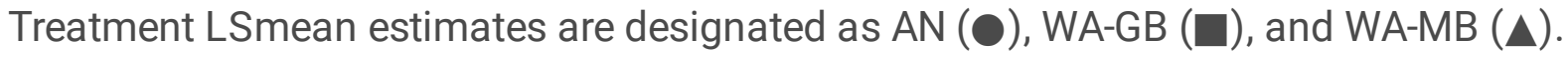

$4 \mathrm{~A}$

ZFP423 DOF

$\mathrm{TRT}=0.73 \quad \mathrm{BC}=0.01 \quad \mathrm{TRT} \times \mathrm{BC}=0.70$

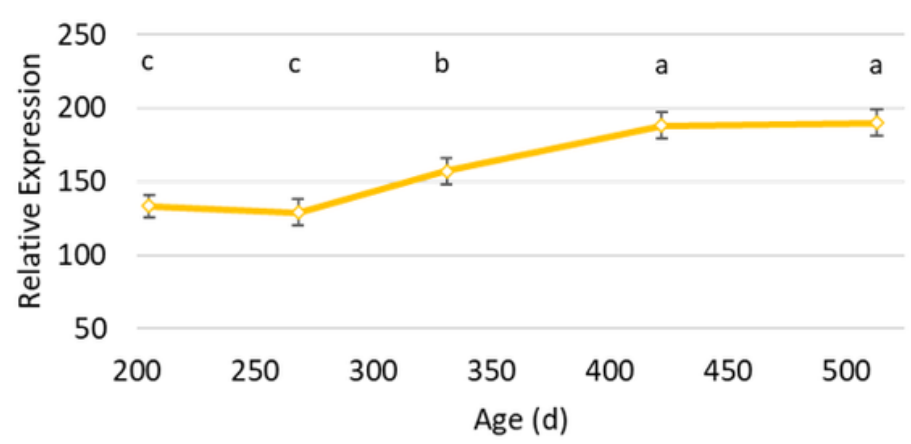

4B

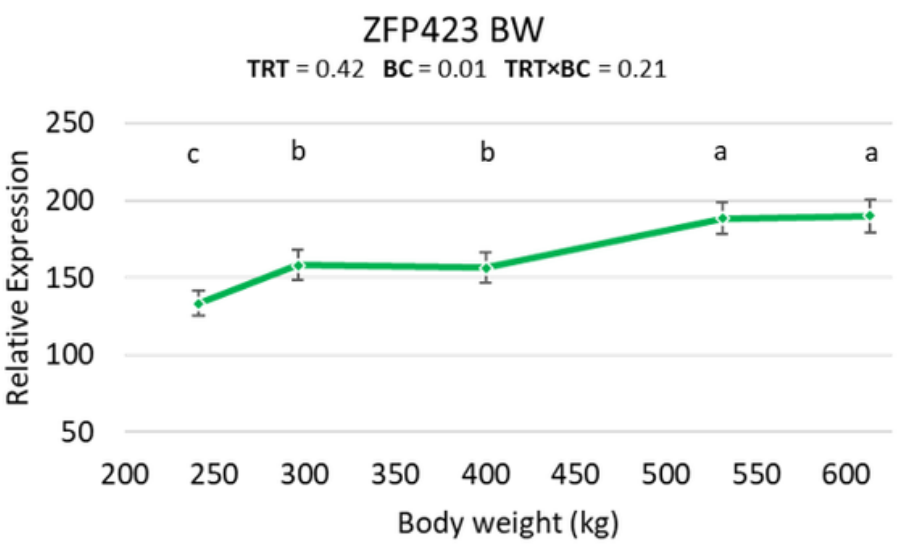

\section{Figure 4}


Expression of zinc finger protein 423 (ZFP423) in the longissimus muscle of steers compared A) at a similar number of days on feed (DOF) and age $(205,268,331,422,513$ days of age) or B) at a similar body weight (BW; 241, 296, 400, 531, 613 kg). LSmean estimates across biopsy number (BC) with different superscripts differ $(P \leq 0.05)$.

$5 A$

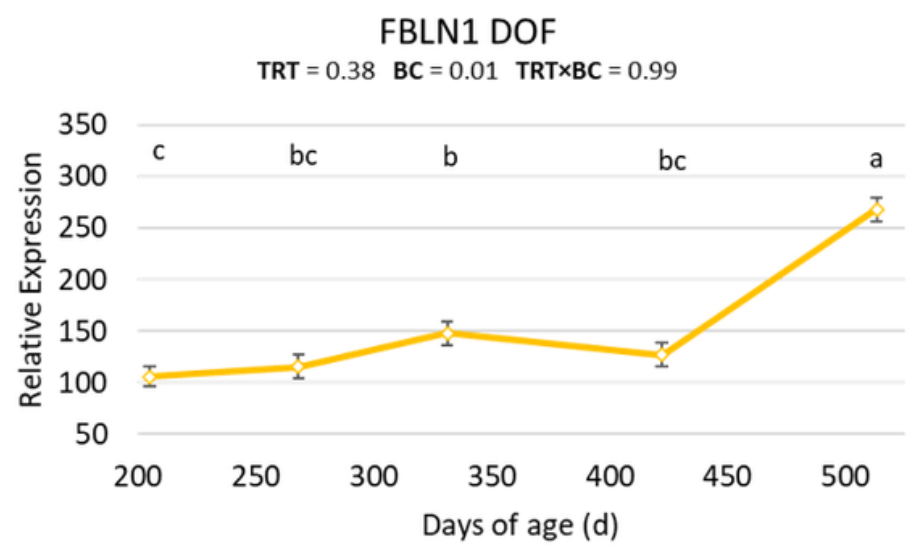

5B

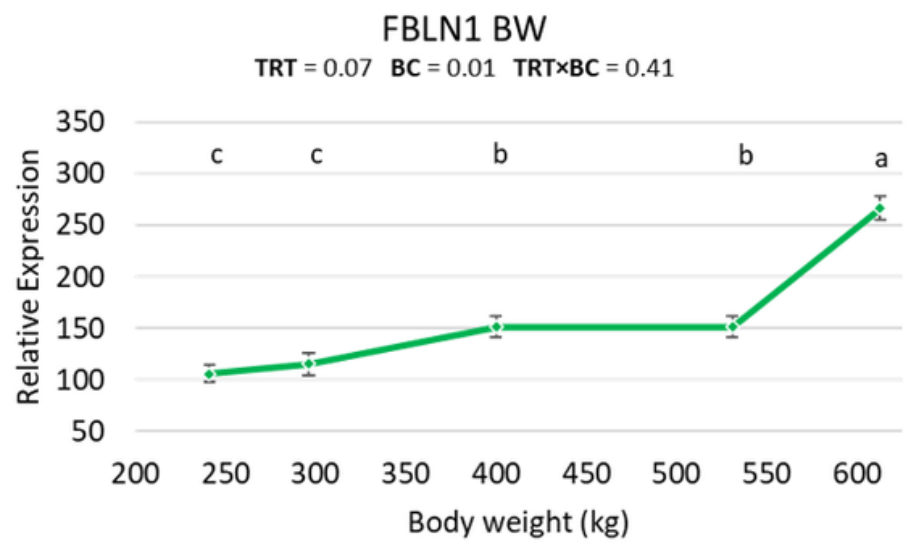

\section{Figure 5}

Expression of fibulin 1 (FBLN1) in the longissimus muscle of steers compared A) at a similar number of days on feed (DOF) and age (205, 268, 331, 422, 513 days of age) or B) at a similar body weight (BW; 241, 296, 400, 531, 613 kg). LSmean estimates across biopsy number (BC) with different superscripts differ $(P \leq 0.05)$.

$6 \mathrm{~A}$

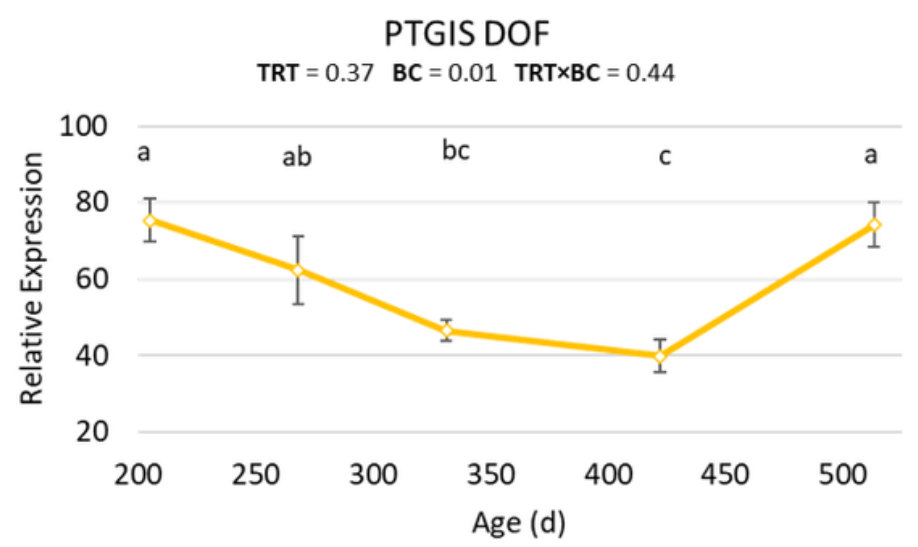

6B

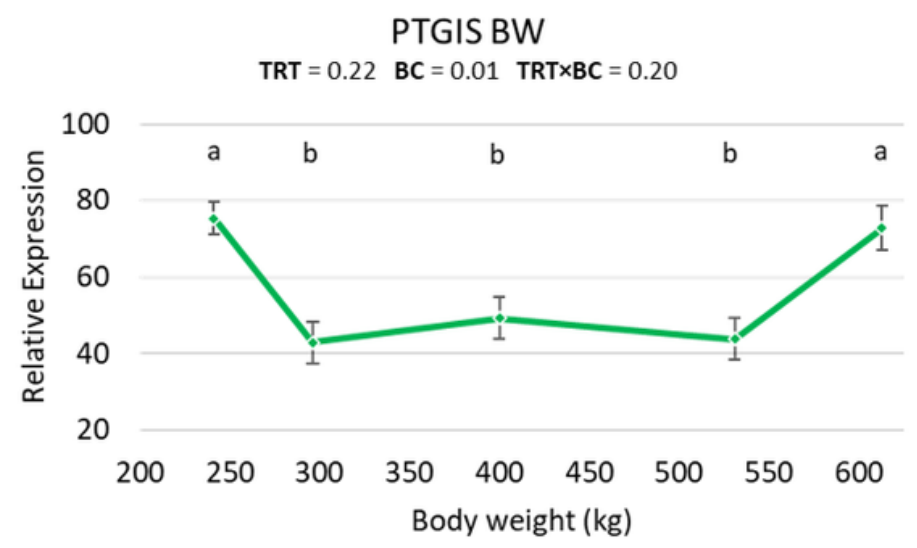

\section{Figure 6}

Expression of prostacyclin synthase (PTGIS) in the longissimus muscle of steers compared A) at a similar number of days on feed (DOF) and age $(205,268,331,422,513$ days of age) or B) at a similar body weight (BW; 241, 296, 400, 531, 613 kg). LSmean estimates across biopsy collections (BC) with different superscripts differ $(P \leq 0.05)$. 

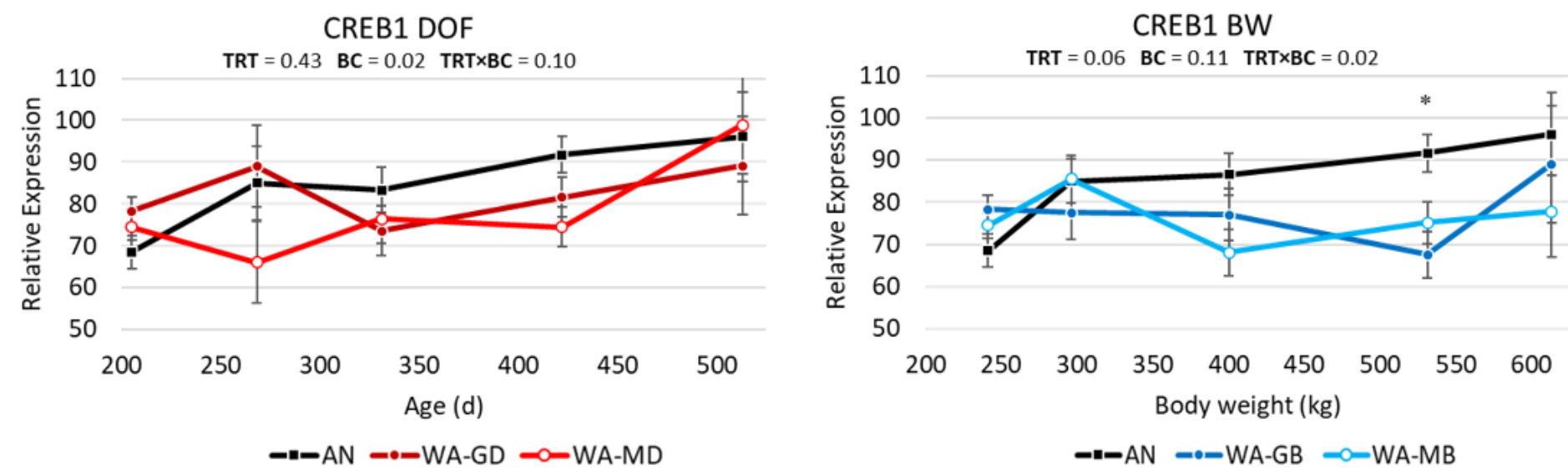

Figure 7

Expression of cAMP responsive element binding protein 1 (CREB1) in the longissimus muscle of steers compared A) at a similar number of days on feed (DOF) and age $(205,268,331,422,513$ days of age) or B) at a similar body weight (BW; 241, 296, 400,531, $613 \mathrm{~kg}$ ). LSmean estimates between AN ( $\square$ ), WA-GD $(\bullet)$, and WA-MD $(\bullet)$ steers at a given biopsy with a $\left(^{*}\right)$ differ $(P \leq 0.05)$. LSmean estimates between AN $(\square)$, WA-GB $(\bullet)$, and WA-MB $(\bullet)$ steers at a given biopsy with a $\left(^{*}\right)$ differ $(P \leq 0.05)$.

$8 \mathrm{~A}$

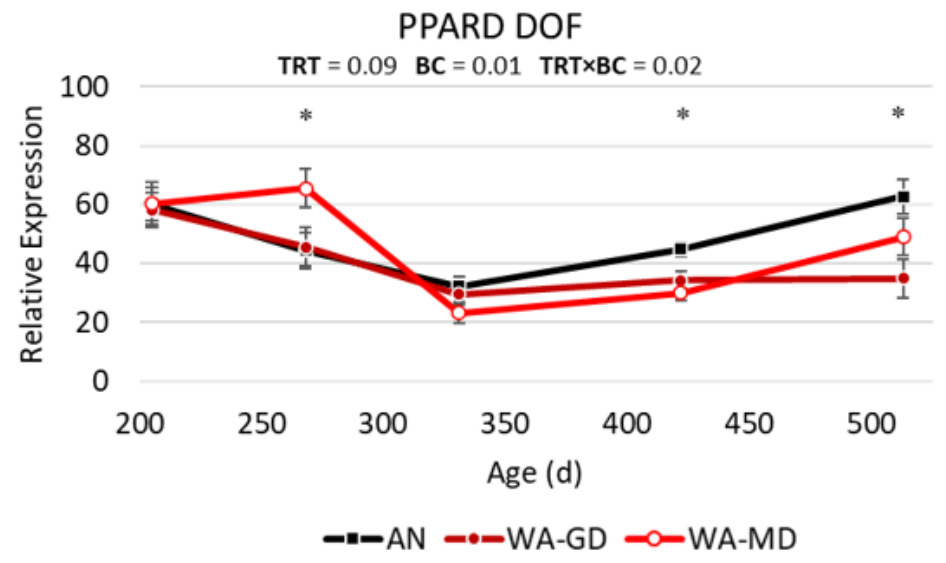

8B

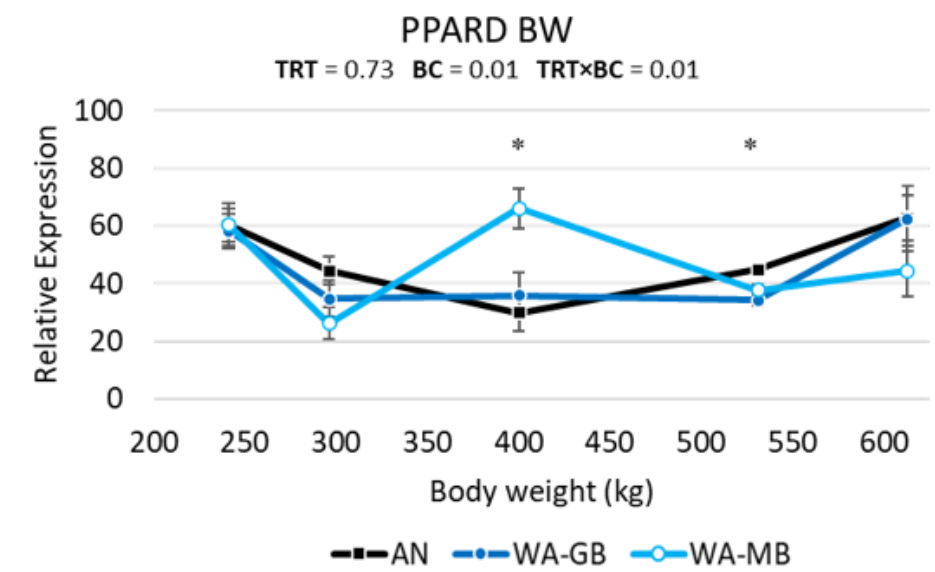

\section{Figure 8}

Expression of peroxisome proliferator activated receptor delta (PPARD) in the longissimus muscle of steers compared A) at a similar number of days on feed (DOF) and age $(205,268,331,422,513$ days of age) or B) at a similar body weight (BW; 241, 296, 400, 531, $613 \mathrm{~kg}$ ). LSmean estimates between AN ( $\square$ ), WA-GD $(\bullet)$, and WA-MD $(\bullet)$ steers at a given biopsy with a $(*)$ differ $(P \leq 0.05)$. LSmean estimates between AN $(\boldsymbol{\square})$, WA-GB $(\bullet)$, and WA-MB $(\bullet)$ steers at a given biopsy with a $(*)$ differ $(\mathrm{P} \leq 0.05)$. 
CEBPB DOF

TRT $=0.64 \quad B C=0.01 \quad T R T \times B C=0.01$

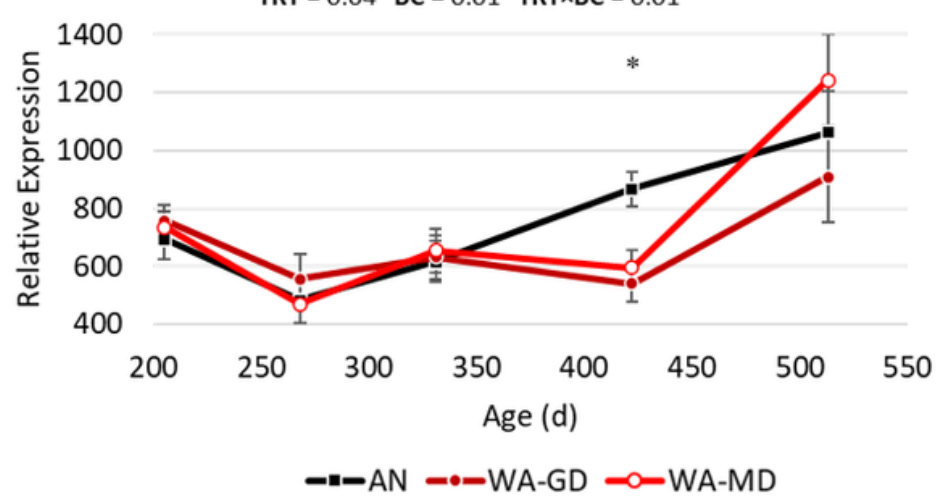

CEBPB BW

$\mathrm{TRT}=0.72 \quad \mathrm{BC}=0.01 \quad \mathrm{TRT} \times \mathrm{BC}=0.15$

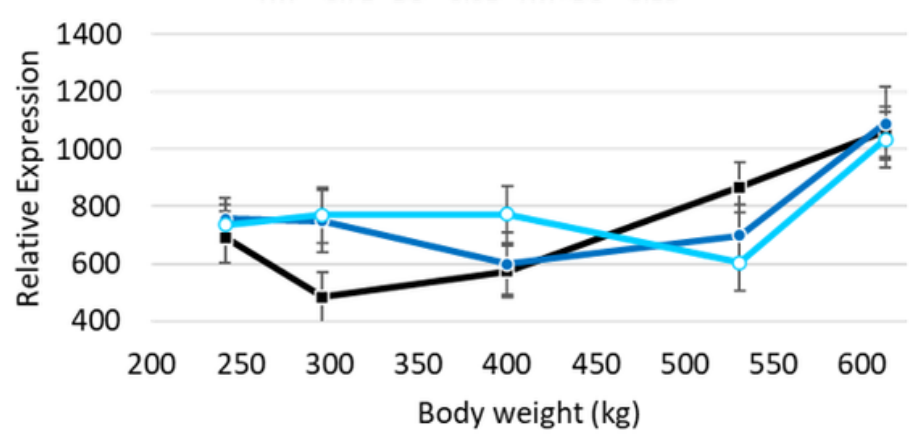

$\because-A N=W A-G B \sim W-M B$

\section{Figure 9}

Expression of CCAAT enhancer binding protein beta (CEBPB) in the longissimus muscle of steers compared A) at a similar number of days on feed (DOF) and age $(205,268,331,422,513$ days of age) or B) at a similar body weight (BW; 241, 296, 400, 531, $613 \mathrm{~kg}$ ). LSmean estimates between AN ( $\square$ ), WA-GD $(\bullet)$, and WA-MD (๑) steers at a given biopsy with a $\left({ }^{*}\right)$ differ $(P \leq 0.05)$. LSmean estimates across biopsy collections $(B C)$ with different superscripts differ $(P \leq 0.05)$.

\section{CEBPA DOF \\ TRT $=0.15 \quad B C=0.01 \quad$ TRT $\times B C=0.36$}

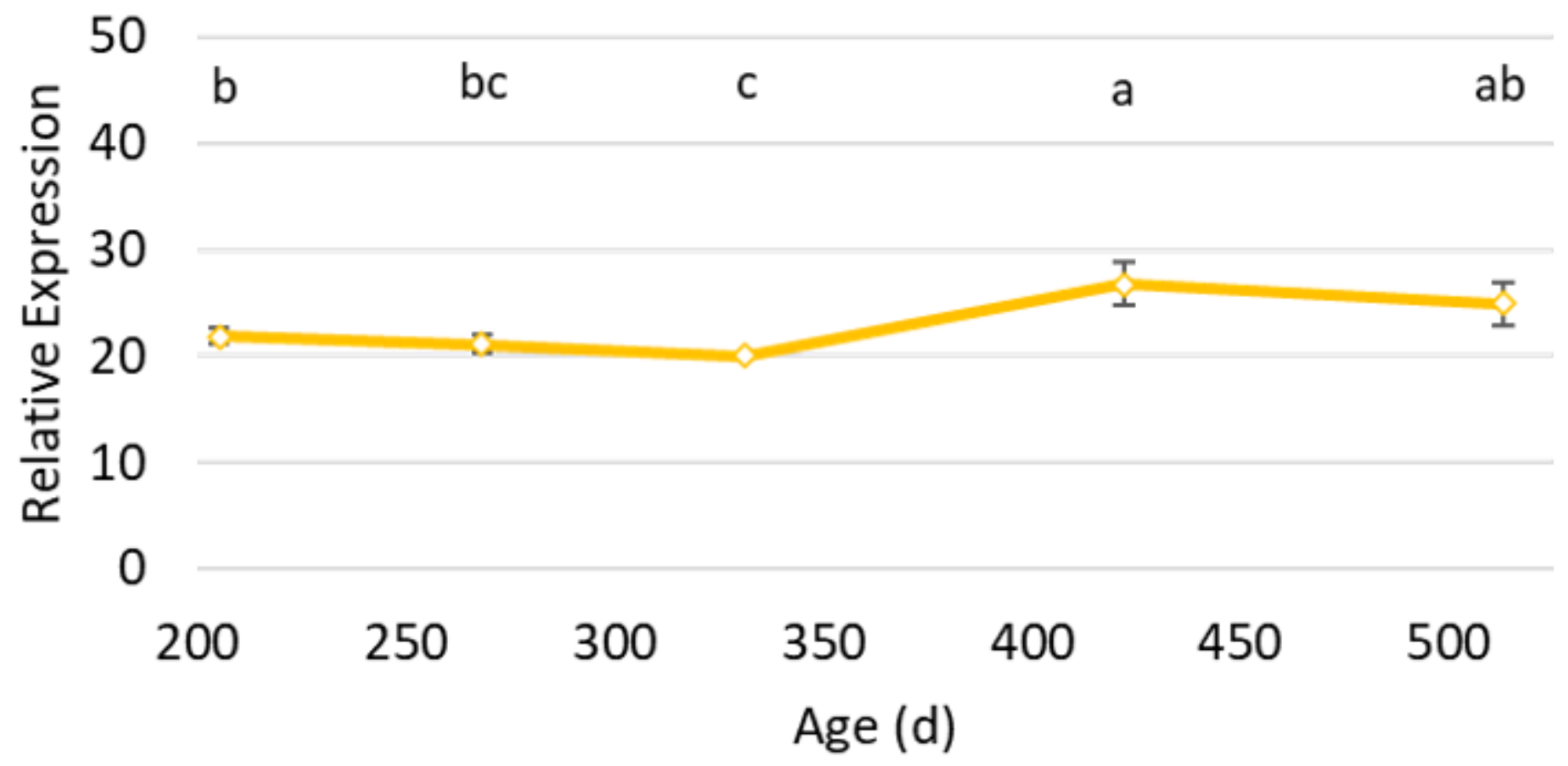

Figure 10 
Expression of CCAAT enhancer binding protein alpha (CEBPA) in the longissimus muscle of steers compared A) at a similar number of days on feed (DOF) and age $(205,268,331,422,513$ days of age) or B) at a similar body weight (BW; $241,296,400,531,613 \mathrm{~kg}$ ). LSmean estimates across biopsy collections (BC) with different superscripts differ $(P \leq 0.05)$.

$11 \mathrm{~A}$

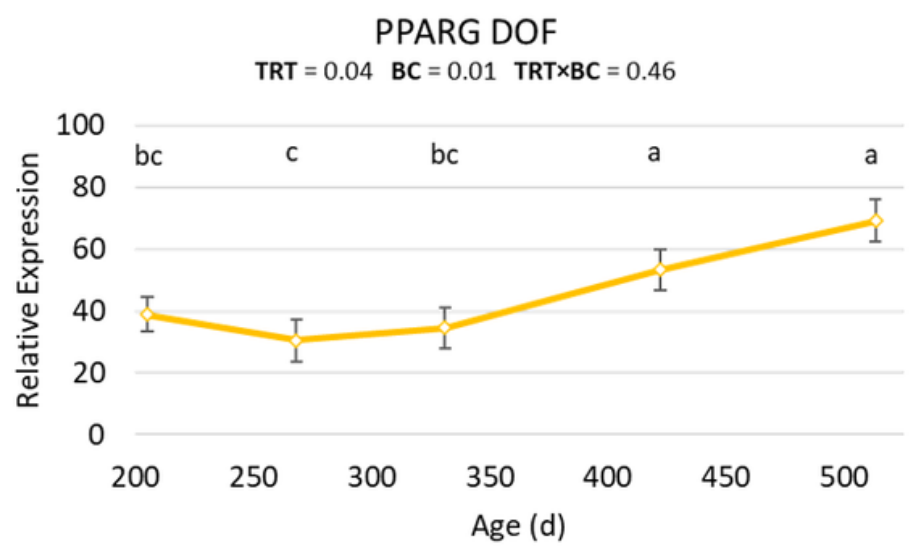

11B

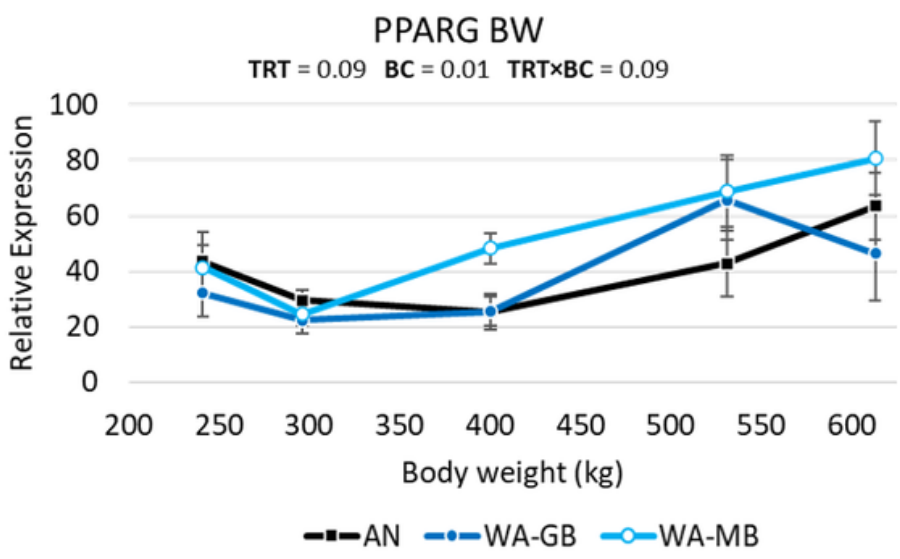

\section{Figure 11}

Expression of peroxisome proliferator activated receptor gamma (PPARG) in the longissimus muscle of steers compared $A$ ) at a similar number of days on feed (DOF) and age $(205,268,331,422,513$ days of age) or B) at a similar body weight (BW; 241, 296, 400, 531, $613 \mathrm{~kg}$ ). LSmean estimates across biopsy collections $(B C)$ with different superscripts differ $(P \leq 0.05)$. LSmean estimates between AN $(\square)$, WA-GB $(\bullet)$, and WA-MB $(\bullet)$ steers at a given biopsy with a $\left(^{*}\right)$ differ $(P \leq 0.05)$.

$12 \mathrm{~A}$

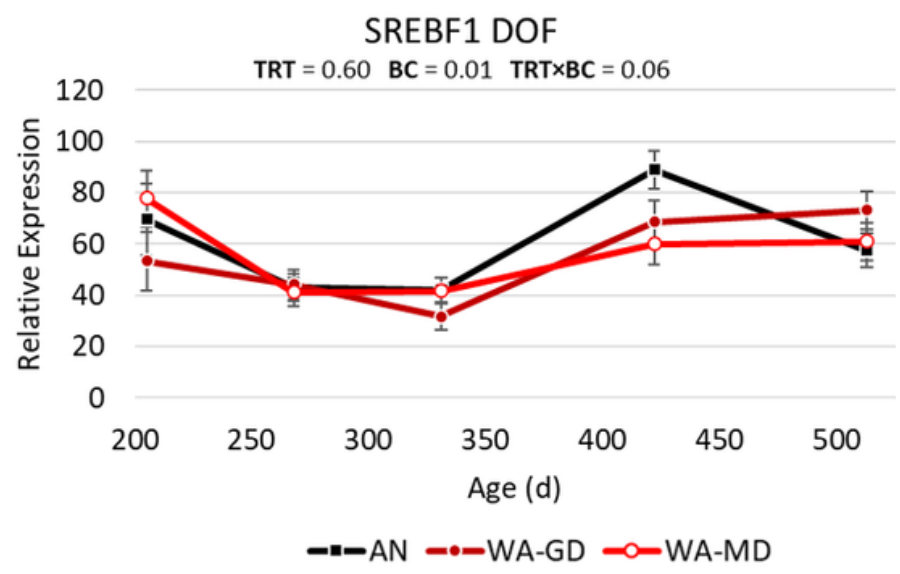

$12 \mathrm{~B}$

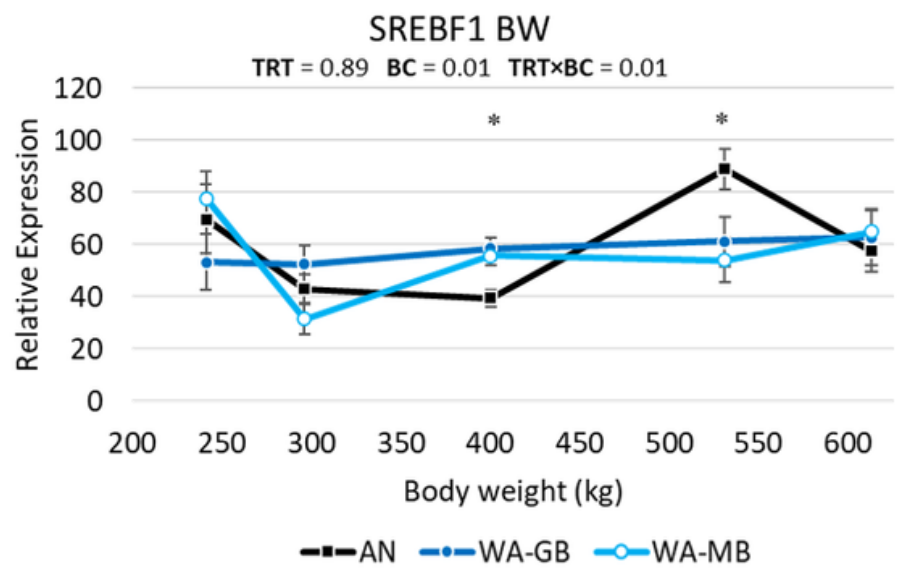

\section{Figure 12}

Expression of sterol regulatory element binding transcription factor 1 (SREBF1) in the longissimus muscle of steers compared A) at a similar number of days on feed (DOF) and age $(205,268,331,422$, 513 days of age) or B) at a similar body weight (BW; $241,296,400,531,613 \mathrm{~kg}$ ). LSmean estimates between AN $(\boldsymbol{\square})$, WA-GD $(\bullet)$, and WA-MD $(\bullet)$ steers at a given biopsy with a $\left(^{*}\right)$ differ $(P \leq 0.05)$. 
LSmean estimates between AN $(\boldsymbol{\square})$, WA-GB $(\bullet)$, and WA-MB $(\bullet)$ steers at a given biopsy with a $\left(^{(}\right)$differ $(P \leq 0.05)$.

$13 \mathrm{~A}$

ACLY DOF

$T R T=0.18 \quad B C=0.01 \quad$ TRT $\times B C=0.79$

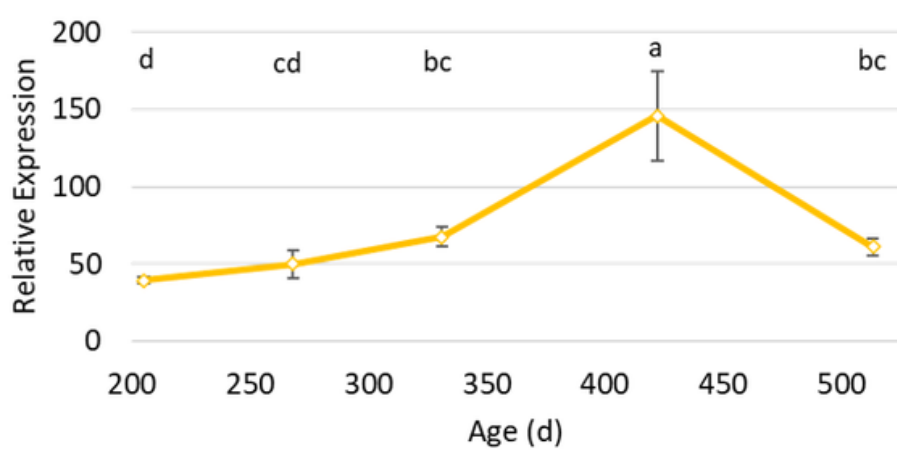

13B

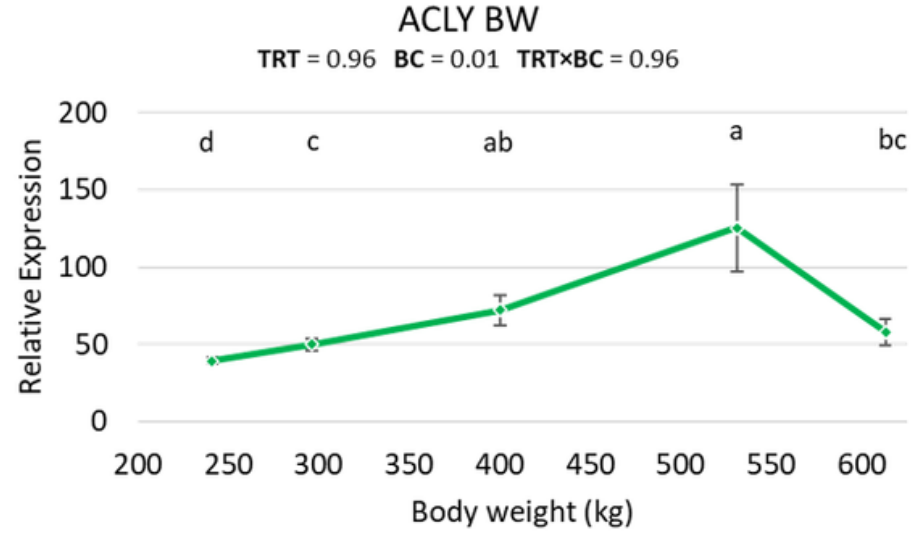

Figure 13

Expression of ATP citrate lyase (ACLY) in the longissimus muscle of steers compared A) at a similar number of days on feed (DOF) and age $(205,268,331,422,513$ days of age) or B) at a similar body weight (BW; 241, 296, 400, 531, $613 \mathrm{~kg}$ ). LSmean estimates across biopsy collections (BC) with different superscripts differ $(P \leq 0.05)$.

$14 \mathrm{~A}$

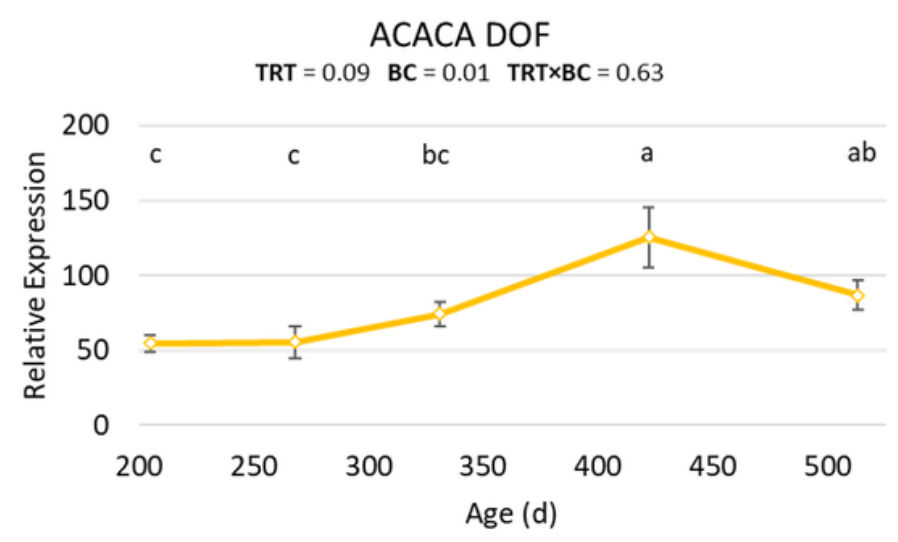

14B

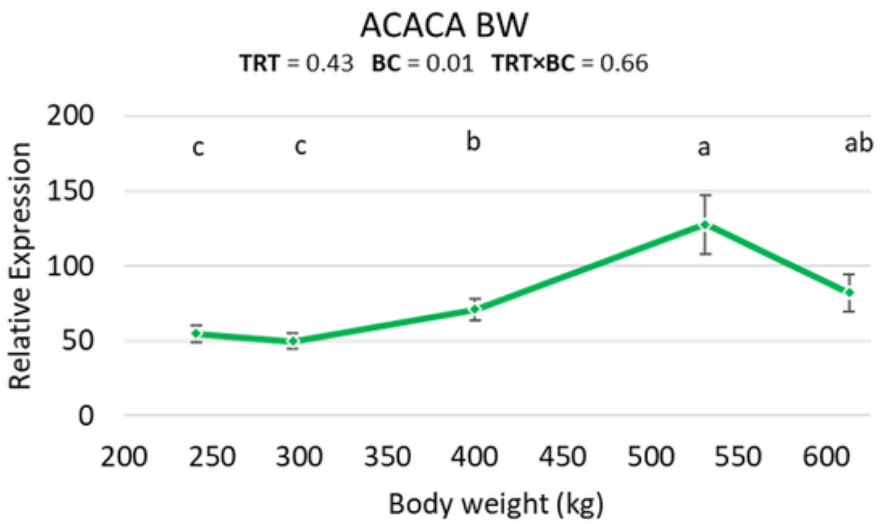

Figure 14

Expression of acetyl-CoA carboxylase 1 (ACACA) in the longissimus muscle of steers compared $A$ ) at a similar number of days on feed (DOF) and age $(205,268,331,422,513$ days of age) or B) at a similar body weight (BW; 241, 296, 400, 531, $613 \mathrm{~kg}$ ). LSmean estimates across biopsy collections (BC) with different superscripts differ $(P \leq 0.05)$. 

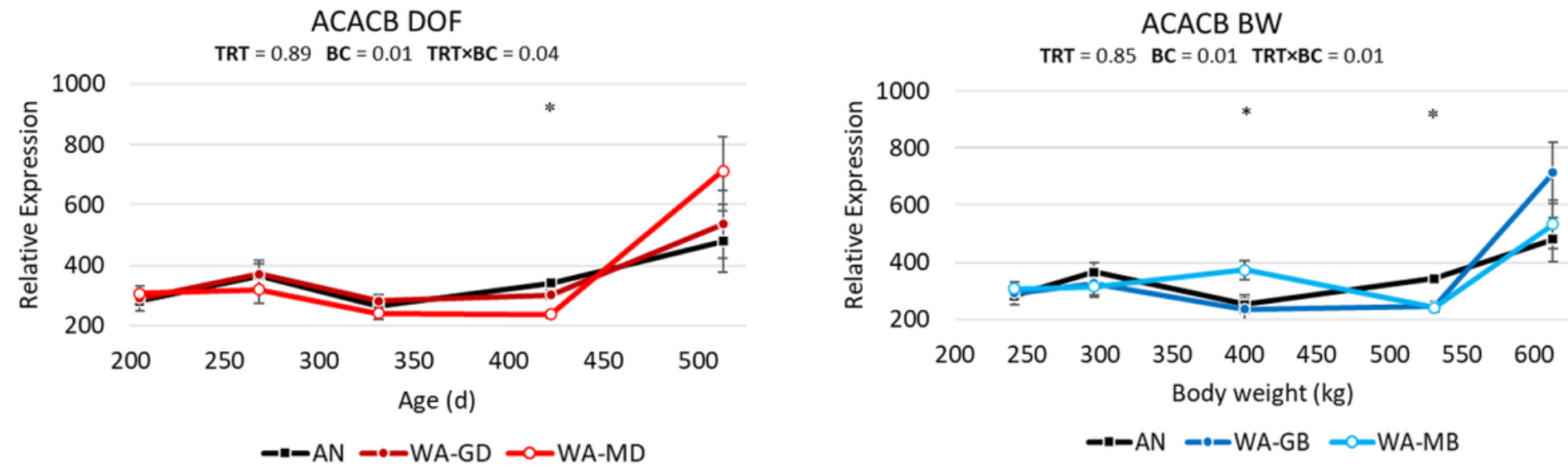

\section{Figure 15}

Expression of acetyl-CoA carboxylase 2 (ACACB) in the longissimus muscle of steers compared $A$ ) at a similar number of days on feed (DOF) and age $(205,268,331,422,513$ days of age) or B) at a similar body weight (BW; 241, 296, 400, 531, $613 \mathrm{~kg}$ ). LSmean estimates between AN (ם), WA-GD ( $)$, and WAMD (๑) steers at a given biopsy with a $\left(^{*}\right)$ differ $(P \leq 0.05)$. LSmean estimates between AN $(\square)$, WA-GB $(\bullet)$, and WA-MB $(\bullet)$ steers at a given biopsy with a $(*)$ differ $(P \leq 0.05)$.

$16 \mathrm{~A}$

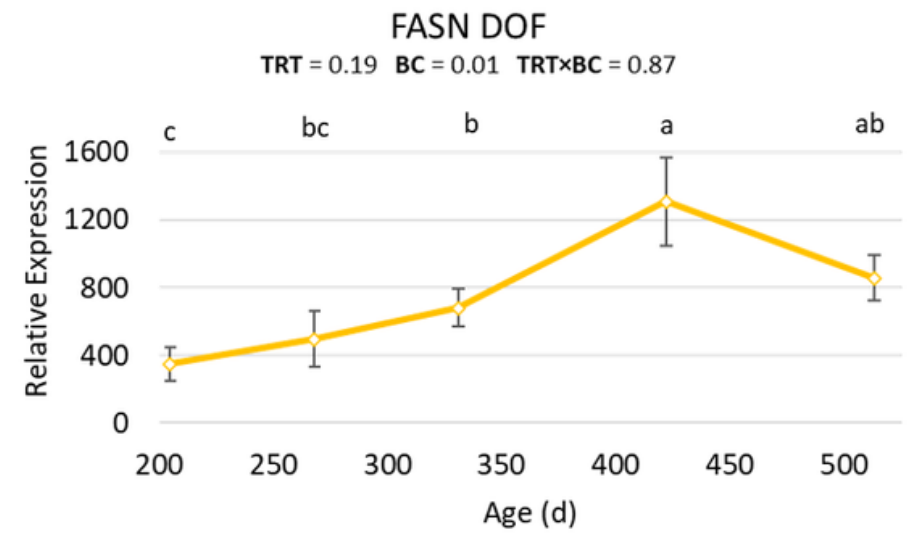

16B

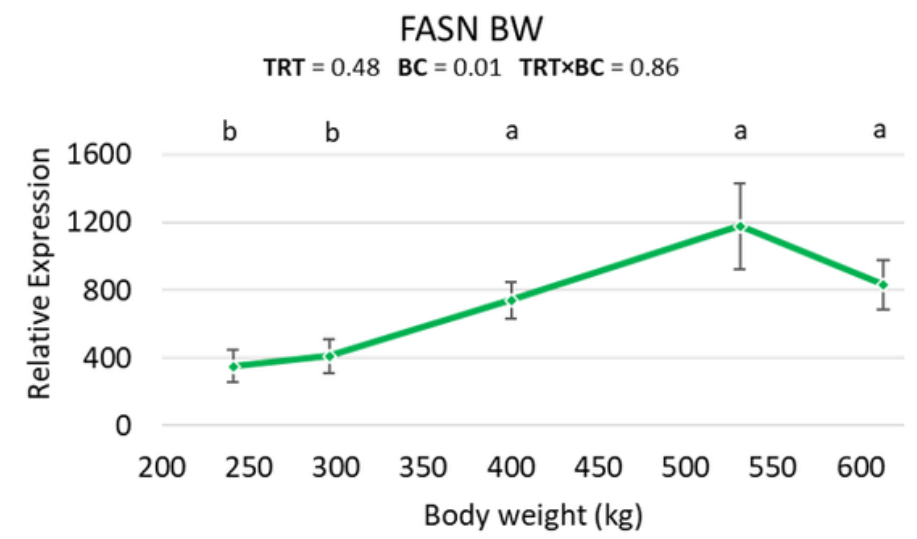

\section{Figure 16}

Expression of fatty acid synthase (FASN) in the longissimus muscle of steers compared $A$ ) at a similar number of days on feed (DOF) and age $(205,268,331,422,513$ days of age) or B) at a similar body weight (BW; 241, 296, 400, 531, 613 kg). LSmean estimates across biopsy collections (BC) with different superscripts differ $(P \leq 0.05)$. 

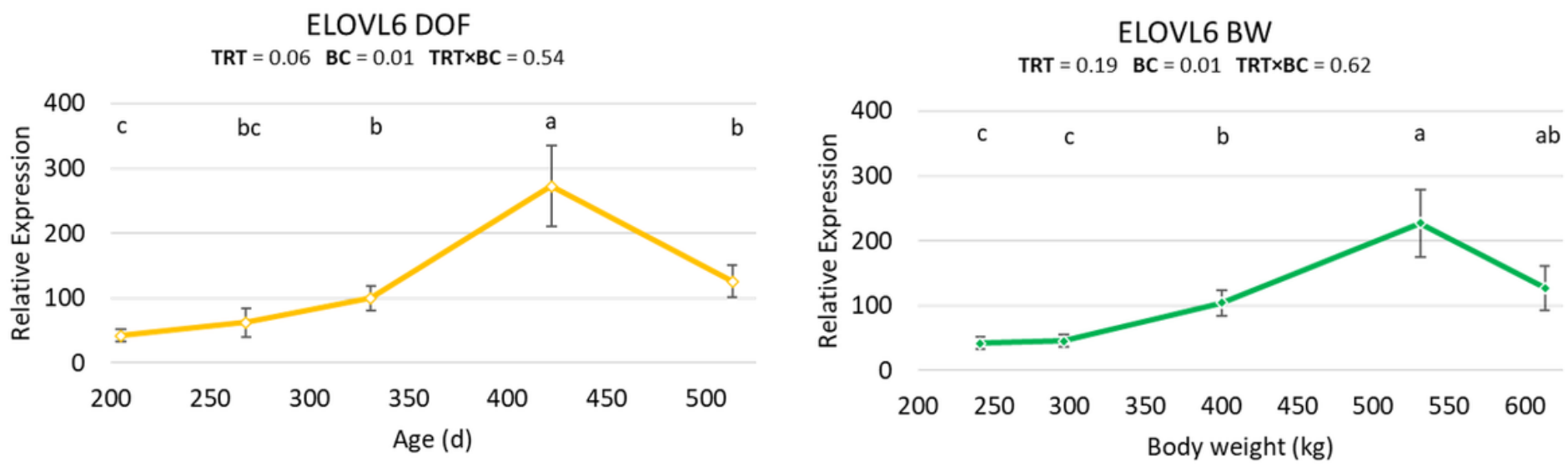

Figure 17

Expression of fatty acid elongase 6 (ELOVL6) in the longissimus muscle of steers compared A) at a similar number of days on feed (DOF) and age $(205,268,331,422,513$ days of age) or B) at a similar body weight (BW; 241, 296, 400, 531, $613 \mathrm{~kg}$ ). LSmean estimates across biopsy collections (BC) with different superscripts differ $(\mathrm{P} \leq 0.05)$.

$18 \mathrm{~A}$

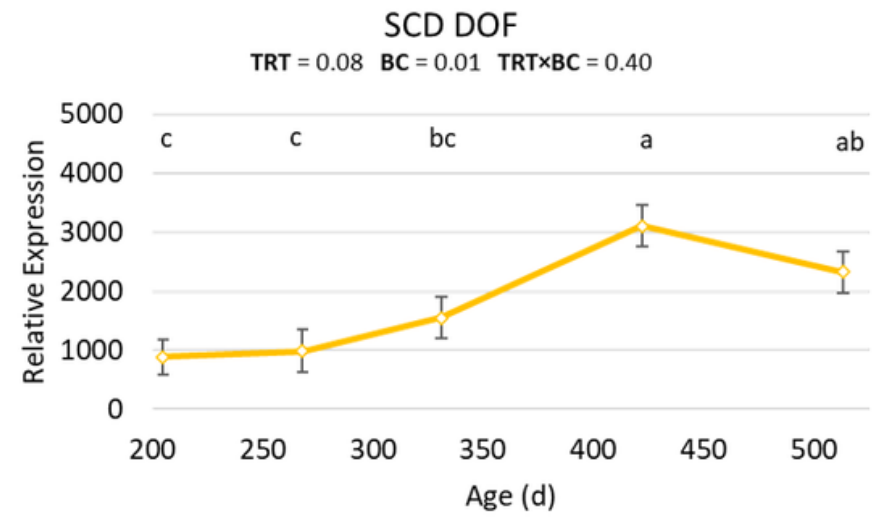

$18 \mathrm{~B}$

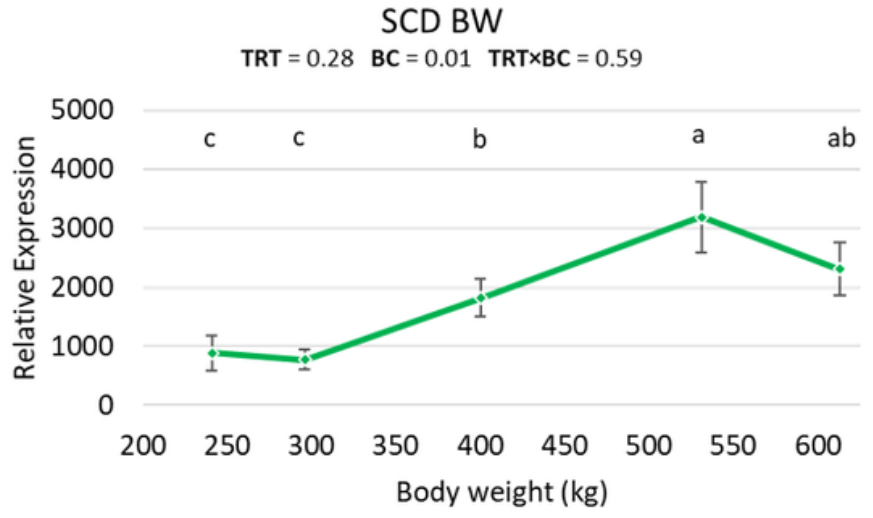

\section{Figure 18}

Expression of stearoyl-CoA desaturase (SCD) in the longissimus muscle of steers compared A) at a similar number of days on feed (DOF) and age $(205,268,331,422,513$ days of age) or B) at a similar body weight (BW; $241,296,400,531,613 \mathrm{~kg}$ ). LSmean estimates across biopsy collections (BC) with different superscripts differ $(P \leq 0.05)$. 
GPD1 DOF

$\mathrm{TRT}=0.85 \quad \mathrm{BC}=0.01 \quad \mathrm{TRT} \times \mathrm{BC}=0.36$

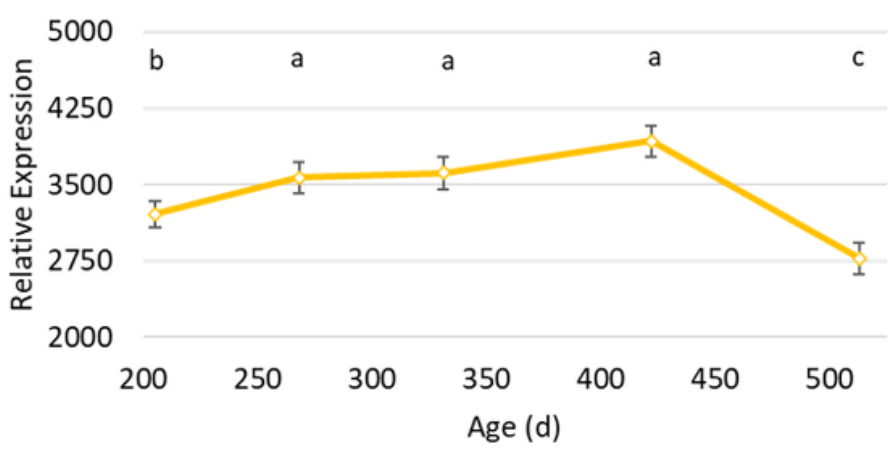

GPD1 BW

$\mathrm{TRT}=0.38 \quad \mathrm{BC}=0.01 \quad \mathrm{TRT} \times \mathrm{BC}=0.27$

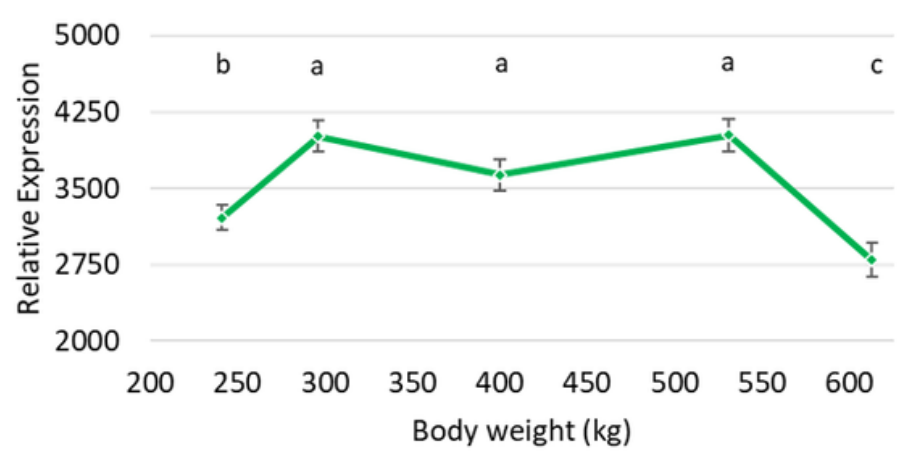

\section{Figure 19}

Expression of glycerol-3-phosphate dehydrogenase 1 (GPD1) in the longissimus muscle of steers compared A) at a similar number of days on feed (DOF) and age (205, 268, 331, 422, 513 days of age) or B) at a similar body weight (BW; 241, 296, 400, 531, $613 \mathrm{~kg}$ ). LSmean estimates across biopsy collections (BC) with different superscripts differ $(P \leq 0.05)$.

$20 \mathrm{~A}$

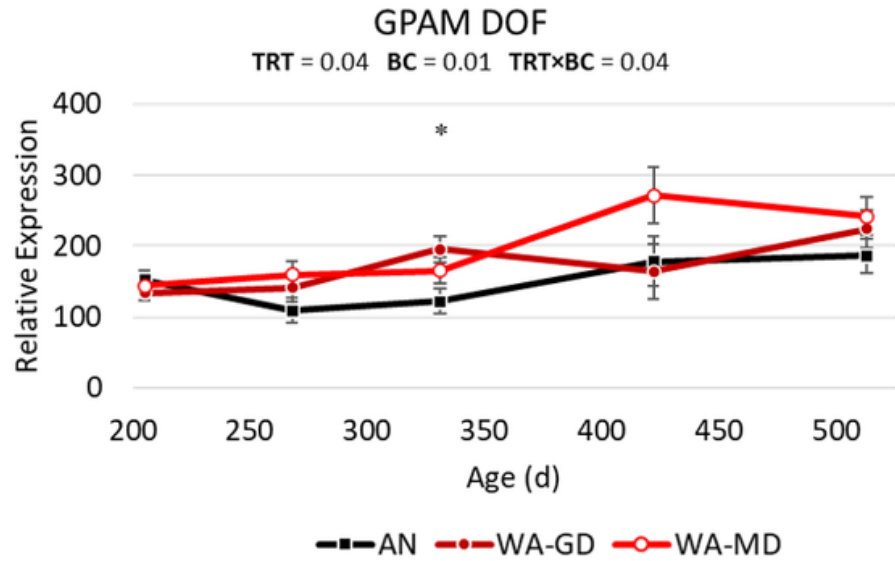

20B

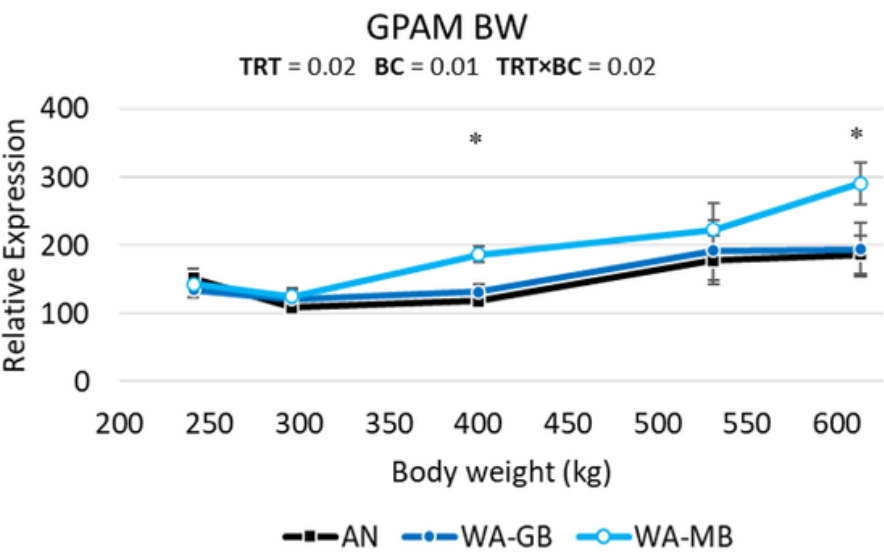

Figure 20

Expression of glycerol-3-phosphate acyltransferase (GPAM) in the longissimus muscle of steers compared A) at a similar number of days on feed (DOF) and age $(205,268,331,422,513$ days of age) or B) at a similar body weight (BW; 241, 296, 400, 531, $613 \mathrm{~kg}$ ). LSmean estimates between AN ( $\square$ ), WA-GD $(\bullet)$, and WA-MD $(\bullet)$ steers at a given biopsy with a $\left(^{*}\right)$ differ $(P \leq 0.05)$. LSmean estimates between AN $(\boldsymbol{\square})$, WA-GB $(\bullet)$, and WA-MB $(\bullet)$ steers at a given biopsy with a $\left({ }^{*}\right)$ differ $(\mathrm{P} \leq 0.05)$. 
AGPAT1 DOF

$\mathrm{TRT}=0.47 \quad \mathrm{BC}=0.02 \quad \mathrm{TRT} \times \mathrm{BC}=0.86$

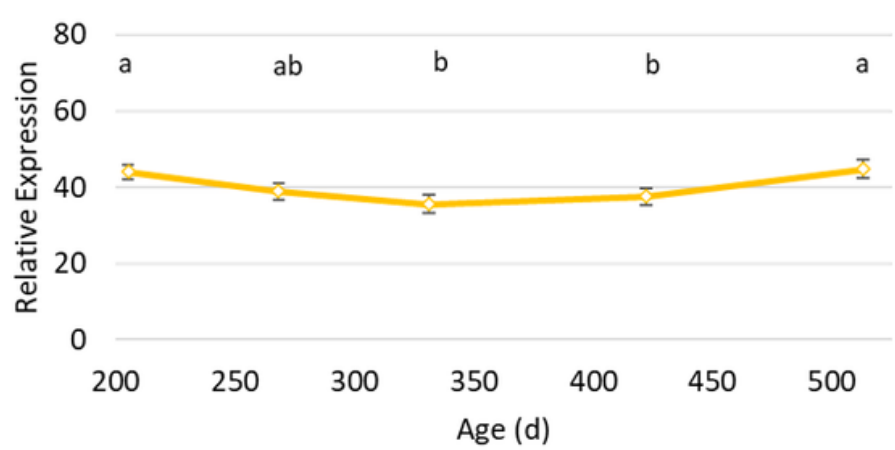

AGPAT1 BW

$\mathrm{TRT}=0.87 \quad \mathrm{BC}=0.02 \quad \mathrm{TRT} \times \mathrm{BC}=0.59$

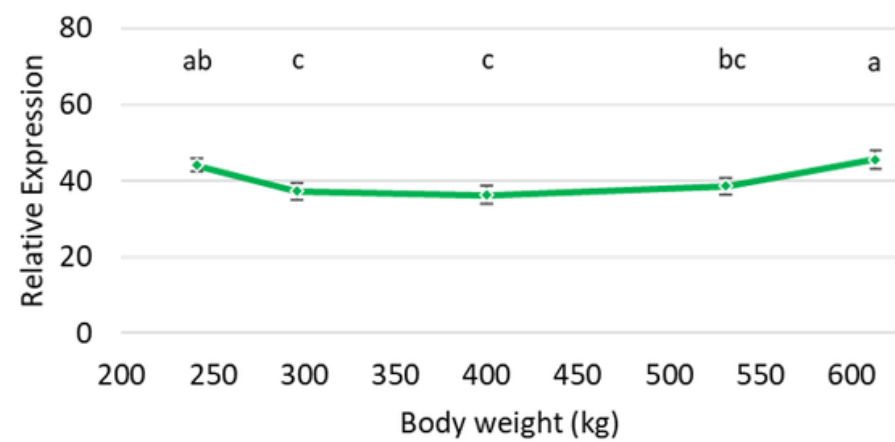

\section{Figure 21}

Expression of 1-acylglycerol-3-phosphate 0-acyltransferase 1 (AGPAT1) in the longissimus muscle of steers compared A) at a similar number of days on feed (DOF) and age $(205,268,331,422,513$ days of age) or B) at a similar body weight (BW; $241,296,400,531,613 \mathrm{~kg}$ ). LSmean estimates across biopsy collections $(\mathrm{BC})$ with different superscripts differ $(\mathrm{P} \leq 0.05)$.

22A

LPIN1 DOF

$\mathrm{TRT}=0.28 \quad \mathrm{BC}=0.01 \quad \mathrm{TRT} \times \mathrm{BC}=0.02$

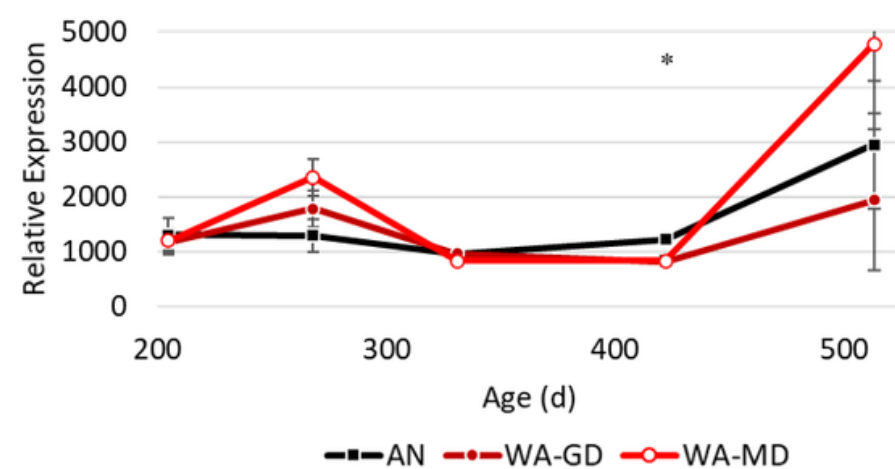

22B

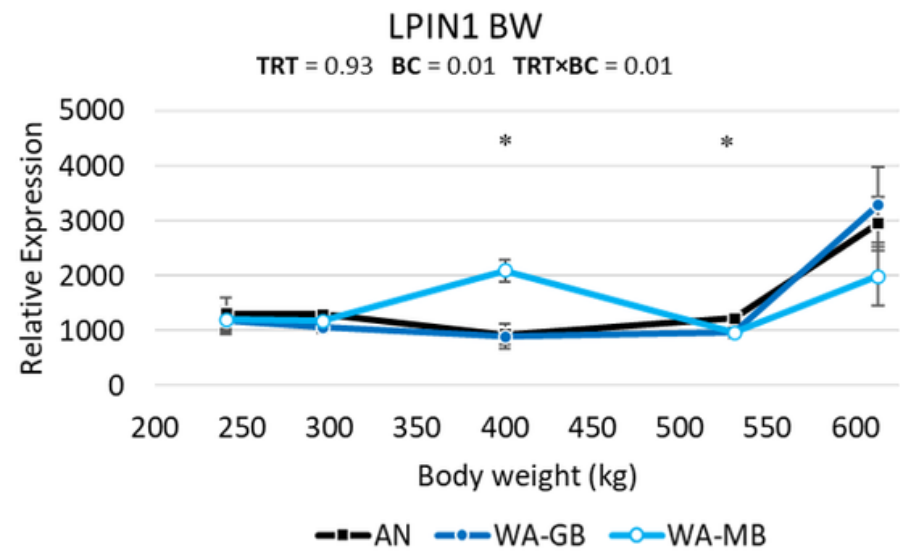

\section{Figure 22}

Expression of lipin 1 (LPIN1) in the longissimus muscle of steers compared A) at a similar number of days on feed (DOF) and age $(205,268,331,422,513$ days of age) or B) at a similar body weight (BW;

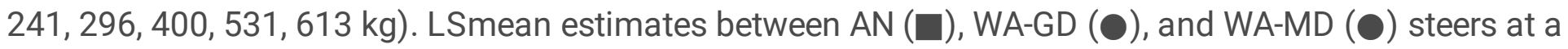
given biopsy with a $\left(^{*}\right)$ differ $(P \leq 0.05)$. LSmean estimates between AN $(\boldsymbol{\square})$, WA-GB $(\bullet)$, and WA-MB $(\bullet)$ steers at a given biopsy with a $\left(^{*}\right)$ differ $(P \leq 0.05)$. 


\section{DGAT1 DOF \\ TRT $=0.18 \quad B C=0.07 \quad$ TRT $\times B C=0.91$}

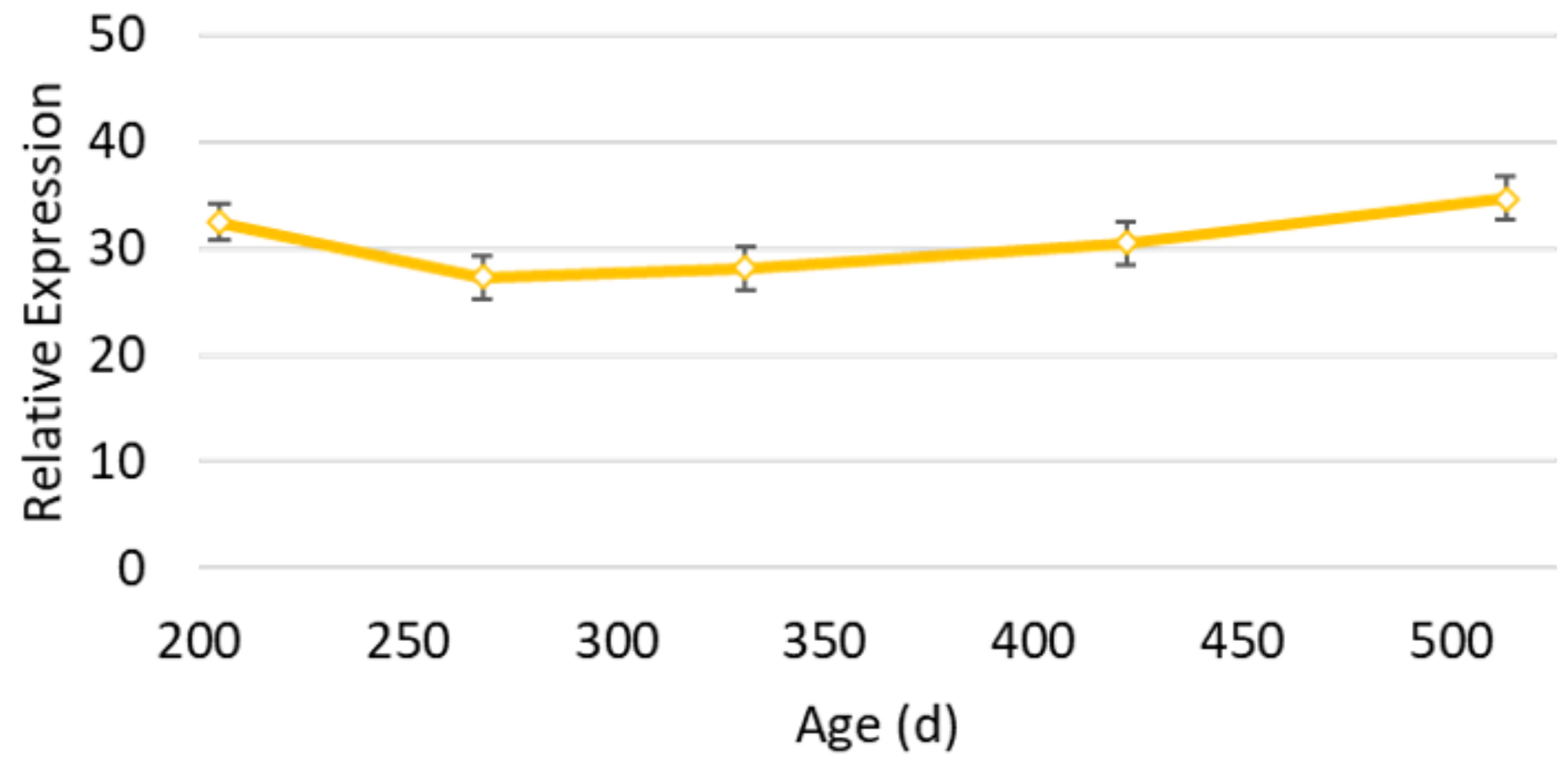

Figure 23

Expression of diacylglycerol 0-acyltransferase 1 (DGAT1) in the longissimus muscle of steers compared A) at a similar number of days on feed (DOF) and age $(205,268,331,422,513$ days of age) or B) at a similar body weight (BW; 241, 296, 400,531, $613 \mathrm{~kg}$ ). LSmean estimates across biopsy collections (BC) with different superscripts differ $(P \leq 0.05)$.

24A

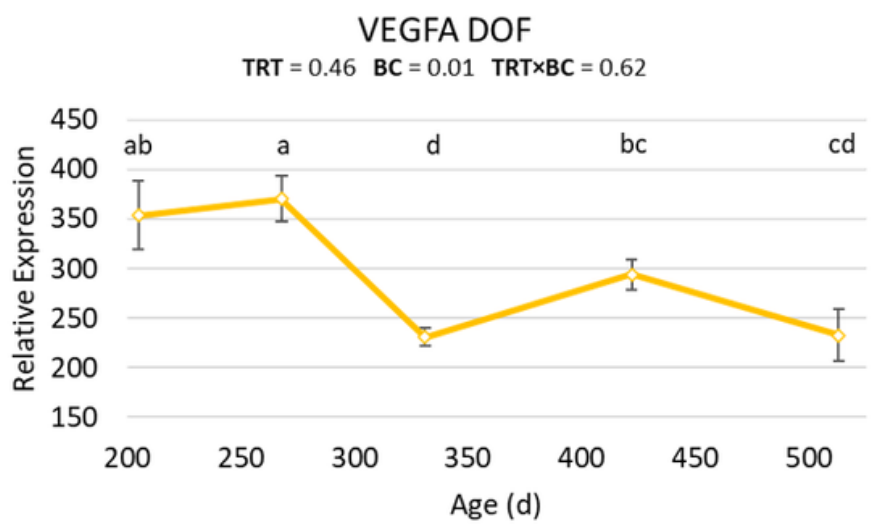

24B

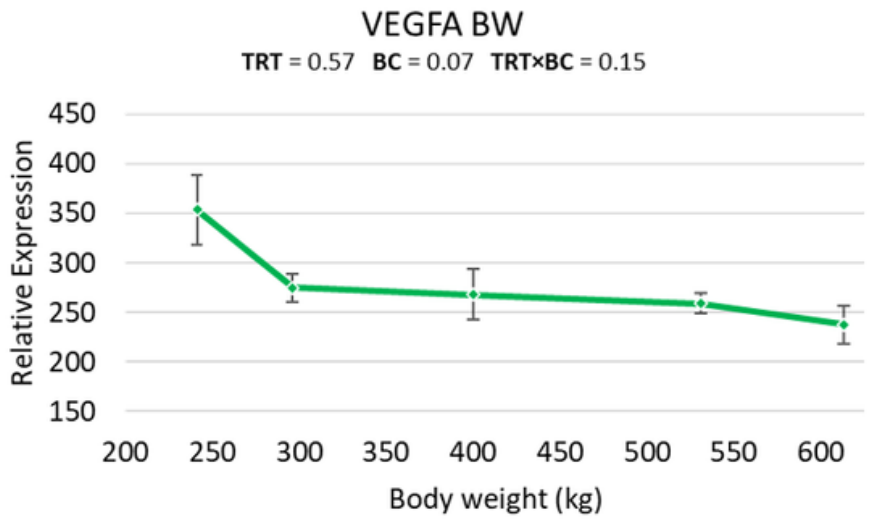

Figure 24

Expression of vascular endothelial growth factor A (VEGFA) in the longissimus muscle of steers compared A) at a similar number of days on feed (DOF) and age $(205,268,331,422,513$ days of age) or 
B) at a similar body weight (BW; $241,296,400,531,613 \mathrm{~kg}$ ). LSmean estimates across biopsy collections (BC) with different superscripts differ $(P \leq 0.05)$.

25A

ANGPT1 DOF

$T R T=0.61 \quad B C=0.04 \quad T R T \times B C=0.40$

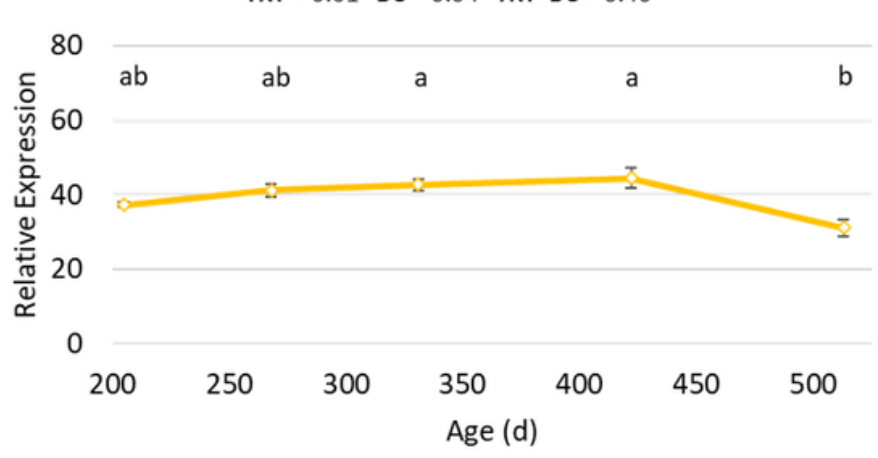

25B

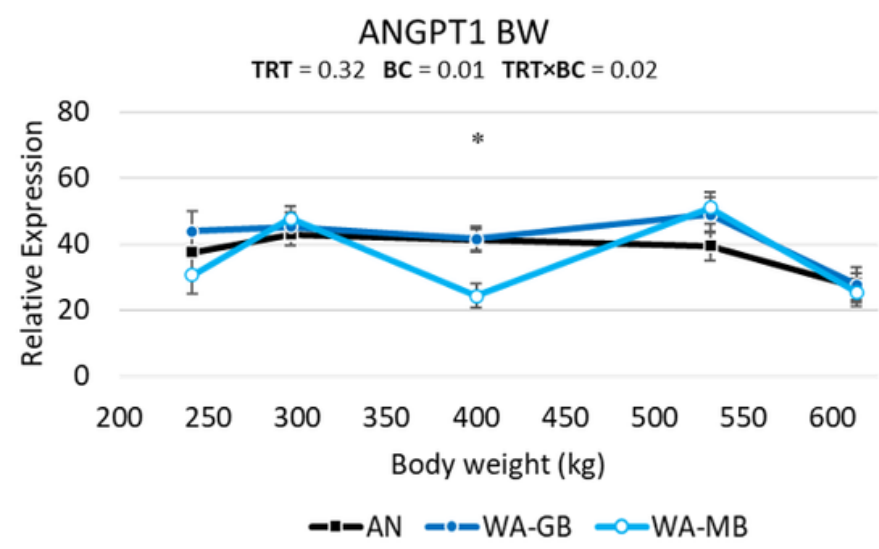

\section{Figure 25}

Expression of angiopoietin 1 (ANGPT1) in the longissimus muscle of steers compared A) at a similar number of days on feed (DOF) and age $(205,268,331,422,513$ days of age) or B) at a similar body weight (BW; 241, 296, 400, 531, $613 \mathrm{~kg}$ ). LSmean estimates across biopsy collections (BC) with different superscripts differ $(P \leq 0.05)$. LSmean estimates between AN $(\boldsymbol{\square})$, WA-GB $(\mathbf{\bullet})$, and WA-MB $(\mathbf{\bullet})$ steers at a given biopsy with a $\left(^{*}\right)$ differ $(P \leq 0.05)$.

26A

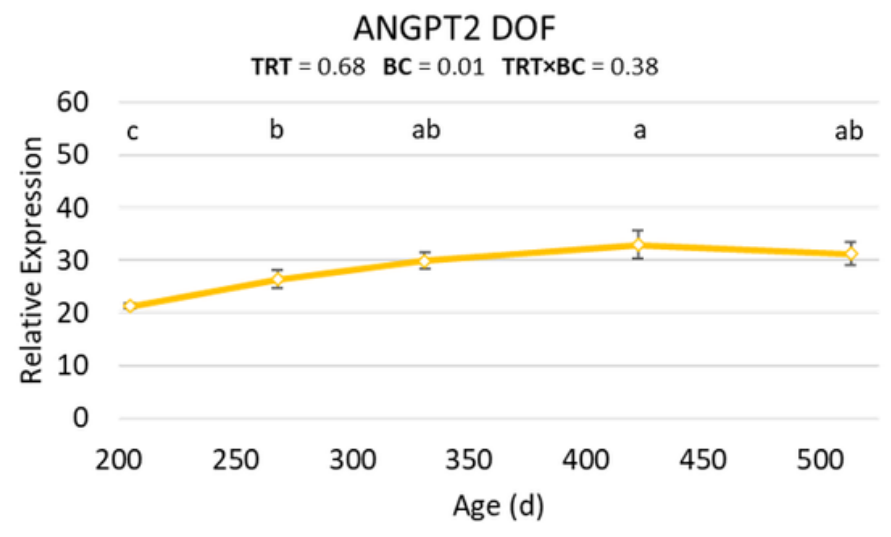

26B

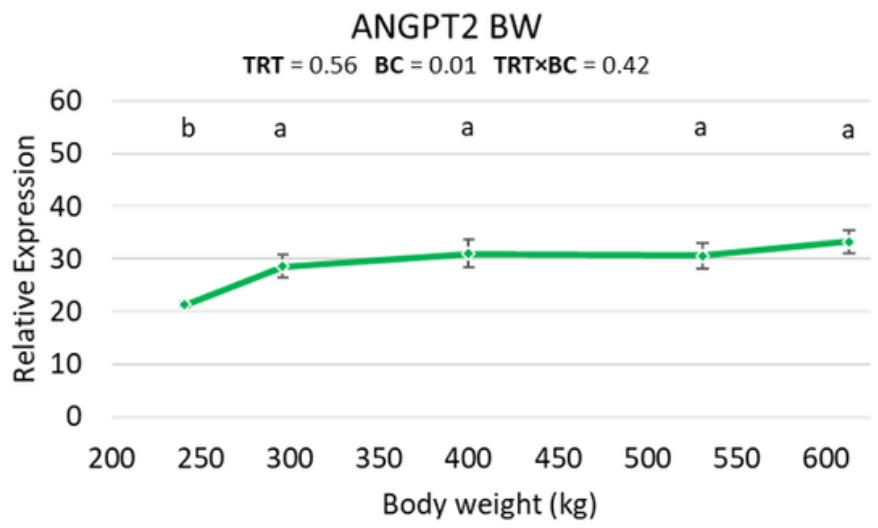

Figure 26

Expression of angiopoietin 2 (ANGPT2) in the longissimus muscle of steers compared A) at a similar number of days on feed (DOF) and age $(205,268,331,422,513$ days of age) or B) at a similar body weight (BW; 241, 296, 400, 531, $613 \mathrm{~kg}$ ). LSmean estimates across biopsy collections (BC) with different superscripts differ $(P \leq 0.05)$. 

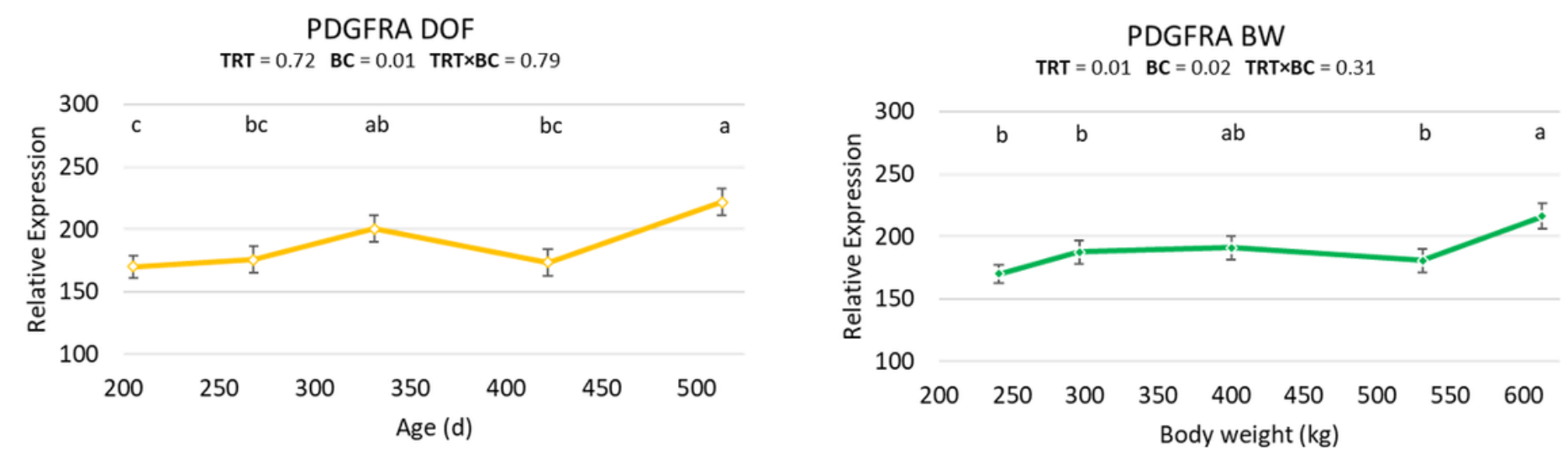

Figure 27

Expression of platelet derived growth factor alpha (PDGFA) in the longissimus muscle of steers compared A) at a similar number of days on feed (DOF) and age $(205,268,331,422,513$ days of age) or B) at a similar body weight (BW; 241, 296, 400, 531, $613 \mathrm{~kg}$ ). LSmean estimates across biopsy collections (BC) with different superscripts differ $(P \leq 0.05)$.

\section{PDGFRB DOF$$
\text { TRT }=0.33 \quad \text { BC }=0.01 \quad \text { TRT } \times B C=0.52
$$

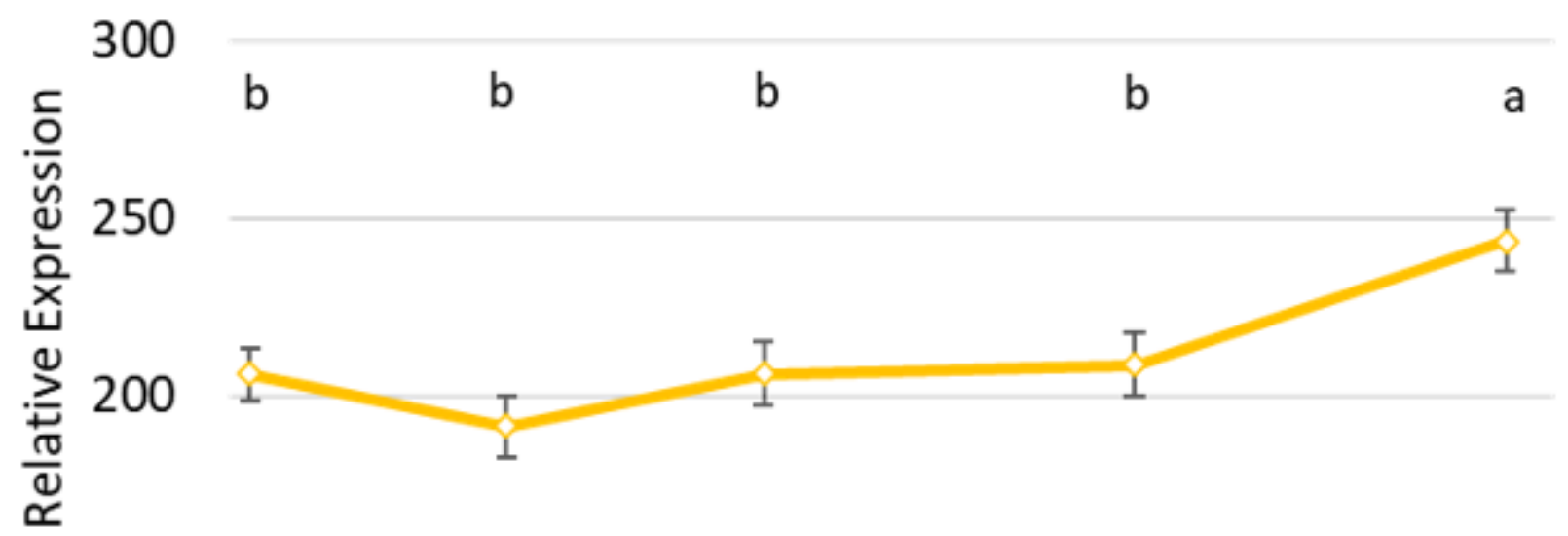

150

$\begin{array}{llllll}200 & 250 & 300 & 350 \quad 400 & 450 & 500 \\ & \text { Age (d) }\end{array}$

Figure 28

Expression of platelet derived growth factor beta (PDGFB) in the longissimus muscle of steers compared A) at a similar number of days on feed (DOF) and age $(205,268,331,422,513$ days of age) or B) at a similar body weight (BW; 241, 296, 400, 531, $613 \mathrm{~kg}$ ). LSmean estimates across biopsy collections (BC) with different superscripts differ $(P \leq 0.05)$. 

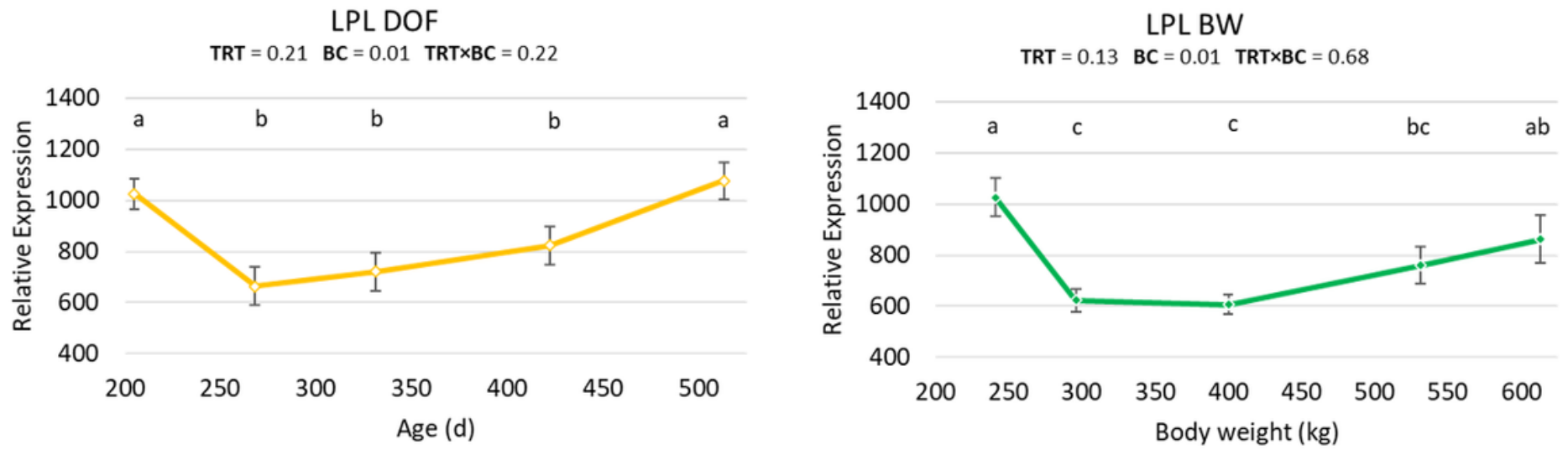

Figure 29

Expression of lipoprotein lipase ( $(\mathrm{PL})$ in the longissimus muscle of steers compared $A$ ) at a similar number of days on feed (DOF) and age $(205,268,331,422,513$ days of age) or B) at a similar body weight (BW; 241, 296, 400, 531, 613 kg). LSmean estimates across biopsy collections (BC) with different superscripts differ $(P \leq 0.05)$.

$30 \mathrm{~A}$

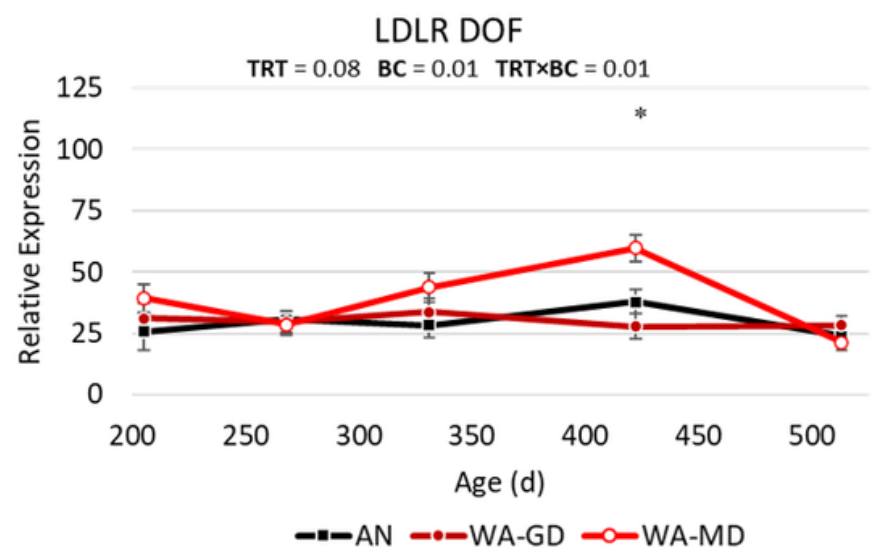

$30 \mathrm{~B}$

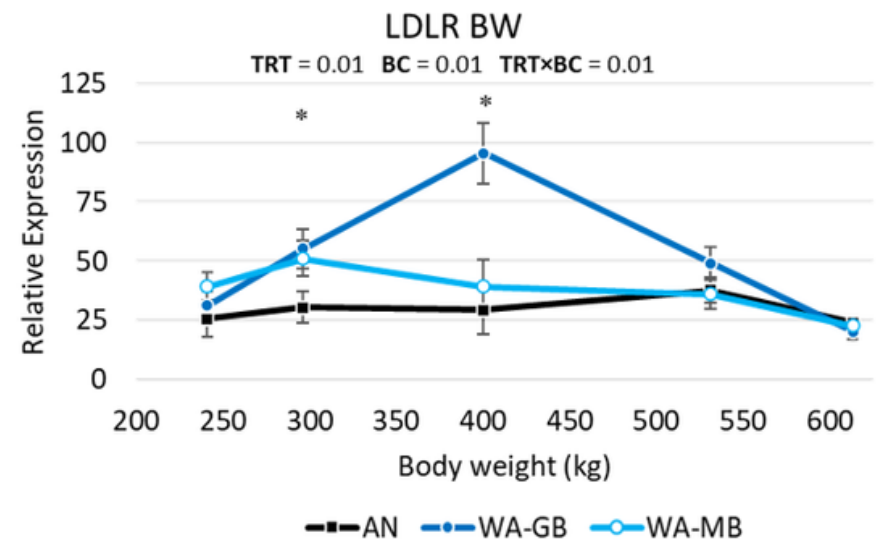

\section{Figure 30}

Expression of low density lipoprotein receptor (LDLR) in the longissimus muscle of steers compared $A$ ) at a similar number of days on feed (DOF) and age $(205,268,331,422,513$ days of age) or B) at a similar body weight (BW; 241, 296, 400, 531, $613 \mathrm{~kg}$ ). LSmean estimates between AN (ם), WA-GD (0), and WAMD (๑) steers at a given biopsy with a $\left(^{*}\right)$ differ $(P \leq 0.05)$. LSmean estimates between AN $(\square)$, WA-GB $(\bullet)$, and WA-MB $(\bullet)$ steers at a given biopsy with a $\left(^{*}\right)$ differ $(P \leq 0.05)$. 

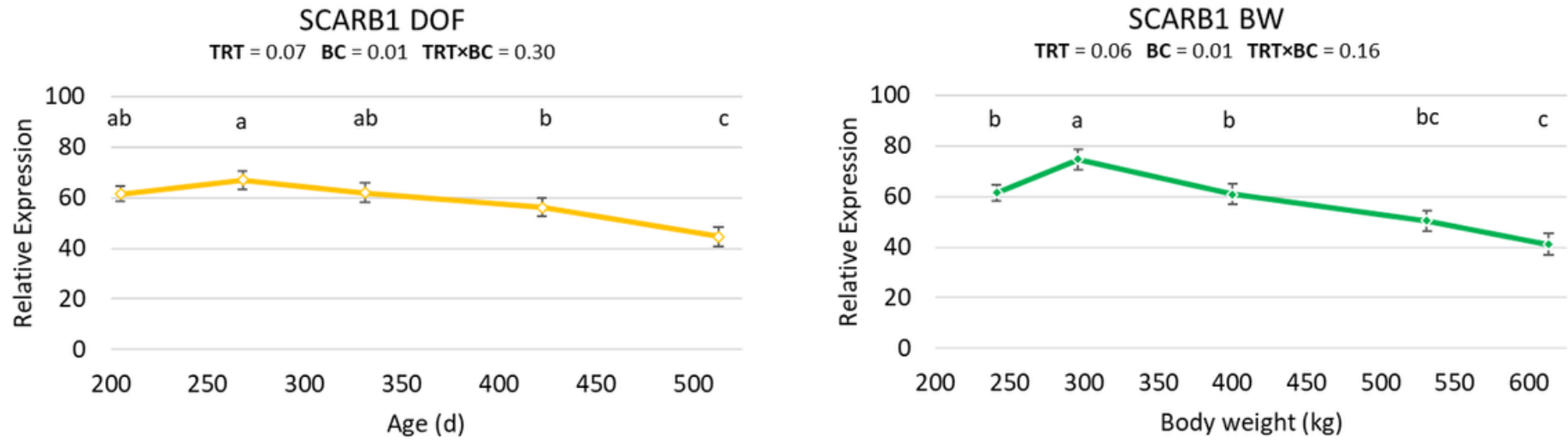

\section{Figure 31}

Expression of scavenger receptor class B member1 (SCARB1) in the longissimus muscle of steers compared A) at a similar number of days on feed (DOF) and age $(205,268,331,422,513$ days of age) or B) at a similar body weight (BW; 241, 296, 400, 531, $613 \mathrm{~kg}$ ). LSmean estimates across biopsy collections (BC) with different superscripts differ $(P \leq 0.05)$.

$32 \mathrm{~A}$

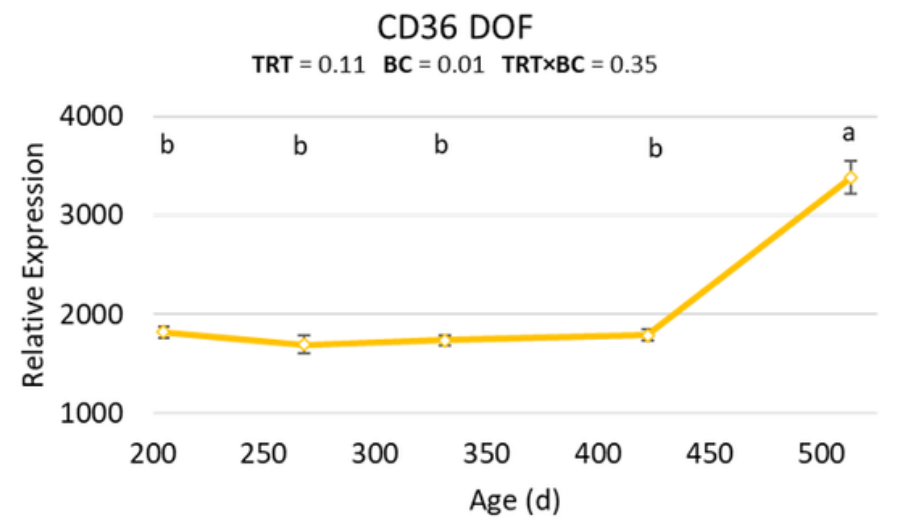

32B

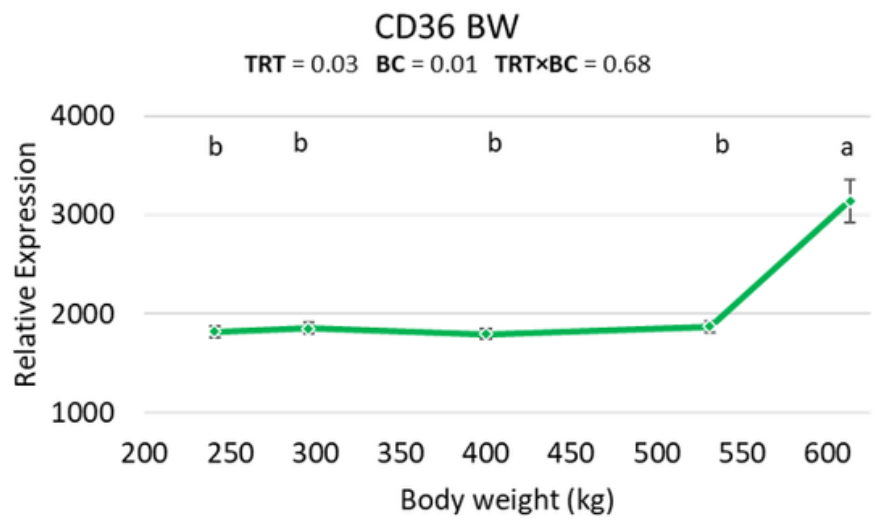

\section{Figure 32}

Expression of cd36 molecule (CD36) in the longissimus muscle of steers compared A) at a similar number of days on feed (DOF) and age $(205,268,331,422,513$ days of age) or B) at a similar body weight (BW; 241, 296, 400, 531, 613 kg). LSmean estimates across biopsy collections (BC) with different superscripts differ $(P \leq 0.05)$. 
FATP1 DOF

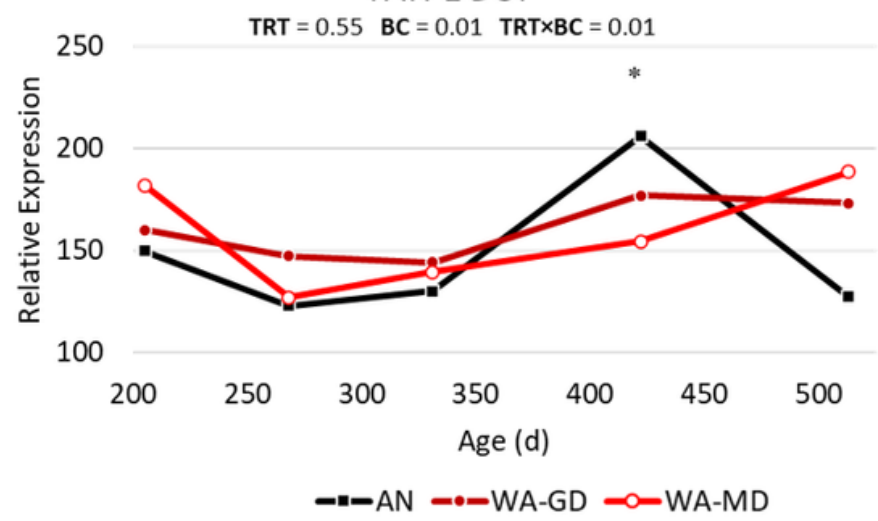

FATP1 BW

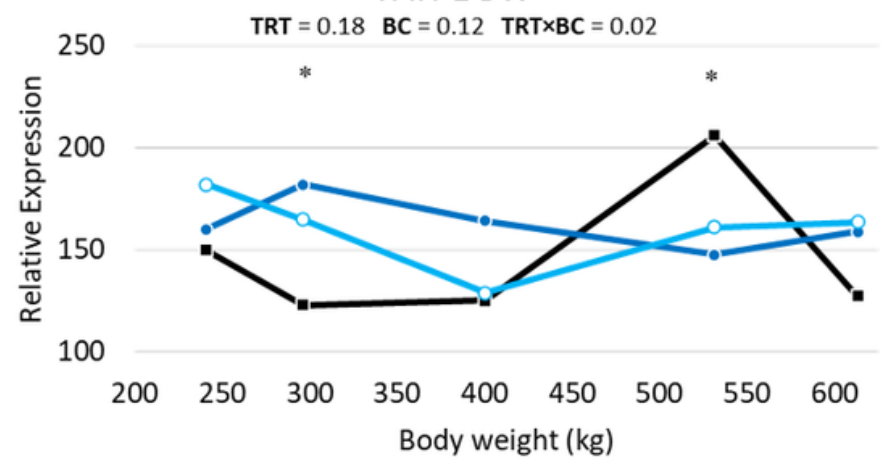

--AN $-\bullet-W A-G B \quad \longrightarrow$ WA-MB

\section{Figure 33}

Expression of fatty acid transport protein 1 (FATP1) in the longissimus muscle of steers compared A) at a similar number of days on feed (DOF) and age $(205,268,331,422,513$ days of age) or B) at a similar body weight (BW; 241, 296, 400, 531, $613 \mathrm{~kg}$ ). LSmean estimates between AN ( $\boldsymbol{\square})$, WA-GD ( $(\mathbf{)})$, and WAMD (๑) steers at a given biopsy with a $\left(^{*}\right)$ differ $(P \leq 0.05)$. LSmean estimates between AN $(\square)$, WA-GB $(\bullet)$, and WA-MB $(\bullet)$ steers at a given biopsy with a $(*)$ differ $(P \leq 0.05)$.

34A

FABP4 DOF

$\mathrm{TRT}=0.07 \quad \mathrm{BC}=0.04 \quad \mathrm{TRT} \times \mathrm{BC}=0.43$

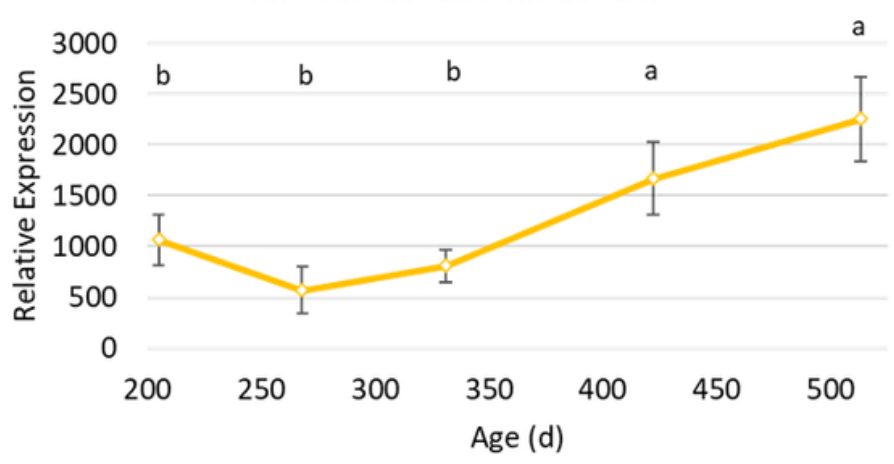

34B

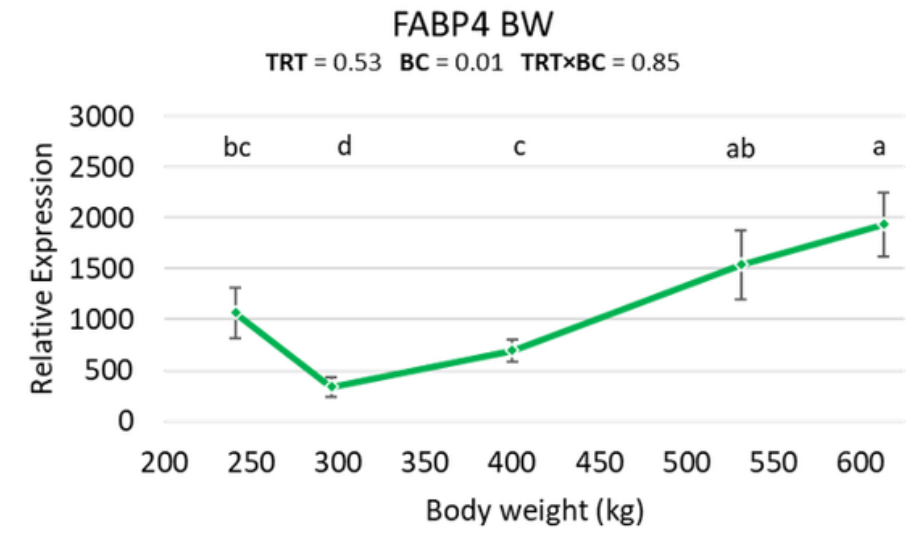

\section{Figure 34}

Expression of fatty acid binding protein 4 (FABP4) in the longissimus muscle of steers compared A) at a similar number of days on feed (DOF) and age $(205,268,331,422,513$ days of age) or B) at a similar body weight (BW; 241, 296, 400, 531, $613 \mathrm{~kg}$ ). LSmean estimates across biopsy collections (BC) with different superscripts differ $(P \leq 0.05)$. 

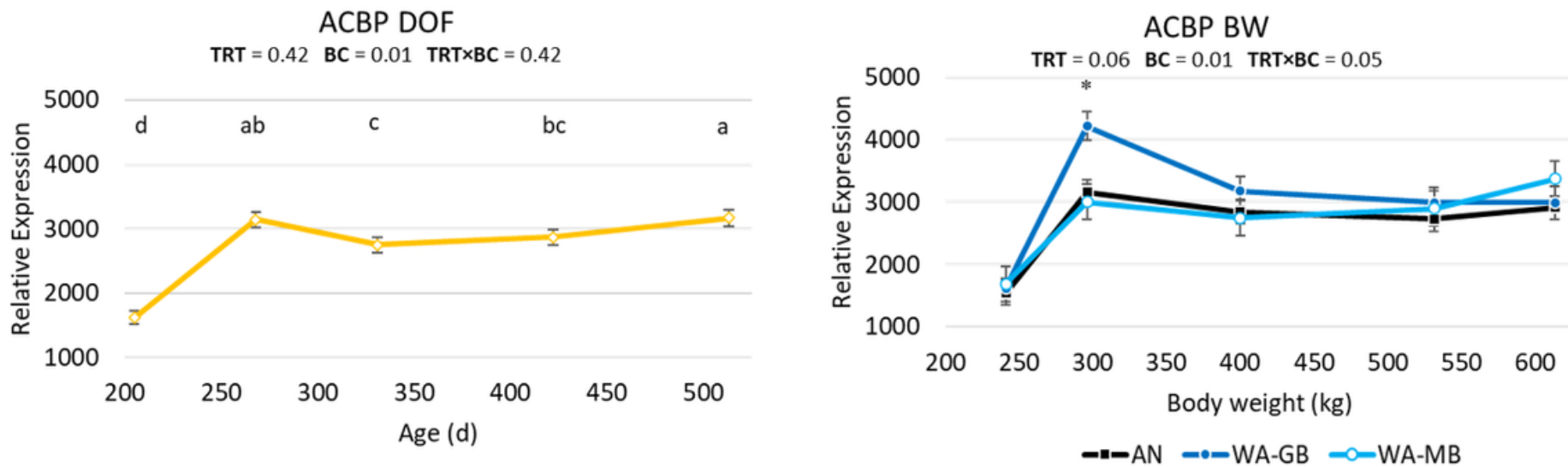

Figure 35

Expression of acyl-CoA binding protein (ACBP) in the longissimus muscle of steers compared $A$ ) at a similar number of days on feed (DOF) and age $(205,268,331,422,513$ days of age) or B) at a similar body weight (BW; 241, 296, 400, 531, $613 \mathrm{~kg}$ ). LSmean estimates across biopsy collections (BC) with different superscripts differ $(P \leq 0.05)$. LSmean estimates between AN $(\boldsymbol{\square})$, WA-GB $(\mathbf{\bullet})$, and WA-MB ( $(\bullet)$ steers at a given biopsy with a $\left(^{*}\right)$ differ $(P \leq 0.05)$.

$36 \mathrm{~A}$

PPARA DOF

TRT $=0.53 \quad B C=0.08 \quad$ TRT $\times$ BC $=0.97$

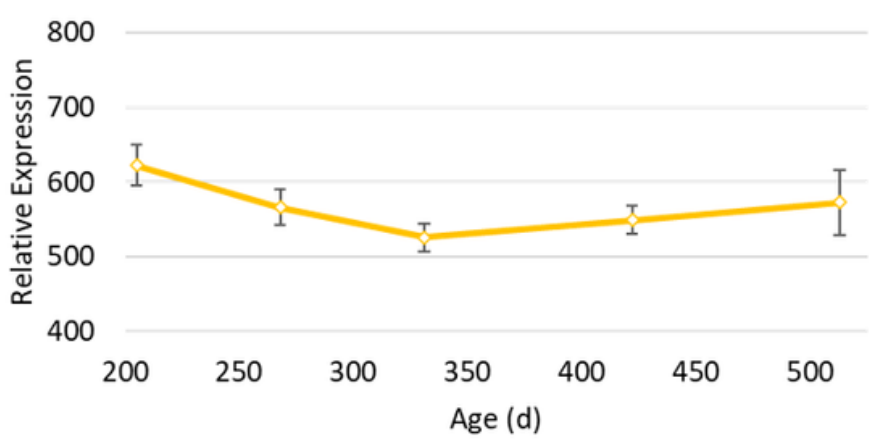

36B

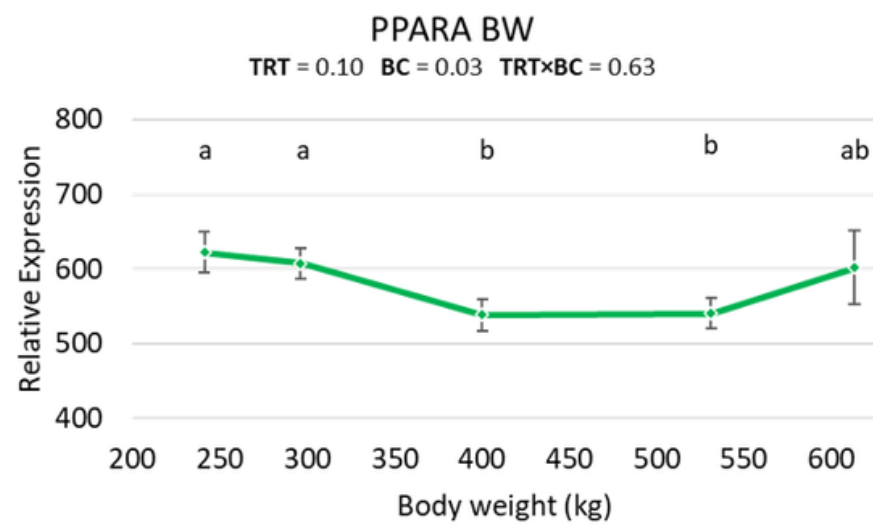

\section{Figure 36}

Expression of peroxisome proliferator activated receptor alpha (PPARA) in the longissimus muscle of steers compared A) at a similar number of days on feed (DOF) and age $(205,268,331,422,513$ days of age) or B) at a similar body weight (BW; $241,296,400,531,613 \mathrm{~kg}$ ). LSmean estimates across biopsy collections $(\mathrm{BC})$ with different superscripts differ $(\mathrm{P} \leq 0.05)$. 

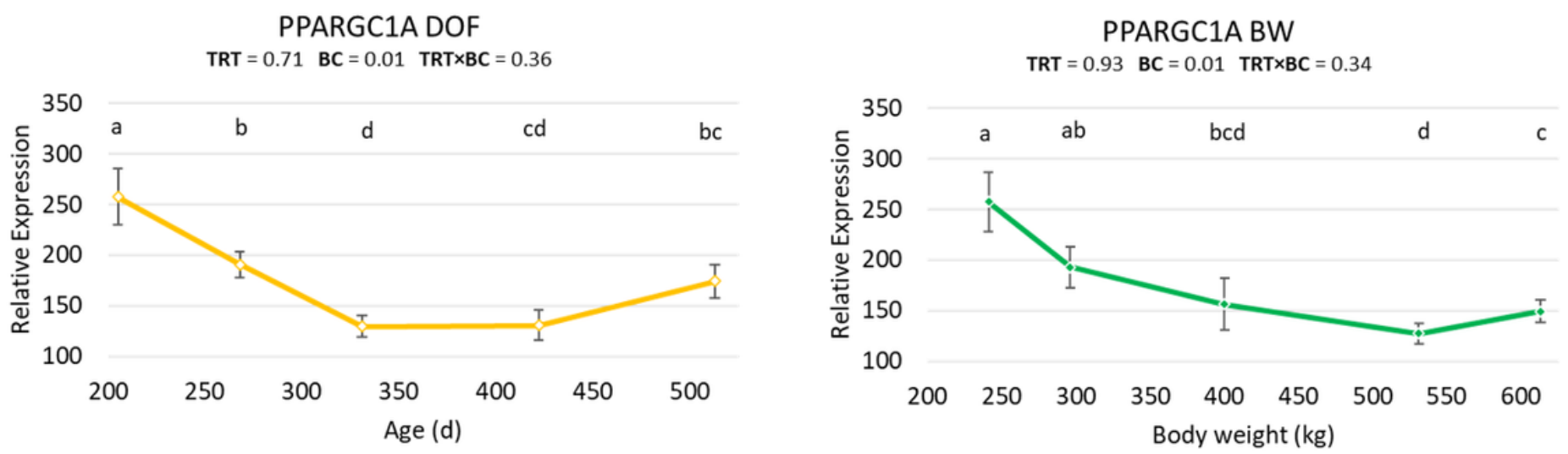

\section{Figure 37}

Expression of peroxisome proliferator activated receptor coactivator 1 alpha (PPARGC1A) in the longissimus muscle of steers compared $A)$ at a similar number of days on feed (DOF) and age $(205,268$, $331,422,513$ days of age) or B) at a similar body weight (BW; 241, 296, 400, 531, $613 \mathrm{~kg}$ ). LSmean estimates across biopsy collections (BC) with different superscripts differ $(P \leq 0.05)$.

38A

ATGL DOF

$\mathrm{TRT}=0.72 \quad \mathrm{BC}=0.01 \quad \mathrm{TRT} \times \mathrm{BC}=0.71$

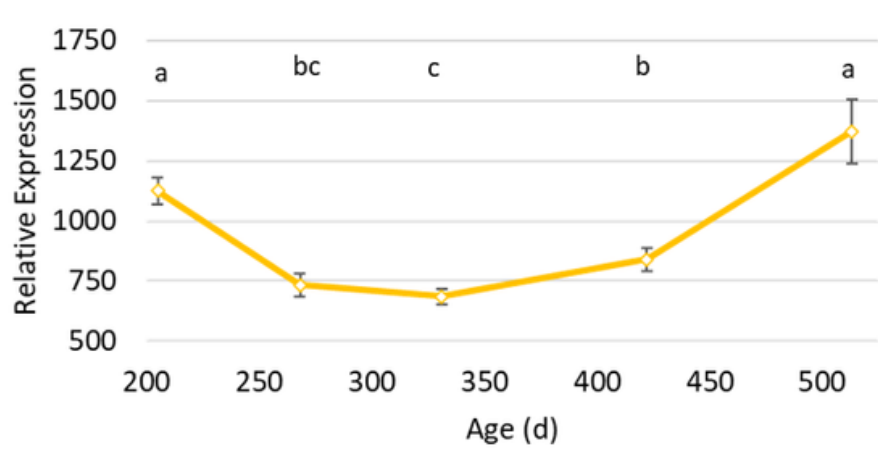

38B

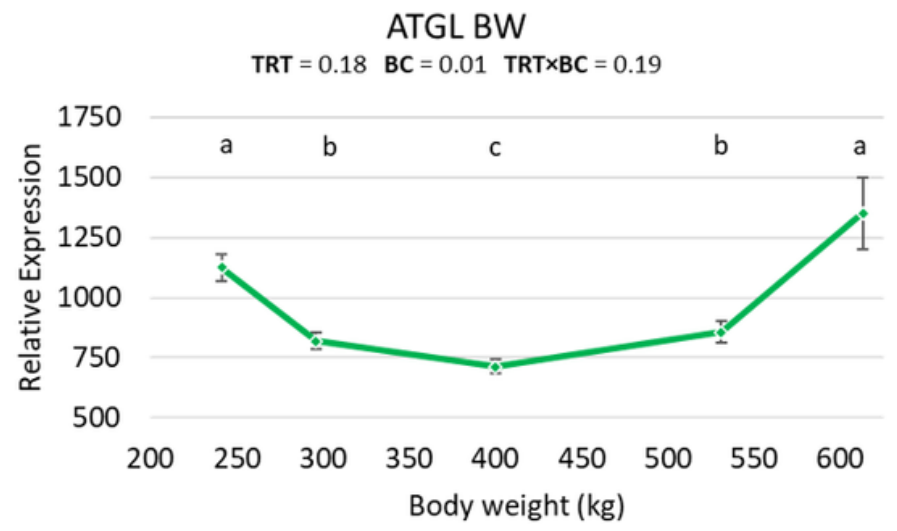

Figure 38

Expression of adipose triglyceride lipase (ATGL) in the longissimus muscle of steers compared A) at a similar number of days on feed (DOF) and age $(205,268,331,422,513$ days of age) or B) at a similar body weight (BW; 241, 296, 400, 531, $613 \mathrm{~kg}$ ). LSmean estimates across biopsy collections (BC) with different superscripts differ $(P \leq 0.05)$. 
HSL DOF

$T R T=0.04 \quad B C=0.01 \quad T R T \times B C=0.47$

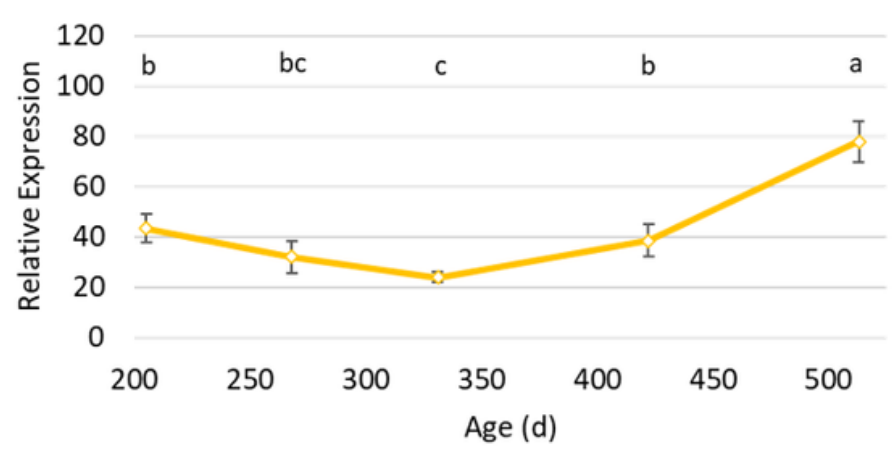

HSL BW

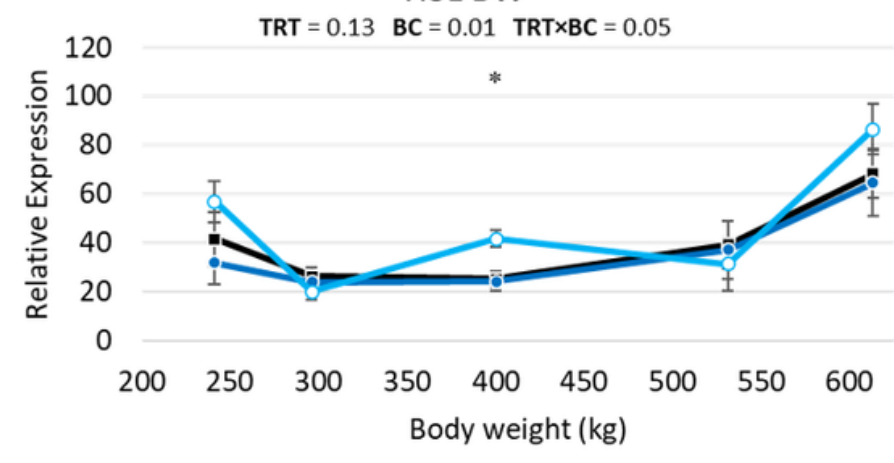

$\because-A N \stackrel{-W A-G B}{\longrightarrow} \longrightarrow$ WA-MB

\section{Figure 39}

Expression of hormone sensitive lipase (HSL) in the longissimus muscle of steers compared A) at a similar number of days on feed (DOF) and age $(205,268,331,422,513$ days of age) or B) at a similar body weight (BW; 241, 296, 400, 531, $613 \mathrm{~kg}$ ). LSmean estimates across biopsy collections (BC) with different superscripts differ $(P \leq 0.05)$. LSmean estimates between AN $(\boldsymbol{\square})$, WA-GB $(\bullet)$, and WA-MB ( $(\bullet)$ steers at a given biopsy with a $\left(^{*}\right)$ differ $(P \leq 0.05)$.

$40 \mathrm{~A}$

MGL DOF

$\mathrm{TRT}=0.51 \quad \mathrm{BC}=0.01 \quad \mathrm{TRT} \times \mathrm{BC}=0.10$

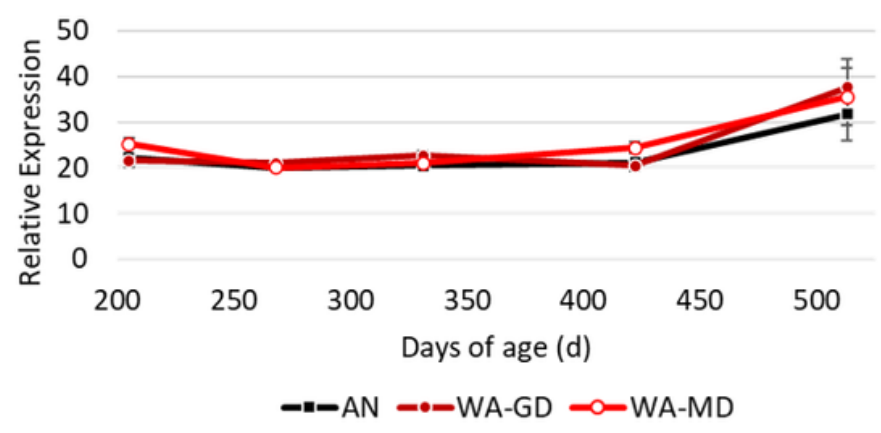

$40 B$

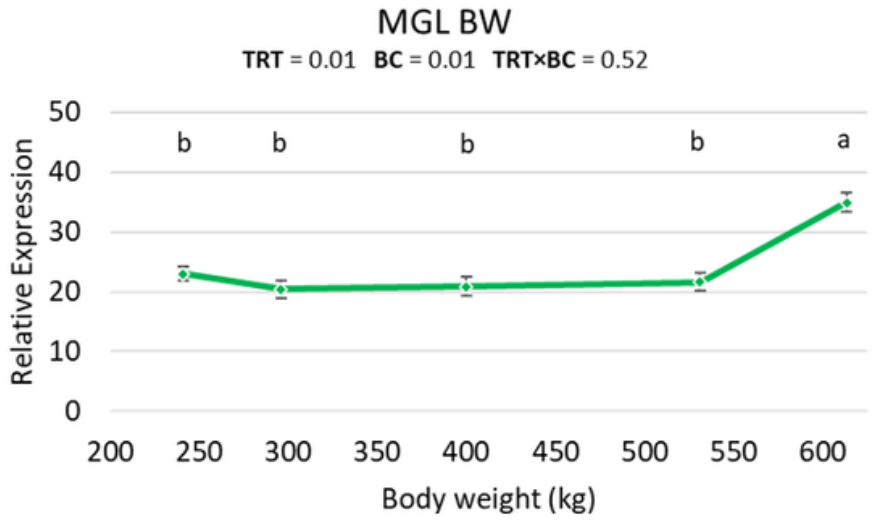

\section{Figure 40}

Expression of monoglyceride lipase (MGL) in the longissimus muscle of steers compared A) at a similar number of days on feed (DOF) and age $(205,268,331,422,513$ days of age) or B) at a similar body weight (BW; 241, 296, 400, 531, $613 \mathrm{~kg}$ ). LSmean estimates across biopsy collections (BC) with different superscripts differ (P $\leq 0.05$ LSmean estimates between AN $(\boldsymbol{\square})$, WA-GD $(\bullet)$, and WA-MD $(\bullet)$ steers at a given biopsy with a $\left.{ }^{*}\right)$ differ $(P \leq 0.05)$. 

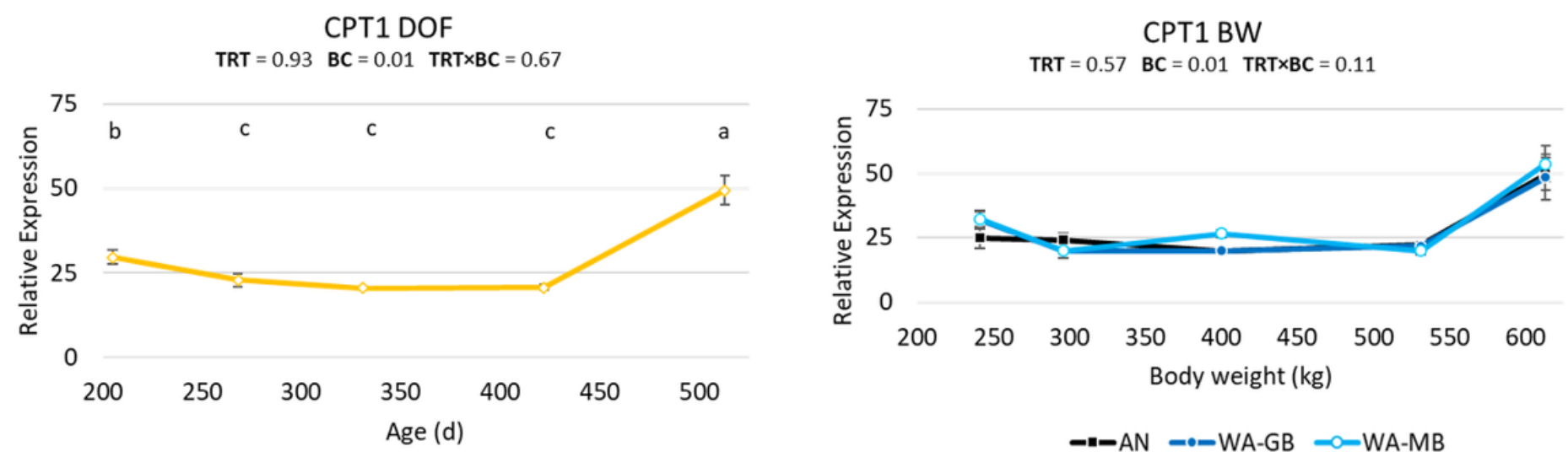

\section{Figure 41}

Expression of carnitine palmitoyltransferases 1 (CPT1) in the longissimus muscle of steers compared A) at a similar number of days on feed (DOF) and age $(205,268,331,422,513$ days of age) or B) at a similar body weight (BW; 241, 296, 400, 531, $613 \mathrm{~kg}$ ). LSmean estimates across biopsy collections (BC) with different superscripts differ $(P \leq 0.05)$. LSmean estimates between AN $(\boldsymbol{\square})$, WA-GB $(\boldsymbol{\bullet})$, and WA-MB $(\circlearrowleft)$ steers at a given biopsy with a $(*)$ differ $(P \leq 0.05)$.

$42 \mathrm{~A}$

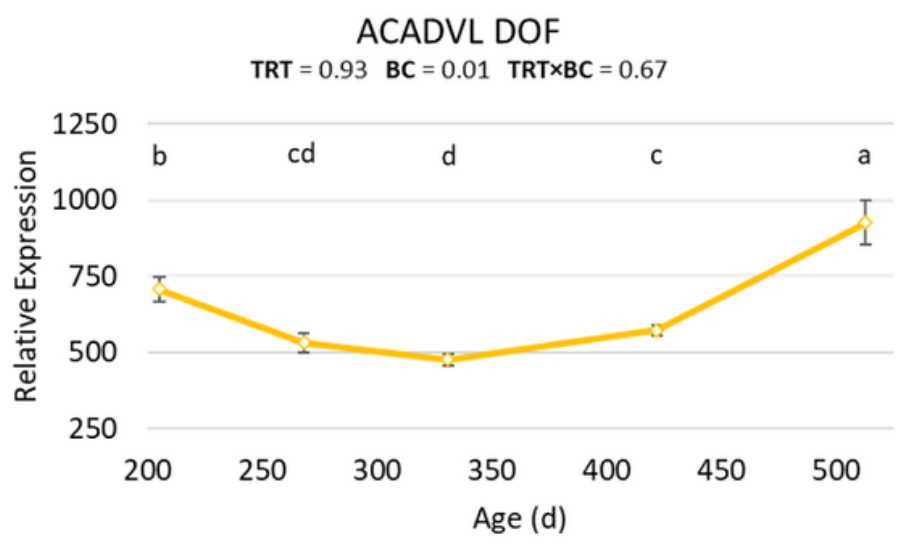

$42 B$

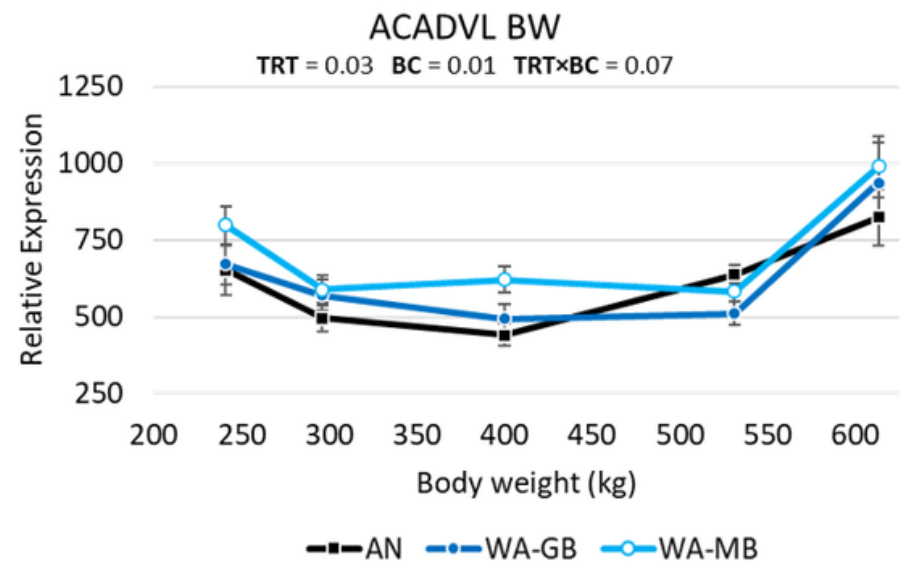

\section{Figure 42}

Expression of very long chain acyl-CoA dehydrogenase (ACADVL) in the longissimus muscle of steers compared A) at a similar number of days on feed (DOF) and age $(205,268,331,422,513$ days of age) or B) at a similar body weight (BW; 241, 296, 400, 531, $613 \mathrm{~kg}$ ). LSmean estimates across biopsy collections (BC) with different superscripts differ $(P \leq 0.05)$. LSmean estimates between AN ( $\boldsymbol{\square})$, WA-GB $(\mathbf{\bullet})$, and WA-MB (๑) steers at a given biopsy with a $(*)$ differ $(P \leq 0.05)$. 